\title{
Sponsors of SENS6
}

\section{international}

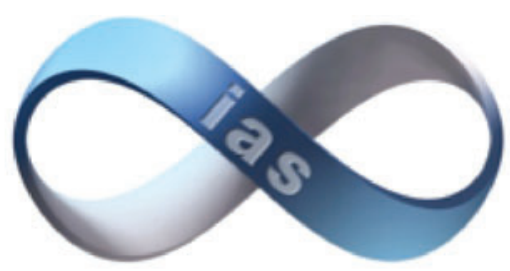

antiaging-systems.com

LifeExtension

入 Life Length

I\&A IMMUNITY \& AGEING

Longevity \&
Healthspan

Kay 


\section{Meeting Program}

\section{TUESDAY SEPTEMBER 3RD}

\section{Session 1: Calorie restriction in primates}

16:15 Donald Ingram

Pennington Biomedical Research

Center, USA

16:35 Rozalyn Anderson

University of Wisconsin, USA

16:55 Donald Ingram/Rozalyn Anderson

Pennington Biomedical Research Center, USA/University of Wisconsin, USA

17:10 Donald Ingram/Rozalyn Anderson

Pennington Biomedical Research Center, USA/University of Wisconsin, USA

17:30 Luigi Fontana

Washington University, USA/Salerno

University, Italy

18:00 Coffee
Monkeying around with calorie restriction: is a calorie a calorie?

Impact of CR on aging in rhesus monkeys - a focus on metabolism

Outcomes from parallel CR monkey studies at NIA and UW Madison

Q\&A Session

Will calorie restriction work in humans?

\section{Session 2: Small-molecule interventions effective at late age}

18:30 Dongsheng Cai

Albert Einstein College of Medicine, USA

19:00 Danica Chen

University of California at Berkeley, USA

19:30 Frank Madeo

University of Graz, Austria

20:00 Arie Budovsky

Ben-Gurion University of the Negev, Israel

20:15 Dinner

21:15 Poster Session
Hypothalamic control of aging and longevity

Sirtuin regulation of metabolism and stem cells

Spermidine induces autophagy in multiple species and mimics calorie restriction

Screening of medicinal plants in the Judea region (Israel) for geroprotective activities 


\section{WEDNESDAY SEPTEMBER 4TH}

\section{Session 3: Telomeres and cancer}

08:30 Zhenyu Ju

Hangzhou Normal University, China

09:00 Haroldo Silva

SENS Research Foundation, USA

09:30 Rigdon Lentz

Georgetown University, USA

10:00 Coffee
Telomere dysfunction and stem cell aging

ALTered cancer cells: Uncovering the genetic basis of ALT (Alternative Lengthening of Telomeres)

Apheresis of TNF and IL-2 disrupts tumour immunoevasion

\section{Session 4: Combating persistent viruses}

10:30 Charles Cao

University of Florida, USA

11:00 Paul Lehner

Cambridge University, UK

11:30 Todd Rider

Massachusetts Institute

of Technology, USA

12:00 Lunch
Nanoparticle-based artificial RNA silencing machinery for antiviral therapy

Latency-associated degradation of the MRP1 drug transporter presents a novel therapeutic target for latent human cytomegalovirus infection

PANACEA broad-spectrum antiviral therapeutics

\section{Session 5: Short talks, cell senescence and anergy}

13:15 Gregory Chin

SENS Research Foundation, USA

13:30 Lia Ginaldi

University of L'Aquila, Italy

13:45 Alexandra Stolzing

Fraunhofer Institute for Cell Therapy

and Immunology, Germany

14:00 John Sedivy

Brown University, USA

14:30 Kevin Perrott

Buck Institute for Aging Research, USA

15:00 Janko Nikolich-Zugich

University of Arizona, USA

15:30 Jan van Deursen

Mayo Clinic, USA

16:00 Coffee
SRF Education: Training Future SENS Researchers

Effect of senescence on bone remodelling: the role of inflammageing

Transplantation of in vitro derived microglia

Chromatin and epigenetic dynamics in senescence phenotypes

Targeting the senescence-associated secretory

phenotype

Life-long persistent viral infection alters the naïve

T-cell pool, impairing CD8 T-cell immunity in late life

Stem and progenitor cell senescence in aging 


\section{Session 6: Disruptive medicine}

16:30 Alan Russell

Carnegie Mellon University, USA

17:00 Richard Barker

CASMI, UK

17:30 Sam Parnia

SUNY Stony Brook, USA

18:00 Coffee
The disruptive potential of tissue engineering

Accelerating translational research processes from bench to clinic

Redefining and reversing death: what has resuscitation science taught us about death and what happens when we die?

\section{Session 7: Cardiovascular aging}

18:30 Elizabeth Corder

Matrix Genomics, Inc., USA

19:00 David Spiegel

Yale University, USA

19:30 Brian $\mathbf{O}^{\prime}$ Nuallain

Harvard University, USA

20:00 Dinner

21:00 Poster session
Low risk for coronary artery disease and findings in the TERC region on $3 q 26$

Chemical and biological approaches to understanding advanced glycation end-products (AGEs)

Innate and vaccine-generated antibodies against transthyretin amyloids

\section{THURSDAY SEPTEMBER 5TH}

\section{Session 8: Beyond organ transplantation}

08:30 John Jackson

Wake Forest Institute for Regenerative

Medicine, USA

09:00 Eric Lagasse

McGowan Institute for Regenerative

Medicine, USA

09:30 Steve Van Sickle

Arigos Inc.

10:00 Coffee
Characterization of decellularized thymus scaffolds

for use in engineering thymus tissue

Ectopic organogenesis in lymph node

Improved technology for organ cryopreservation by vitrification

\section{Session 9: Cellular regeneration}

10:30 Robin Franklin

Cambridge University, UK
Myelin regeneration and ageing 
11:00 Graca Almeida-Porada

Wake Forest Institute for Regenerative

Medicine, USA

11:30 Malcolm Maden

University of Florida, USA

12:00 Lunch
Engineering stem cells for liver and gut regeneration

Regeneration in the adult organism - a cure for ageing?

\section{Session 10: SENS Lecture and short talks}

13:30 George Church

Harvard University, USA

14:30 Justin Rebo

SENS Research Foundation, USA

14:45 James Larrick

Panorama Research Institute, USA

15:00 João de Magalhães

University of Liverpool, UK

15:15 Jean Hébert

Albert Einstein College of

Medicine, USA

15:30 John Furber

Legendary Pharmaceuticals, USA

15:45 Axel Kowald

University of Newcastle, UK

16:00 Coffee
SENS Lecture: Technologies for Reading, Writing \& Interpreting Omes

Treating Cytopenia with ESC Derived Blood Cells

Sodium-Potassium ATPase modulation for Aging Related Disease

Sequencing the genome of the longest-lived mammal to identify longevity assurance mechanisms

What good is an old brain in a young body - a strategy for regenerating the neocortex

Inducing exocytosis to remove lipofuscin

Is transcription the key to the selection advantage of mitochondrial deletion mutants?

\section{Session 11: Mitochondria}

16:30 Richard Youle

National Institute for Neurological

Disorders and Stroke, USA

17:00 Rafal Smigrodzki

Gencia Corporation, USA

17:30 Matthew O'Connor

SENS Research Foundation, USA
Mitochondrial quality control: links to Parkinson's disease and aging models

Targeted mitochondrial therapeutics in aging

Rescue of mtDNA mutations by allotopic expression of engineered mitochondrial genes

18:00 Coffee

\section{Session 12: Advances in gene delivery}

18:30 Matthew Scholz

Immusoft Corporation
Utilizing B cells to recreate the biochemical environment of youth 
19:00 Yanru Chen-Tsai

Stanford University, USA

19:30 Dirk Hockemeyer

University of California at Berkeley, USA
Phage integrase for targeted gene therapy in mouse models

Molecular mechanisms of telomerase regulation in genetically defined human stem cell models

20:00 Dinner

21:00 Poster Session

\section{FRIDAY SEPTEMBER 6TH}

\section{Session 13: Lysosomes}

08:30 Michael Goligorsky

New York Medical College, USA

09:00 Jacques Mathieu

Rice University, USA

09:30 Ghezal Beliakoff

SENS Research Foundation, USA

10:00 Coffee
Cardiovascular stressors induce lysosomal dysfunction, sirtuin-1 depletion and premature senescence of vascular endothelium

Strategies for the clearance of age-related lysosomal residuals: 7-ketocholesterol and foam cell formation

Treating age-related macular degeneration through enhanced lysosomal degradation of A2E

\section{Session 14: "Aging" versus "aging-related disease"}

10:30 James Appleby

Gerontological Society of America, USA

11:00 Mike Kope

SENS Research Foundation, USA

11:30 Tanja Dominko

University of Nova Gorica, Slovenia

12:00 Lunch
The Geroscience Interest Group (GSIG): tracing pathological consequences of fundamental aging processes

Reimagine Aging

Slovenia's bid for the world stage in biogerontology

\section{Session 15: Short talks}

\section{3:15 Phil Micans}

International Antiaging

Systems, USA

13:30 Karen Kurtak

Grossman Wellness Institute, USA

13:45 Patricia d'Alessio

AISA Therapeutics/University Paris

Sud-11, France
Why orthodox medicine must change - the need for preventative/regenerative medicine

Dietary and Nutritional Manipulation of the Nuclear Transcription Factors, PPAR's and SREBP's, as a Tool for Reversing the Primary Diseases of Premature Death and Aging

Healthy aging: how stay bonny \& transcend inflammation? 
14:00 Calogero Caruso

University of Palermo, Italy

14:15 Giulia Accardi

University of Palermo, Italy

14:30 Anastasia Shindyapina

Lomonosov Moscow State University,

Russia

14:45 Josh Mitteldorf

Massachusetts Institute of Technology,

USA

15:00 Didier Coeurnelle

Heales (Healthy Life Extension Society), Belgium

15:15 Ilia Stambler

Bar Ilan University, Israel

15:30 Scott Needham

Leading Technology Group, Australia

15:45 Daria Khaltourina

Council for Public Health

and Demography, Russia

16:00 Coffee
Klotho polymorphisms and longevity: a systematic review

Insulin pathway and its correlation with ageing and agerelated diseases

Bioinformatic analysis of factors involved in age-related mineralization of soft tissues

How does the body know how old it is?

Collective consequences of a very long life. The right to life extension could / should be considered a human right.

On the history of life-extension research: Does the whole have parts?

Towards priorities for aging research

Legal problems of registering substances and therapies to cure aging

\section{Session 16: Is SENS necessary and sufficient?}

16:30 Silvia Gravina

Albert Einstein College

of Medicine, USA

17:00 Robert Shmookler-Reis

University of Arkansas, USA
Aging, single-cell methylomes

Range of variation in the duration of $\mathrm{C}$. elegans dauer and post-dauer survival: Food intake of parents and first-stage larvae affects adults recovered after 60 days of developmental arrest

17:30 Coffee

\section{Session 17: Are we rational about the quest to defeat aging?}

18:00 Alex Zhavoronkov

Biogerontology Research Foundation, UK

18:30 Mair Underwood

University of Queensland, Australia

19:00 Randall Kuhn

University of Denver, USA
New economic arguments for accelerating aging research

What reassurances do the community need regarding life extension? Evidence from studies of community attitudes and an analysis of film portrayals

International Futures: impact of decelerated or abolished aging on human demographic change 
19:30 Thomas Pyszczynski

University of Colorado, USA
Understanding the paradox of opposition to long-term extension of the human lifespan: fear of death, cultural worldviews, and the illusion of objectivity

20:00 Gala Dinner

\section{SATURDAY SEPTEMBER 7TH}

08:00

Breakfast

09:30

Punting on the River Cam

$11: 30$

Conference adjourns

Thank you for your attendance and participation! 


\section{Posters}

CeRNA bioinformatic analysis on human telomerase

Arancio W, Pizzolanti G, Giordano C

Vascular ageing: causes, mechanisms, complications and possible therapeutic strategies

Balistreri CR, Candore G, Colonna-Romano G, Forte GI, Benedetto F, Spinelli F, Ruvolo G, Caruso C, Lio D

Distinctive profile of pro-inflammatory receptors in naive and memory B cells of young, healthy elderly and Alzheimer Disease patients.

Bulati M, Buffa S, Martorana A, Gervasi F, Camarda C, Azzarello DM, Candore G, Lio D, Caruso C, Colonna-Romano G

The Human Memome Project: Text-data analytics to find socio-cultural predictors of longevity utilising the quantified self, crowd sourcing and citizen science communities

Calimport SRG, Bentley B

Identification of three particular morphological phenotypes in sporadic thoracic aortic aneurysm (S-TAA): phenotype III as S-TAA biomarker in aged individuals

Balistreri CR, Pisano C, Di Maggio FM, Scola L, Maresi E, Lio D, Caruso C, Ruvolo G, Candore G

Effect of cognitive training on the expression of brain-derived-neurotrophic-factor (BDNF) in lymphocytes of mild cognitive impairment (MCI) patients

Casoli T, Giuli C, Balietti M, Giorgetti B, Solazzi M, Fattoretti P

Longer telomeres in mitochondrial lineages doubly-selected for extreme longevity and late female reproduction

Berrett MG, Cawthon RM, Kerber RA, O'Brien E

The controversal relationship between immunosenescence and apoptosis

Ciccarelli F, De Martinis M, Ginaldi L

A distinct gene-wide pattern in LRRK2 may identify a third of Parkinson's cases Corder EH

The in vitro senescence phenotype of mesenchymal stromal cells and potential ramifications for innate immune function

Curran S, Campisi J

The Digital Ageing Atlas: Integrating the diversity of age-related changes into a unified resource Craig T, Smelick C, de Magalhaes JP

p53-dependent release of Alarmin HMGB1 is a central mediator of senescent phenotypes

Davalos AR, Kawahara M, Malhotra GK, Schaum N, Huang J, Ved U, Beausejour CM, Coppe JP, Rodier F, Campisi J

Systems Biology of Human Aging - Network Model 2013

Furber JD

Aging can be fully reversed with actual knowledge

Garcia Guerrero O

Unraveling the Gordian knot of lipofuscin

Gaspar J 
Polysaccharides from medicinal herbs as potential therapeutics for ageing and age-related neurodegeneration Li HF, Hu MH, Ma FL, Xiao LY, Zhang J, Xiang YX, Huang ZB

Protective effect of a sturgeon egg homogenate marine compound but not of EPA/DHA on arterial ultrastructure in spontaneously hypertensive rats

Bertuccelli G, Marotta F, Illuzzi N, Nagpal R, Zerbinati N, Naito Y, Mohania D, Milazzo M, Sapienza C, Italia A, Tomella C, Catanzaro $R$

Is there any beneficial effect of S. boulardi lysates on NK cell activity in otherwise healthy but immunocompromised elderly subjects?

Kantah MK, Takadanohara H, Marotta F, Illuzzi N, Naito Y, Celep G, Milazzo M, Sapienza C, Tomella C, Kobayashi R, Catanzaro $R$

Non-senescence seems feasible. But are we ready now for such possibility?

Khalyavkin AV

Whether is phenoptosis an inevitable programmed aging or it can be avoided?

Khalyavkin AV

Aging and external signaling

Khalyavkin AV, Krutko VN

Age-associated changes in walking parameters of Japanese adult women

Kikkawa K, Okada H, Oishi R

Efficiency of cellular division when creating small pockets of electric current along the walls of cells Kletetschka G, Zila V, Klimova L

Estetrol, molecular chaperones and the epigenetics of longevity and cancer resistance

Krøll J

Three-pathway model of mammalian longevity

Lehmann G, Muradian KhK, Fraifeld VE

Overview of beverages with anti-aging functions in Chinese market

Yang F, Song J, Liang M, Ma FL, Mao XL, Li HF, Huang ZB

The hidden phenomenon of oxidative stress in the treatment of subclinical hypothyroidism: a protective nutraceutical intervention

Tomella C, Catanzaro R, Illuzzi N, Cabeca A, Lorenzetti, A Celep G, Milazzo M, Sapienza C, Zerbinati N, Italia A, Bertuccelli G, Marotta F

Anti-inflammatory and anti-mutagenic effect of YHK phytocompound in hepatocytes: in view of an agemanagement liver protecting approach

Catanzaro R, Celep G, Illuzzi N, Milazzo M, Rastmanesh R, Yaduvanshi SK, He F, Solimene U, Sapienza C, Srivastava N, Marotta F

The gene expression patterns of 'normal' aging show a high similarity with age-related pathologies Mazuz K, Budovsky A, Fraifeld VE

Age dynamics of transcriptomes of long-lived Drosophila strains with GADD45 overexpression in nervous system

Moskalev AA, Plyusnina EN, Kudryavtseva AV

Soluble Tumor Necrosis Factors (sTNFR1 and sTNFR2) are increased in an age-dependent manner and in multiple organ malignancies.

Obrenovich ME, Emancipator SN, Frackelton JP, Lonsdale D 
A new telomere/stem cell theory of aging

Park E

Regeneration of neonatal lymphoid organs in the old organism Pishel IM

Intermittent hypoxia, oxidative stress and inflammation: The long and stressful road to cardiovascular disease Shannack $M$

Aged mice repeatedly injected with plasma from young mice: a survival study

Shytikov DW, Balva OV, Pishel IM, Debonneuil E

Nicolai Sietnicki - a Nicolai Fedorov follower as transhumanist precursor of a dogmatic philosophy of the extension of life concept

Sitnicki I

System modeling of genetic factors that induce pluripotency

Sukhera UA, Nawaz MS

Prediction of new lifespan regulators in C. elegans

Tacutu R, Budovsky A, de Magalhães JP, Fraifeld VE

The links between polar mRNA transport and lifespan in budding yeast Saccharomyces cerevisiae Taranukha D, Budovsky A, Gobshtis N, Braiman A, Porat Z, Aronov S, Fraifeld VE

Nutraceutical properties of extravirgin olive oil: a natural remedy for age-related disease?

Virruso C, Accardi G, Colonna-Romano G, Candore G, Vasto S, Caruso C

Diabetes type 2 and cancer: an integrative approach to prevention, treatment and rehabilitation. Voronina T, Grechko N

Web intelligence to solve aging

Wuttke D, Kulaga A

Senescent cells-bad neighbors for cancer cells?

Yanai H, Tacutu R, Budovsky A, Caliò A, Chilosi M, Fraifeld VE 


\title{
Abstracts
}

\author{
Abstracts of invited, oral and poster presentations are printed here as a single list, \\ in alphabetical order of presenting author.
}

\section{INSULIN PATHWAY AND ITS CORRELATION WITH AGEING AND AGE-RELATED DISEASES}

\author{
Accardi G, Verga S, Emanuele F, Caruso C, Virruso C, Vasto S, \\ Licastro F, Candore G \\ Laboratory of Immunopathology, Department of Pathobiology \\ and Medical and Forensic Biotechnologies, University of Palermo
}

Recent findings suggest a strong correlation between Type 2 diabetes (T2D) and Alzheimer's disease (AD). It is well known that an impairment of insulin signaling pathway can lead to insulin resistance and T2D, raising blood glucose levels. However, insulin and insulin growth factor-1 (IGF-1) contribute to neuronal survival, cognitive function and learning and memory processes as well. Indeed, many studies demonstrate that hyperinsulinemia, a pre-insulin resistance condition, is linked to higher risk of AD. Moreover, in vivo studies show an increased risk for cognitive decline in diabetic mice. The molecular explanation of this correlation is unclear but insulin resistance and impairment of insulin pathway seem to have a key role. In the brain, altered metabolism makes neurons more sensible to oxidation causing mitochondrial damages. Moreover, AD brains have a lower insulin utilization, a reduced expression of its receptors and of IGF-1, all necessary for neuronal survival and learning and memory processes. The attenuation of AKT, a kinase involved in downstream insulin cascade, causes the augmented activity of GSK-3 beta that, in mice and maybe in human, increases neuronal apoptosis and in human causes hyperphosphorilation of tau protein.

SHIP2, a 5-phosphatase, is an antagonist of PI3K and its expression, in brain of diabetic mice is increased. Moreover, its over-expression causes impairment of insulin/IGF-1 signaling, hence the decline of all related functions. This is linked to the reduction of $\mathrm{PI}(3,4,5) \mathrm{P}_{3}$ level, also in the central nervous system. Since some single nucleotide polymorphisms (SNPs) of the INPPL1, gene encoding SHIP2, in particular rs2276047, rs9886 and rs144989913, are significantly associated with T2D in European and Japanese people, we performed a case-control study with type 2 diabetic and AD Italian people. Moreover we compared these populations with younger one.

Preliminary results suggest a putative correlation between this rs144989913 and ageing rather than age-related diseases. Since this SNP is an insertion/deletion of 28 base pairs, it may cause an alteration in SHIP2 expression, confirming the evidence previous shown in old mice in which SHIP2 levels were raised.

Keywords: Alzheimer's disease, Type 2 Diabetes, Ageing, Insulin pathway, SHIP2

\section{ENGINEERING STEM CELLS FOR LIVER AND GUT REGENERATION}

Almeida-Porada G, Soland M, Boura J, Mokhtari S, Porada C Wake Forest Institute for Regenerative Medicine

Marrow stromal cells (MSC) have several unique properties, which make them well suited both for regenerative medicine and gene delivery. These include the ease of isolation and the ability to be considerably expanded in culture without losing engraftment capacity. Furthermore, MSC have been reported to reduce local inflammation, blunt immune response, and counteract the chemotactic signals released to recruit immune cells to the site of injury/inflammation.

Nevertheless, controversy still remains whether these cells, upon transplantation, would be able to be recognized in an allogeneic setting, lessening their therapeutic potential when compared with their autologous counterpart. Since MSC express negligible amounts of HLA-II, but display variable levels of HLA-I on their surface, and harbor several ligands to activating NK cell receptors, it is likely that MSC can become a target of NK and CTL. We genetically engineered MSC to express the HCMV proteins US2, US3, US6, and US11, since they were shown to reduce HLA-I surface levels on somatic cells. Moreover, this reduction in HLA-I levels prevented CTL recognition thereby preventing activation and killing of infected cells during a normal HCMV infection. We demonstrated that US6 and US11 were the most efficient in downregulating expression of HLA-I and therefore reducing MSC immunogenicity, as demonstrated by the correlation between HLA-I expression and the decrease in human PBMNC proliferation using MLR assays. We also investigated the role of the HCMV US proteins US 2, 3, 6 and 11 in protecting MSC from complement lysis, since the complement system, plays an essential role in the inflammatory process, and serves as a critical bridge between the innate and adaptive arms of the immune response. MSC express soluble factor $\mathrm{H}$, the complement regulatory proteins CD46, CD55, and CD59, that enable MSC inhibit activation of the complement system to a certain extent. Still, in the presence of inflammation and complement system activation, these innate mechanisms of protection are insufficient to prevent cellular damage and death. Expression of US2 HCMV protein on MSC had the ability to increase expression of CD46, CD55, and CD59 surface molecules, and functional assays demonstrated a significant reduction in complement lysis in MSC expressing US2 protein, when compared to the control. 
In conclusion, we are able to genetically engineer MSC to have an enhanced survival advantage in the presence of an exacerbated inflammatory microenvironment and/or in an allogeneic transplantation setting, as a result of decreased rejection by the immune system. This should extend their survival time and thereby enhance their therapeutic potential.

3

\section{IMPACT OF CR ON AGING IN RHESUS MONKEYS - A FOCUS ON METABOLISM}

Anderson RM.

Department of Medicine, SMPH, UW Madison, and GRECC VA

Hospital, Madison, WI

Caloric restriction (CR) without malnutrition delays aging and extends lifespan in diverse species; however, mechanisms have remained elusive. Furthermore, the translatability of CR to primate species, and thus the applicability of insights from CR to human health, remains an open question. Here I will discuss the longitudinal adult-onset CR study in rhesus monkeys initiated at UW Madison in the late 1980s. In this population of rhesus macaques moderate CR lowered the incidence of aging-related deaths. In addition, CR delayed the onset of age-associated pathologies. Analysis of metabolic rate and energy expenditure indicate that the CR animals differ from controls in metabolic rate and in efficiency of metabolic output. These findings are supported by serum metabonomic data that show the CR animals to be metabolically distinct from their control-fed counterparts. Subsequent studies in skeletal muscle have revealed a metabolic component to the aging process, where a shift in metabolism anticipates the onset of sarcopenia - the age related loss of muscle mass. In CR animals the onset and progression of sarcopenia is delayed and multiple indicators of cellular metabolism suggest a "younger" profile than would be expected based on chronological age. The findings from the UW-Madison study suggest that CR does slow aging in rhesus monkeys and that changes in energy metabolism are central to its mechanisms of action.

Keywords: aging, caloric restriction, rhesus monkeys, metabolism

4

\section{OUTCOMES FROM PARALLEL CR MONKEY STUDIES AT NIA AND UW MADISON}

Ingram DK, Anderson RM. Pennington Biomedical Research Center, LSU, Baton Rouge, LA, USA / Department of Medicine, SMPH, UW-Madison, and GRECC VA Hospital, Madison, WI, USA

Caloric restriction without malnutrition extends lifespan and delays the onset of age-associated disorders in diverse species, from unicellular organisms to laboratory mice and rats. Until recently, evidence of the translatability of CR's effects to human health has been a critical gap in CR research. In the late 1980s two parallel rhesus monkey caloric restriction (CR) studies were initiated to determine the effect of $\mathrm{CR}$ on resistance to illness and mortality in nonhuman primates. With more than 20 years of longitudinal data accrued, both studies have demonstrated improvements in health in CR animals compared to controls, significant in the University of Wisconsin (UW) study, and approaching significance in the National Institute on Aging
(NIA) study. The impact of CR on survival in nonhuman primates; however, is a point of departure in the two studies. In 2009 the UW-Madison-based research team reported improved survival for animals on CR. In contrast, the NIA-based research team reported in 2012 that there was no difference in survival between $\mathrm{CR}$ and Control monkeys. Here we present a comparison of key differences in study design that could explain differences in survival outcome, including the genetic origin of the study cohort, age of onset for the dietary intervention, dietary composition, feeding regimens, and protocols for animal husbandry. The relative contributions of these differences to study outcomes will be discussed, and NIA and UW perspectives on the impact of these findings on CR research and its potential to reveal insights into human health will be presented.

Keywords: calorie restriction, primate, survival, study design, genetic

5

\section{THE GEROSCIENCE INTEREST GROUP (GSIG): TRACING PATHOLOGICAL CONSEQUENCES OF FUNDAMENTAL AGING PROCESSES}

Appleby J.

Gerontological Society of America

This presentation will focus on the emerging field of geroscience, an interdisciplinary field that aims to understand the relationship between the basic biology of aging and age-related diseases. Geroscience is "coming of age." Originally coined by Gordon Lithgow, "geroscience" was just entered into Wikipedia in June of 2013. A central concept of geroscience is that multiple human diseases arise from a common cause, aging itself. Thus, slowing down the process of aging (as it has been done in animal models) represents a potentially fruitful approach to concurrently address and combat the many chronic conditions affecting the elderly.

Over the past few decades, researchers studying the biological underpinnings of the aging process have made impressive progress in understanding the genetics, biology and physiology of aging. As a result, basic research in animal models has demonstrated the plasticity of lifespan, with several genetic, behavioral and pharmacological means of extending lifespan in rodents and other model organisms. Most importantly, with some notable exceptions, extension of lifespan is accompanied by a significant delay in the appearance and progression of multiple morbidities, as well as a slowing in age-related functional decline. That is, slowing the aging processes leads to an increase in healthspan, the portion of life spent in good health.

This presentation will describe the development of the Geroscience Interest Group (GSIG), a collaborative effort across several NIH Institutes to support the development of new tools, models and paradigms that address the basic biological underpinnings of these multiple diseases. Originally conceived by Felipe Sierra, this NIH Interest Group has quickly become one of the largest such trans-NIH groups. By pooling resources and expertise, the GSIG identifies major cross-cutting areas of research and proposes coordinated approaches to identify hurdles and envision solutions to the health problems of our burgeoning elderly population. While most of the effort of the GSIG focuses on increasing awareness within and across the NIH, some activities are also open to the scientific community at large, and these will be discussed.

Keywords: geroscience, GSIG, NIH, healthspan 


\section{CERNA BIOINFORMATIC ANALYSIS ON HUMAN TELOMERASE}

Arancio W, Pizzolanti G, Giordano C.

University of Palermo

Messenger RNA (mRNA) translation efficiency is regulated by microRNAs. Each microRNA is able to regulate the translation of multiple mRNAs and each mRNA is regulated by multiple microRNAs. Thus, cellular mRNAs pool competes for microRNAs pool and viceversa. The regulatory network between mRNAs and microRNAs can be studied in the perspective of Competing Endogenous RNAs, or ceRNAs.

Here it is presented a bioinformatic study on ceRNAs for human telomerase (hTERT). Several genes potentially involved in the regulatory network of hTERT have been harvested by this study.

hTERT is essential for telomeres integrity. Telomere dysfunctions have been widely reported to be involved in Ageing, Cancer and Cellular Senescence.

Amongst the gene collected, the oncosupressor PTEN and the dynein heavy chain coding gene DNHD1 are top level interactors.

Interestingly, many genes of unknown functions result as predicted interactors, suggesting that hTERT may be involved in unexplored networks and scenarios.

Keywords: hTERT, telomerase, PTEN, dynein, CeRNA

7

\section{VASCULAR AGEING: CAUSES, MECHANISMS, COMPLICATIONS AND POSSIBLE THERAPEUTIC STRATEGIES}

Balistreri CR, Candore G, Colonna-Romano G, Forte GI, Benedetto F, Spinelli F, Ruvolo G, Caruso C, Lio D.

Immunosenescence Group, Department of Pathobiology and Medical and Forensic Biotechnologies, University of Palermo, Palermo, Italy

Ageing is increasingly considered as an independent factor for the development of cardiovascular diseases (CDs). During ageing, there are structural and functional changes in the vasculature, including dilated lumen, altered intimal-medial thickness, vascular stiffness, endothelial dysfunction, increased endothelial apoptosis, matrix metalloproteinase dysregulation, increased expression of inflammatory molecules, aggravated oxidative stress and shortened telomere length. These changes leave the body and the arteries more susceptible to hypertension, atherosclerosis, medial degeneration and the onset of a different array of artery complications (i.e. myocardial infarction, stroke, aneurysms). Metabolic syndrome, obesity and diabetes are known to accelerate ageing process and, particularly, vascular ageing. In this presentation, most of these aspects will be described in the light of recent literature data and giving particular emphasis on those, which represent object of our studies. In particular, the data discussed in this report will be based on an expert opinion derived on the findings from author studies on ageing, age-related diseases and inflammation. On the other hand, our interest will be focused in proving potential working hypotheses about possible targets for the development of strategies both for prevention and improvement of the quality of life in elderly population.

Keywords: ageing, vascular ageing, cardiovascular diseases, new therapeutic strategies

\section{ACCELERATING TRANSLATIONAL RESEARCH PROCESSES FROM BENCH TO CLINIC}

Barker RW.

Oxford-UCL Centre for the Advancement of Sustainable Medical Innovation (CASMI)

The productivity of medical innovation has been in decline, and this threatens the commitment of both public and private funders. However, there are both disruptive technologies and disruptive ideas that promise a turnaround. CASMI (www. casmi.org.uk) is exploring both, and developing testable models for change - including new open innovation-based discovery models, adaptive licensing of medicines, the use of real world data in development, and the personalisation of therapy on both genomic and behavioural grounds. With the support of SENS, CASMI is also investigating the translational issues facing cell therapy, so that the highly promising science delivers patient benefit as speedily and affordably as possible.

Keywords: CASMI, Adaptive licensing, Open innovation, Real world data, Cell therapy

9

\section{TREATING AGE-RELATED MACULAR DEGENERATION THROUGH ENHANCED LYSOSOMAL DEGRADATION OF A2E}

Beliakoff G, Yogalingam G, Goldin E. SENS Foundation Research Center

Age Related Macular Degeneration (AMD) is the leading cause of visual loss among people 65 years and older. This disease manifests into two distinct forms, wet and dry. The pathogenesis for both forms is poorly understood and numerous hypothetical models have been studied to better understand their mechanism. The dry form of AMD involves atrophy of the retinal pigment epithelium (RPE) by the accumulation of bisretinoid lipofuscin within lysosomes as the cells phagocytose the outer membranes of the photoreceptors. A major fluorescent component of RPE lipofuscin is a compound known as pyridinum bisretinoid (A2E). We have been able to degrade A2E by exogenous enzyme delivery to cultured human RPE cells and cell free systems. We have discovered an enzyme (SENS20) that, in the presence of its co-substrate, has the ability to degrade A2E in a dose-dependent manner without damaging RPE cells. This dose-dependency is evident in vitro and in cell culture assays. Our studies show evidence for SENS20's positive enzymatic activity towards synthetic A2E cultured with human RPE cells and its ability for potential treatment of dry AMD.

Keywords: age-related macular degeneration, AMD, lipofusin, A2E, retinal pigmented epithelium

10

\section{SCREENING OF MEDICINAL PLANTS IN THE JUDEA REGION (ISRAEL) FOR GEROPROTECTIVE ACTIVITIES}

Budovsky A, Shteinberg A, Maor H, Duman O, Yanai H, Wolfson M, Fraifeld VE

The Shraga Segal Department of Microbiology, Immunology and Genetics, Center for Multidisciplinary Research on Aging, BenGurion University of the Negev, Beer Sheva, Israel 
Plants growing in the Judea region are widely used in traditional medicine. This phytogeographic zone stands out in its climatic conditions and biodiversity. Consequently, both endemic and widely distributed Mediterranean plants growing in the area have unique chemotypes, characterized by accumulation of relatively high levels of phytosteroids. Our comprehensive analysis revealed that many of the plants growing in the Judea region may hold a geroprotective potential. With this in mind, we undertook a wide screen of dozens of candidate herbal extracts for their cell protective, wound healing, anti-inflammatory, and anticancer activities. The results obtained thus far have clearly shown that several of the extracts tested (i) protect normal human fibroblasts from genotoxic stress (prevent DNA double strand beaks, increase cell survival and reduce the number of cells undergoing cellular senescence); (ii) decrease secretion of pro-inflammatory cytokines; (iii) promote wound healing, and (iv) exert more pronounced cytotoxicity towards cancer cells.

This work was supported by the Israeli Ministry of Science and EC FP7 Health Research Grant number HEALTH-F4-2008202047 (RESOLVE).

Keywords: Medicinal plants, Judea region, Geroprotective activity

\section{1}

\section{DISTINCTIVE PROFILE OF PRO-INFLAMMATORY RECEPTORS IN NAIVE AND MEMORY B CELLS OF YOUNG, HEALTHY ELDERLY AND ALZHEIMER DISEASE PATIENTS}

Bulati M, Buffa S, Martorana A, Gervasi F, Camarda C, Azzarello $D M$, Candore $G$, Lio D, Caruso C, Colonna-Romano G Immunosenescence Unit, Department of Pathobiology and Medical and Forensic Biotechnologies (DIBIMEF), University of Palermo

It has been extensively demonstrated the impairment of humoral immune response in elderly humans, indeed they are characterized by a reduced ability to respond against new antigens and vaccines, due to a decrease both in percentage and absolute number of total B lymphocytes and modifications in the naïve/ memory B cell compartments. Moreover, it is also well known that elderly are characterized by an "inflamed" environment (inflamm-aging). We have previously reported the increase of a memory B cells (IgG ${ }^{+} \mathrm{IgD}^{-} \mathrm{CD}^{-} 7^{-}$, double negative, DN) population in the elderly, that are also expanded under certain pathological conditions, such as systemic lupus erythematosus (SLE), chronic HIV infection and in healthy subjects challenged with respiratory syncytial virus. In order to evaluate whether the pro-inflammatory status could influence the trafficking phenotype of naive/memory B cells, we have assessed the expression of CCR7, CCR6, CXCR3, CXCR4, CXCR5, and CD62L on naive/ memory B cell subpopulations in young, healthy elderly and in patients affected by Alzheimer Disease. Moreover, as DN B cells seem to be refractory to stimulations, we have analyzed the expression of two inhibitory receptors (CD22 and CD307d). Our data demonstrate that in the aged people, naive/memory B cell populations express a different distribution of the studied receptors that could be discussed in terms of "inflamm-ageing". Our hypothesis is that the inflammatory environment, typical of ageing, could influence the B cells trafficking rendering them more sensitive to the cytokines and chemokines produced in the elderly.

Keywords: B lymphocytes, chemokine's receptors, inflammageing, Alzheimer Disease, inhibitory receptors
12

\section{HYPOTHALAMIC CONTROL OF AGING AND LONGEVITY}

Cai D.

Albert Einstein College of Medicine

The hypothalamus is a converging point that integrates metabolic, neural, neuroendocrine, and neuroimmune signals to affect the whole body physiology. The long-standing research interest of my research is to investigate the role of neural dysregulations, in particular in terms of neural inflammation in the development of aging and aging-related diseases. Our recent observations demonstrated that the hypothalamus contains adult neural stem cells, and IKK/NF-kB activation affects the fate of these cells and cause disease consequences in relation with aging and metabolic syndrome. Taken together, we have established several conceptual models addressing the central mechanism of aging, and identified the involved mechanism that is mediated by integrated actions of neural, neuroendocrine and immune systems. We also have generated strategies for combating aging-related diseases through targeting the neural inflammatory molecular pathways. Several highlights of our recent research activities will be discussed in this symposium.

\section{THE HUMAN MEMOME PROJECT: TEXT-DATA ANALYTICS TO FIND SOCIO-CULTURAL PREDICTORS OF LONGEVITY UTILISING THE QUANTIFIED SELF, CROWD SOURCING AND CITIZEN SCIENCE COMMUNITIES}

Calimport SRG, Bentley B

Aston University

Socio-cultural markers such as attitudes, behaviours, ideas, aspirations and interests were collected and correlated to previously identified social, psychological and quantitative predictors of lifespan. Attitudes to whether a person wanted to live for as long as possible and if so in what condition were also surveyed.

The aim of the study was to identify trends, correlations and multivariate, interdisciplinary markers of health that contain word and phrase based socio-cultural markers as well as other known longevity markers. This would lead to enhanced accuracy in predictions of current health states and longevity predictions.

Text-data analysis was chosen to find novel longevity markers, as text-data is easily surveyed, available, digitized and sequence-based. As more than an analogy to The Human Genome Project, The Human Memome Project sets out to record personalised text-sequence data for individuals to be able to correlate particular text-sequences to specific health markers associated with longevity. Data was gathered from over 300 participants, 25 countries, 6 continents, in an 18-70-age range.

We have attempted to use varied statistical analysis and computational modeling techniques to build optimal sets of attitudes, behaviours and interests that correlate the most with longevity and find text-sequences that are correlated the most or least with longevity.

This project presents a proof-of-principle experiment that could be developed into advanced technology platforms, big data analysis, predictive analytics and personal health tools for 
the healthcare, preventative medicine and global, local or personal, real-time longevity risk analysis.

The Human Memome Project also presents a key engagement with the Crowd Sourcing, Citizen Science and Quantified Self communities for research into longevity.

Keywords: text, data science, markers, quantified self, citizen science

14

\section{IDENTIFICATION OF THREE PARTICULAR MORPHOLOGICAL PHENOTYPES IN SPORADIC THORACIC AORTIC ANEURYSM (S-TAA): PHENOTYPE III AS S-TAA BIOMARKER IN AGED INDIVIDUALS}

Balistreri CR, Pisano C, Di Maggio FM, Scola L, Maresi E, Lio D, Caruso C, Ruvolo G, Candore G

Immunosenescence Group, Department of Pathobiology and Medical and Forensic Biotechnologies, University of Palermo, Palermo, Italy

Ageing has a striking impact on heart and vascular system, and particularly on the large elastic arteries, i.e. aorta, determining a multitude of changes at different structural and functional levels. As result, medial degeneration (MD) occurs, which can progress in aortic dissections and rupture. A characteristic example of MD is thoracic aortic aneurysm (TAA), potentially devastating and an important source of morbidity and mortality. It happens as an isolated manifestation or defined syndromes or familial forms. Little is known about the patho-physiological mechanisms involved in sporadic TAA (S-TAA). Based on these observations, the eventual presence of typical MD morphological phenotypes were researched. Thus, histopathological and immunohistochemical analyses were assessed in aorta specimens from 100 S-TAA patients (97 men and 13 women, whose median age $62,95 \pm 11,44$ years) and 30 control individuals (20 men and 10 women, mean age: $62.9 \pm 11.57$ ) died for causes unrelated to aortic disease and no sepsis, confirmed by autopsy. Interestingly, 73 aorta tissues were with the typical MD and without atherosclerotic lesions. In these tissues, three phenotypes (I, II and III) were detected having a different quantitative relationship of cystic MD, fibrosis, apoptosis and amount of metalloproteinase- (MMP)-9. The phenotype III was mainly (63 vs. 73) observed and showed elevated cystic MD, plurifocal medial apoptosis and increased MMP-9 amount. It was significantly correlated with the severity of elastic fragmentation, hypertension, smoking, and particularly with advancing age. In contrast, no correlation was observed with aorta diameter. Thus, the phenotype III might represent the typical MD phenotype associated with major risk of aorta rupture and dissection independently on aneurysm diameter. Further studies are need to confirm these results. This might permit to assume the phenotype III with its typical histological abnormalities as optimal biomarker of rupture and/or dissection in aged individuals useful both to apply different surgical approaches or to provide appropriate surgical indications. On the other hand, an increase of S-TAA incidence in aged population has been recently observed by epidemiological studies. In addition, this might be useful, since S-TAA is clinically and predominantly a silent ailment, until rupture or dissection occurs, and insidious in its onset and progression. Furthermore, until now its diagnosis is also exclusively based on imaging technologies and no blood tests exist.

Keywords: medial degeneration, S-TAA, , histopathological and immunohistochemical analyses, phenotype III as biomarker of S-TAA in aged individuals

\section{NANOPARTICLE-BASED ARTIFICIAL RNA SILENCING MACHINERY FOR ANTIVIRAL THERAPY}

Cao YC

Department of Chemistry, University of Florida, Gainesville, FL 32611, USA

RNA interference is a fundamental gene regulatory mechanism that is mediated by the RNA-induced silencing complex (RISC). Here we report that an artificial nanoparticle complex can effectively mimic the function of the cellular RISC machinery for inducing target RNA cleavage. Our results show that a specifically designed nanozyme for the treatment of hepatitis $\mathrm{C}$ virus (HCV) can actively cleave HCV RNA in a sequence specific manner. This nanozyme is less susceptible to degradation by proteinase activity, can be effectively taken up by cultured human hepatoma cells, is nontoxic to the cultured cells and a xenotransplantation mouse model under the conditions studied, does not trigger detectable cellular interferon response, but shows potent antiviral activity against HCV in cultured cells and in the mouse model. We have observed a 99.6\% decrease in HCV RNA levels in mice treated with the nanozyme. These results show that this nanozyme approach has the potential to become a useful tool for functional genomics, as well as for combating protein expressionrelated diseases such as viral infections and cancers.

Keywords: nanozyme, hepatitis C, RNA interference, antiviral, nanoparticle

16

\section{KLOTHO POLYMORPHISMS AND LONGEVITY: A SYSTEMATIC REVIEW}

Di Bona D, Accardi G, Virruso C, Candore G, Caruso C Laboratory of Immunopathology, Department of Pathobiology and Medical and Forensic Biotechnologies, University of Palermo

Nowadays is clearly evident that genetic background constitutes integral part of successful ageing and longevity. Many studies on long lived people have been conducted emphasizing the role of certain genes in long life. Classic case-control studies, genome wide association studies and high throughput sequencing have permitted to identify a variety of genetic variants seemingly associated to longevity. Over the years, ageing research has focused on insulin/IGF-1 signaling pathway because of its evolutionary conserved correlation with life-span extension in model animals. Indeed, many single nucleotide polymorphisms (SNPs), associated with longevity were identified in genes encoding proteins that take part in this metabolic pathway. Recently we have conducted a meta-analysis that has showed the association of IGF-1R and FOXO3A polymorphisms with longevity. Closely related to this pathway is the Klotho gene. It encodes a type-I membrane protein expressed primarily in the kidney in two forms, membrane and secreted. The last form acts suppressing oxidative stress and growth factor signalling and regulating ion channels and trasporters. Its overexpression seems to be able to suppress insulin/ IGF-1 signaling extending life span as showed in transgenic mice.

Thus, our aim is to put together the results showed in literature concerning the association between SNPs of Klotho and longevity to quantify the possible effect of each single SNP and its magnitude. The results of our systematic review indicate that Klotho SNPs are associated with ageing.

Keywords: Klotho, Ageing, Systematic review, Longevity 
17

\section{EFFECT OF COGNITIVE TRAINING ON THE EXPRESSION OF BRAIN-DERIVED-NEUROTROPHIC-FACTOR (BDNF) IN LYMPHOCYTES OF MILD COGNITIVE IMPAIRMENT (MCI) PATIENTS}

\author{
Casoli T, Giuli C, Balietti M, Giorgetti B, Solazzi M, Fattoretti P \\ Neurobiology of Aging Centre Scientific Technological Area IRCCS \\ INRCA Via Birarelli 860121 Ancona Italy
}

Brain-derived neurotrophic factor (BDNF) is expressed in brain structures involved in learning and memory and is associated with neuronal plasticity by regulating growth, maintenance and survival. As BDNF plays a fundamental role in brain functioning, many studies have been performed on its possible involvement in neurodegenerative disorders such as Alzheimer's disease (AD). Indeed altered levels of BDNF have been reported in patients with $\mathrm{AD}$ and mild cognitive impairment (MCI) both in brain and blood samples. MCI is considered a transitional condition between normal aging and dementia and therapeutic treatments performed during this period have been found to be effective in delaying $\mathrm{AD}$ onset. We investigated the effect of cognitive training in improving cognitive abilities in MCI patients and healthy subjects and evaluated BDNF mRNA levels by quantitative real time reverse transcriptase polymerase chain reaction (qRT-PCR) in peripheral lymphocytes in order to identify possible associations of successful training with BDNF expression. Subjects were recruited for "My Mind Project" (code number 154/GR-2009-1584108) funded by Italian Ministry of Health and Marche Region. Each individual was randomly assigned to either a multicomponent trained exercise group (EG) or an education control group (CG) whose components received only some suggestions to improve specific outcomes with the aim to eliminate the potential bias of the intervention. After twomonth treatment EG components were evaluated and classified as "success" or "unsuccess" by improvement in scoring of some specific indicators such as objective measures of memory, attention, learning and other cognitive domains. All subjects of the four groups (EG MCI, EG healthy, CG MCI, CG healthy) were analyzed for BDNF mRNA levels at baseline and after the treatment. Preliminary results indicate that in CG there is a down-regulation of BDNF expression in MCI patients (CG MCI) and no change in healthy subjects (CG healthy) after two-month treatment. The analysis of EG shows the same trend as CG with a decrease in MCI (EG MCI) and no change in healthy subjects (EG healthy), however the decrease in BDNF mRNA levels in MCI trained subjects is more pronounced in "unsuccess" compared to "success". Our data indicate that in MCI patients a decrease of BDNF mRNA levels occurs overtime and successful cognitive training could counteract this decrement suggesting BDNF mRNA as a potential biomarker for successful cognitive training.

Keywords: BDNF, MCI, cognitive training, qRT-PCR, lymphocyte

\section{8}

\section{LONGER TELOMERES IN MITOCHONDRIAL LINEAGES DOUBLY-SELECTED FOR EXTREME LONGEVITY AND LATE FEMALE REPRODUCTION}

Berrett MG, Cawthon RM, Kerber RA, O'Brien E Dept. of Human Genetics, University of Utah, Salt Lake City, Utah 84112, USA
Successful reproduction late in life in women is associated with familial longevity. Therefore, some genetic factors may slow both overall aging and reproductive aging in females. Here we investigate mitochondrial genetic contributions to longevity in both sexes and late reproduction in women.

We identified 14,900 human mitochondrial lineages in the Utah Population Database, and independently ranked the matrilineages by longevity (the proportion of members over 65 who survived to age 90), and late fertility in women (the average age at last birth for all the mothers in each matrilineage), adjusting for longevity and late fertility observed among non-matrilineal relatives. Matrilineages in the top 5\% of the rank by longevity also appeared in the top $5 \%$ of the rank by late fertility at a rate more than 2.5 times that predicted by chance alone. This apparent lack of independence of longevity and late fertility suggests that some mitochondrial genomes may slow both whole organismal aging in both sexes and reproductive aging in women.

We collected whole blood DNA samples from 56 of the 66 matrilineages at the top of the longevity rank, and from an additional 16 matrilineal lineages that were in the top 5\% of both the longevity and late fertility ranks. Whole blood DNA samples from 48 Utah CEPH matrilineages known to be in the bottom $75 \%$ of the longevity rank served as the control group.

Measuring average telomere length in our selected matrilineages by QPCR, we found that in the mitochondrial lineages doubly-selected for longevity in both sexes and late fertility in women, the telomere length was significantly longer than in the DNA from the control mitochondrial lineages $(p=0.0000443)$. The matrilineages singly-selected for longevity also had longer, but not significantly longer, telomeres than the controls $(p=0.1175)$.

These results support the hypotheses that 1) most mitochondrial inheritance of extreme longevity in humans is not mediated by the maintenance of longer than average telomeres, and 2) some mitochondrial inheritance of longevity may involve mechanisms that robustly maintain telomere length during aging, contributing to both delayed reproductive senescence in women and slower aging generally. We are now searching for mtDNA genotypes, haplotypes, and/or other sequence features that may be enriched in these doubly-selected matrilineages, relative to the control matrilineages and the matrilineages singly-selected for longevity.

Supported by NIH/NIA grant 5R01AG038797 to Cawthon.

Keywords: telomere, mtDNA, reproduction, aging, longevity

19

\section{SIRTUIN REGULATION OF METABOLISM AND STEM CELLS}

Brown K, Qiu X, Xie S, Shin J, He M, Liu Y, Chen D University of California, Berkeley USA

The metabolic network is coordinately regulated in response to nutritional status to maintain homeostasis. Perturbed metabolic homeostasis is integral to the aging process and underlies many aging-associated diseases. Recent studies strongly suggest that metabolic enzymes are concertedly regulated via acetylation to allow coordination of the directionality and the rate of the metabolic flux upon changes in nutritional status. This mode of metabolic regulation is conserved evolutionarily and is regulated by the sirtuin family of deacetylase. SIRT3, a mammalian mitochondrial sirtuin, regulates the global acetylation landscape of mitochondrial proteins and triggers a metabolic reprogramming to reduce oxidative stress. SIRT3 regulation of oxidative stress has profound physiological relevance, such as stem cell 
maintenance and tissue homeostasis at an old age, and prevents many aging-associated diseases, including cancer, heart failure, and hearing loss. The SIRT3 regulatory program is suppressed with aging and, intriguingly, SIRT3 reactivation is an effective means of rejuvenation. SIRT7, a mammalian nuclear sirtuin, senses nutritional status and functions at the chromatin to epigenetically regulate metabolic homeostasis.

20

\section{PHAGE INTEGRASE FOR TARGETED GENE THERAPY IN MOUSE MODELS}

Qiu J, Zhang T, Zheng Q, Chen-Tsai RY

Applied StemCell, Inc. Menlo Park, CA

Phage integrase system allows integration of the transgenes into the chromosome in a site-specific manner resulting in robust, sustained transgene expression. One of the integrases, phiC31, has been used for gene therapy studies, particularly liver gene therapy in the past 10 years. In mouse models, integrases are superior to other gene integration systems including random insertion by retroviruses and transposons, or site-specific insertion systems including TALENs/ZFNs or the newest CRYSPR/ Cas9 system. Random insertion causes undesirable mutagenesis, which can activate oncogenes and initiate tumors. It can also result in silencing of transgene expression due to repressive chromosomal context or position effect. The newly developed nuclease based methods such as TALENs, ZFNs and CRYSPR/ Cas9 systems are highly effective in site-specific mutagenesis or gene knockouts; but very inefficient in gene insertion in mice. In fact, the CRYSPR/Cas9 system has yet to be tested for gene insertion in animals.

We have previously reported an improved, phiC31 integrasemediated method (TARGATT ${ }^{\mathrm{TM}}$ ) for site-specific transgenesis in mice via pronuclear microinjection, with integration efficiencies of the transgenes as high as $40 \%$. Compared to the traditional integrase methods used in liver gene therapy studies, the TARGATT $^{\mathrm{TM}}$ system achieves high gene insertion efficiency by promoting recombination between wild-type integrase recognition sites instead of pseudo sites. In addition, the TARGATT ${ }^{\mathrm{TM}}$ system confers high-level gene expression with one of the integrase recognition sites being placed in a "safe harbor" chromosome locus that is pre-characterized to be at an intergenic region, and confer high-level gene expression.

Our current study focuses on using a high-fidelity integrase, $\mathrm{Bxb1}$, to achieve gene insertion with precision. Compared to phiC31, Bxb1 only recognized its wild-type sites, but not pseudo sites, which naturally exist in mammalian genome. For example, there are at least 31 pseudo sites in the human genome and 57 pseudo sites in the mouse genome for phiC31. Using the Bxb1 integrase system, transgene insertion will only happen at the placed, wildtype site, but not at any undesirable pseudo sites in the genome. Construction of the mouse lines containing Bxb1 recognition sites in pre-selected genomic loci will be discussed. Application of the Bxb1 mouse models in gene therapy will also be discussed.

21

\section{SRF EDUCATION: TRAINING FUTURE SENS RESEARCHERS}

Chin $G$

SENS Research Foundation, USA
One of the major focuses of SRF Education is training new biomedical scientists in the SENS damage repair approach to studying the diseases and disabilities of aging. Through a collaboration with several top research institutions in the world, SRF Education has expanded its support of summer interns from seven last year to twelve this year. SRF-funded interns, both old and new, have proven to be very productive members of the SENS community. Highlights of last year's intern class include three interns being hired to continue their research at the Buck Institute for Research on Aging, one of whom published his work in the Journal of Cell Biology as part of a research group in Judith Campisi's lab.

The 2013 intern class no doubt will also make its mark on the SENS field. Several former interns are now pursuing a Ph.D. in biomedical research and those returning to undergraduate studies hope to continue SENS-related research in the future. To give a sense of the accomplishments of this year's class, one exceptional intern has been invited to give a student research talk. Additional outstanding student research projects also will be on display during the poster session.

Keywords: SENS, internships, education, aging, damage repair

22

\section{TECHNOLOGIES FOR READING, WRITING \& INTERPRETING OMES}

Church $G$

Harvard Medical School

New technologies for sequencing human diplotypes, transcriptomes, immunomes, microbiomes include Fluorescent in situ Sequencing (FISSEQ), LFR (CGI) and wearable sequencing devices (Genia). To improve interpretation of such omes from extreme youth-span individuals and to improve precision medicine requires highly integrated and comprehensive Environmental and Trait data (GET) and cohorts properly consented for global sharing (PGP). Finally, to move from correlation to causation, preventatives and therapies, we need next-generation human genome and epi-genome engineering, using CRISPR - minimizing off-target and maximizing multiplexing.

Keywords: genome, technologies

23

\section{THE CONTROVERSAL RELATIONSHIP BETWEEN IMMUNOSENESCENCE AND APOPTOSIS}

Ciccarelli F, De Martinis M, Ginaldi L

Department of life, health and environmental sciences, University of L'Aquila, Italy

Programmed cell death or apoptosis is a complex process that allows cells to die in a controlled fashion, On the basis of the nature of the apoptosis-inducing stimuli, an activation-induced apoptosis and a damage-induced apoptosis can be identified. The balance between removal of damaged cells via apoptosis and proliferation of needed cells is a central process in body homeostasis and its derangement is involved in many physiopathological conditions, including ageing and immunosenescence. Programmed cell death is a key regulator of clonotypic diversity generation during lymphocyte ontogenesis and is essential for the proper function of the immune system, 
controlling lymphocyte activation and clonal expansion following antigenic stimulation. Moreover, apoptosis is markedly involved in many changes characteristic of immunosenescence, such as thymic involution, alteration of T-cell repertoire, accumulation of memory/effector cells, osteoporosis, autoimmunity and cancer. Despite the increasing data on age-related changes in cell-death phenomena, the impact of apoptosis remodelling in the ageing process and its exact role in the pathogenesis of agerelated diseases are still controversal. The remodulation of apoptotic processes observed in the elderly could exert variable impacts on ageing and human health. Immunosenescence, i.e. the ageing of the immune system observed in the elderly, is characterized by a peculiar remodelling of programmed cell death phenomena. On the other hand apoptosis plays a central role in driving the ageing process. Both recurrent antigenic stimulations and oxidative metabolism byproducts, impinging upon the immune system, modify the apoptotic capability of lymphocytes, driving immunosenescence. In addition to the upregulation of anti-stress responses and inflammatory cytokines (inflammageing), the subtle remodelling apoptotic pathways in the elderly contributes to the phenotypic and functional characteristics of the aged immune system, representing one of the major determinants of ageing rate and longevity, as well as of the most common age-related diseases. Understanding of apoptosis signalling mechanisms in more details is therefore essential for the identification of multiple potential targets to inhibit or enhance apoptosis in different cell subsets. A correct modulation of apoptosis in specific lymphocyte subsets could preserve immune function in the elderly and may be useful for prolonging the lifespan and reducing age-related degenerative, inflammatory and neoplastic diseases, contributing to successful ageing.

Keywords: Apoptosis, Activation-induced cell ceath, Damage-induced cell death, Immunosenescence, Ageing

COLLECTIVE CONSEQUENCES OF A VERY LONG LIFE. THE RIGHT TO LIFE EXTENSION COULD / SHOULD BE CONSIDERED A HUMAN RIGHT

Coeurnelle D

Heales (Healthy Life Extension Society)

A longer and healthier life is enjoyed by the citizens who can benefit from it. This evolution is also positive for the whole society. It is better for the economy, for a sustainable environment, for a peaceful planet, for the level of well-being in the society. This speech gives a description of positive political, economic and sociological aspects of a world with a largely delayed senescence. The question of the moral necessity of health research funding will be discussed. Should the State subsidize life extension? Taking in consideration, medical progresses and the possibilities to accelerate them, we can consider the right to health as defined in national laws, national constitutions and international treaties under a new light.

Keywords: Public funding of life extension, Collective advantages of life extension, Human rights

LOW RISK FOR CORONARY ARTERY DISEASE AND FINDINGS IN THE TERC REGION ON 3Q26

Corder $E H$

Matrix Genomics, Inc.
The TERC region on chromosome $3 q 26$ was investigated for British coronary artery disease patients $(n=1487)$ and for UK blood service donors $(n=1430)$. The aim was to identify regionwide patterns associated with telomere length and their prevalence in the two subject groups. The 131 SNPs distributed over 200,000 bp were represented by 41 variables, either SNP genotype or multilocus genotype for highly correlated SNPs; quantile of leucocyte telomere length (T/S ratio adjusted for age and sex) was used in model estimation. Maximum likelihood was employed to identify region-wide patterns associated with telomere length (fuzzy latent classification; GoM). Three essentially identical region-wide patterns labeled as I, II and III were identified in both samples. Mean adjusted telomere length was $1.84(n=398), 2.03(n=148)$, and $1.85(n=325)$ for blood donors who genetically matched the respective patterns, $1.59(n=369)$, $1.67(n=46)$ and $1.60(n=321)$ for the heart patients. Few heart patients matched favorable pattern II ( $3 \%$ vs $10 \%, p<0.001)$, implying protection for coronary heart disease. However, these patients had only marginally longer telomeres as compared to other patients, implying that telomere attrition itself is relevant to coronary artery disease. Considering raw unadjusted T/S found for blood donors who matched one of the patterns, the rate of age-related attrition was small $\sim 20 \mathrm{bp}$ annually for I, II and III. Instead, extrapolating back to birth in linear models, there were large differences in telomere set point: 1.836 ( $\sim 7840 \mathrm{bp}$ ) for pattern I, 2.089 ( $\sim 8920 \mathrm{bp})$ for pattern II, and, $1.802(\sim 7695 \mathrm{bp})$ for pattern III. We conclude that a specific genetic pattern in the TERC region found for $\sim 10 \%$ of the British population predisposes to good health and longevity. The alterations in LRRIQ4 and LRRC31specific to pattern II are of great interest.

Keywords: grade-of-membership analysis, coronary artery disease, TERC gene, telomere length, telomere set point

26

\section{A DISTINCT GENE-WIDE PATTERN IN LRRK2 MAY IDENTIFY A THIRD OF PARKINSON'S CASES}

Corder $E H$

Matrix Genomics, Inc.

Parkinson's disease is becoming tractable. Risk is robustly associated with a short list of genes: BST1, CCDC62/HIP1R, DGKQ/GAK, GBA, LRRK2, MAPT, MCCC1/LAMP3, PARK16, SNCA, STK39, SYT11/RAB24, and ITGA8 (Lill et al, 2012). Allelic frequencies for multiple SNPs located in these genes differ among PD cases as compared to control groups derived from the general population. However, association is a long way from an adequate description of risk and causation. We investigated detailed information on the LRRK2 gene obtained from members of the NINDS cohort (http://ccr.coriell.org/ Sections/Collections/ NINDS/) in order to identify gene-wide patterns associated with PD: 275 cases presenting at age 60 or older and an equal number of neurologically healthy control subjects having a similar age and sex composition. All 51 exons were sequenced as well as at least $50 \mathrm{bp}$ of flanking intronic sequence. A total of 84 SNPs had a minor allele count of GE 5. These identified 49 variables represented by genotype or multilocus genotype (for highly correlated SNPs). Maximum likelihood was employed to identify gene-wide patterns (fuzzy latent classification; GoM). Three patterns were identified labeled as I, II and III. I (high risk) was distinct and represented by specific heterozygous or novel genotypes located throughout the gene in multiple functional domains. II (low risk) and III (high risk) lacked these genotypes. 
These patterns were alike except that III had some probability of coding mutations and minor alleles at rs33958906. Many subjects matched I, II, \& III: cases: 46, 4, 137; controls: 0, 178, 0. Most subjects could be assigned to one pattern based on GE $50 \%$ similarity: cases: 91, 4, 178; controls: 27, 250, 0. We conclude that a third of PD cases have a specific gene-wide pattern of alterations in the LRRK2 that may be a sufficient basis for PD. This work needs to be replicated and integrated into a unified risk model involving all the listed genes.

Keywords: grade-of-membership analysis, LRRK2, genetic epidemiology, Parkinson's disease

27

\section{HEALTHY AGING: HOW STAY BONNY \& TRANSCEND INFLAMMATION?}

d'Alessio P

AISA Therapeutics and University Paris Sud-11

Introduction: People are driven by their obsession to stay young, and most importantly, in a visible way. Promising healthy aging, the market of dietary supplements is growing. Inflammation is a strategic key element in major degenerative diseases and has stimulated research on "inflammaging". It is also recognized that inflammation contributes to depression. How can inflammation be managed without treating it with anti-inflammatory drugs? Pro- or prebiotics have been shown to directly influence the microbioma, while natural compounds may reduce the symptoms of gut inflammation. To avoid gut colitis, various herbal remedies have been reviewed by S. Danese (Gut, 2011). Also, the buccal area and its specific microbiota considerably influence the global status of the digestive system and thus of the whole organism (N. Illuzzi). Moreover, on the base of stool analyses available now (www.labo.lu) pharmacogenetics is increasingly entering the field of nutrition, aiming at the prediction and optimization of individual profiles of gut microbiome and immune-inflammatory-clinical responses to therapy. $\mathrm{Nu}$ trigenetics may have even greater potential. In many cases, modulating cytokine responses and changing the gut microbiota can be obtained by an elemental diet

Material and methods: Analyses correlating dietary intake with systemic markers of inflammation as high-sensitivity Creactive protein (HS-CRP), Interleukin-6 (IL-6), and Tumor Necrosis Factor alpha (TNF- $\alpha$ ) have been performed by us in the Ristomed study (www.ristomed.com). The multi-centric Ristomed study (PRIME and www.ristomed.eu) concentrated on healthy subjects in the range of 65-85 years. The tri-terpene tripterine, a chemical compound of Tripterygium woolfordii hoek a Chinese plant eaten in the soup, displays anti-inflammatory properties and prevents arthritis.

Results: Monoterpens of herbal origin (as identified by AISA Therapeutics) turned out to modulate the expression of Il- 6 and improve mood. Results on hand grip improvement were correlated with anti-depressive effects of AISA 5203-L (Prime).

Conclusions: It seems that inflammation so heavily involved in aging progress and mood change can be best managed acting on diet because of the consequences of the microbiome stabilization. Supplementation by specific herbal compounds characterized for focused action on inflammatory markers and / or signaling, may enhance this anti-inflammatory properties of a diet.

References:

d'Alessio P. et al. Tripterine inhibits the expression of adhesion molecules in activated endothelial cells. Journal Leuko Biol, vol 80, p. 309-319, 2006.
Danese S. New therapies for inflammatory bowel disease: from the bench to the bedside. Gut. 2012 Jun;61(6):918-32. doi: 10.1136/gutjnl-2011-300904. Epub 2011 Nov 23. Review.

d'Alessio P. et al. Gender differences in response to dietary supplementation by Orange Peel Extract in elderly people in the Ristomed study: impact on Quality of Life and inflammation. Prime 2012; 2:30-7.

d'Alessio P. et al. Oral administration of $d$-Limonene controls inflammation in rat colitis and displays anti-inflammatory properties as diet supplementation in humans. Life Sciences, Vol. 92, 24-26, 10 July 2013, 1151-1156.

Keywords: inflammation, supplementation with anti-inflammatory molecule, low grade inflammation in the elderly, ristomed EU study, anxiety and depression

\section{8}

\section{SEQUENCING THE GENOME OF THE LONGEST-LIVED MAMMAL TO IDENTIFY LONGEVITY ASSURANCE MECHANISMS}

\section{de Magalhaes JP \\ University of Liverpool}

The bowhead whale (Balaena mysticetus) has not only been estimated to live over 200 years, making it the longest-lived mammal, but these animals remain disease-free until much more advanced ages than humans can. The mechanisms for the longevity and resistance to aging-related diseases of bowhead whales are unknown, but it is clear they must possess aging prevention mechanisms. In particular in the context of cancer, bowhead whales must have anti-tumour mechanisms, because given their large size and longevity their cells must have a massively lower chance of developing into cancer when compared to human cells. In this project, we are sequencing and analyzing the genome of the bowhead whale to identify longevity assurance mechanisms. We are also performing analyses to identify promising candidate genes for further study and identify possible mechanisms that may explain the long lifespan and resistance to age-related diseases of bowhead whales. Overall, this project will provide a key resource for studying the bowhead whale's exceptional longevity and resistance to diseases. Studying a species so long-lived and with such an extraordinary resistance to age-related diseases will help elucidate mechanisms and genes conferring longevity and disease resistance in mammals that in the future may be applied to improve human health.

29

\section{THE DIGITAL AGEING ATLAS: INTEGRATING THE DIVERSITY OF AGE-RELATED CHANGES INTO A UNIFIED RESOURCE}

Craig T, Smelick C, de Magalhaes JP

Integrative Genomics of Ageing Group, University of Liverpool

Human ageing is characterised by multiple changes at different levels of biological organisation. It is still not clear which (if any) molecular, cellular or physiological changes are more important drivers of the process of ageing or how they influence each other. One difficulty in understanding how different processes at different scales relate to ageing as a whole is the lack of integrative, holistic views of ageing. Even though studies characterising the ageing phenotype have been conducted for decades, there is no centralised resource in which data on multiple 
age-related changes are collated. To address this and facilitate integrative, system-level studies of ageing we developed the Digital Ageing Atlas (DAA). The DAA is a one-stop collection of human age-related data covering different biological levels (molecular, cellular, physiological, psychological and pathological) that is freely available online (http://ageing-map.org/). Besides, not only does the DAA merge different types of data into a single repository, but we developed an intuitive and userfriendly web resource that allows accessing, searching, browsing and retrieving the datasets in an integrated and interactive fashion. Overall, the DAA offers a new approach to systemising ageing resources, providing a manually-curated and readily accessible source of age-related changes.

\section{0}

\section{SLOVENIA'S BID FOR THE WORLD STAGE IN BIOGERONTOLOGY}

\section{Dominko $T$}

University of Nova Gorica

Managing the process and changes that occur with aging is a complex challenge that will require much more than cutting edge research in biomedical sciences. It is only with an approach that marries different fields of science with environment and nutrition, that we will truly be able to alleviate the effects of aging. It becomes important to integrate various disciplines that traditionally do not align themselves along common goals: including molecular biology, genetics, physiology, nutrition, biochemistry, endocrinology, psycho-physiology and movement science in order to prevent the development of disease and slow the aging process. This form of medicine focuses not just on diseases and symptoms but rather on a combination of physiological, biochemical, genetic and environmental factors underlying degenerative process.

Within century-old traditions of vineyards, exploring the effects of wine and food on healthy aging together with cutting edge science in Slovenian Vipava valley lays an opportunity to capitalize on this new and unique concept. We stand on the verge of a revolution in medicine: understanding, treating, and ultimately preventing the causes of degenerative aging. ICAR will become the hub for generation and sharing of research findings, it will provide new graduate training opportunities, and will become a prime European site for extensive training across a broad spectrum of life science areas via summer courses and internships, residential visiting scholar opportunities, scientific meetings and conferences. Located within the renovated historic $17^{\text {th }}$ century palace Lanthieri complex, the Center will unify science, education, corporate engagement and public outreach to achieve this common goal. Where sun, soil and tradition join science - for active and healthy aging.

\section{1}

\section{WILL CALORIE RESTRICTION WORK IN HUMANS?}

\section{Fontana $L$}

Washington University in St. Louis, USA \& University of Salerno, Italy

Calorie Restriction (CR) without malnutrition slows aging and increases lifespan in simple model organisms and rodents. In Rhesus monkeys long-term CR reduces the incidence of type 2 diabetes, cardiovascular disease and cancer, and protects against age-associated sarcopenia and neurodegeneration. However, only in the Wisconsin, but not in the NIA monkey study, CR significantly increased average lifespan so far. Major differences in diet composition and study design between the 2 on-going trials may explain the discrepancies in survival and disease incidence between the Wisconsin and NIA CR monkey studies. However, many of the metabolic and hormonal adaptations that are typical of the long-lived CR mice and rats did not occur in either the NIA or WNPRC CR monkeys. Whether or not CR will extend lifespan in humans is not known yet, but accumulating data indicate that moderate $\mathrm{CR}$ with adequate nutrition has a powerful protective effect against obesity, type 2 diabetes, inflammation, hypertension and cardiovascular disease, and reduces metabolic risk factors associated with cancer. Moreover, $\mathrm{CR}$ in human beings improves left ventricular diastolic function and heart rate variability, two markers of cardiovascular aging, and induces dramatic modifications of the skeletal muscle transcriptional profile that resemble those of younger individuals.

Keywords: calorie restriction, human, lifespan

\section{2}

\section{MYELIN REGENERATION AND AGEING}

Franklin RJM

Wellcome Trust-MRC Cambridge Stem Cell Institute, University of Cambridge

Remyelination, the process by which new myelin sheaths are restored to demyelinated axons, represents one of the most compelling examples of adult multipotent stem cells contributing to regeneration of the injured CNS. This process can occur with remarkable efficiency in multiple sclerosis (MS), and in experimental models, revealing an impressive ability of the adult CNS to repair itself. However, the inconsistency of remyelination in MS, and the loss of axonal integrity that results from its failure, makes enhancement of remyelination an important therapeutic objective. There is now compelling evidence that ageing is the major contributor to the declining efficiency of remyelination and that this is largely due to a failure of stem cell differentiation. This talk will review recent studies we have undertaken aimed at obtaining a detailed understanding of the mechanisms of regulating differentiation during remyelination and hence identifying novel therapeutic targets.

Keywords: myelin, demyelination, remyelination, stem cells, aging

33

\section{INDUCING EXOCYTOSIS TO REMOVE LIPOFUSCIN}

Furber JD

Legendary Pharmaceuticals (www.LegendaryPharma.com)

In old age, redox-toxic lipofuscin clogs lysosomes in critical nondividing cells. This blocks autophagy and cell maintenance, impairs cell functions, and can kill essential cells. Inducing exocytosis of lysosomes could clear toxic lipofuscin out of the cell, allowing autophagy and cell repair to restore the cell to a more youthful, healthy state. Induction of lysosomal exocytosis has been demonstrated in human cells. The signaling pathways are being mapped. We are testing methods to safely induce exocytosis of lipofuscin-loaded lysosomes in old cells.

Keywords: lipofuscin, lysosome, exocytosis, autophagy, rejuvenation 
34

\section{SYSTEMS BIOLOGY OF HUMAN AGING - NETWORK MODEL 2013}

Furber JD

Legendary Pharmaceuticals www.LegendaryPharma.com

This network diagram is presented to aid in conceptualizing the many processes of aging, the causal chains of events, and the interactions among them. Contemplation of this network suggests promising intervention points for therapy development. This diagram is maintained on the Web as a reference for researchers and students. Content is updated as new information comes to light. www.LegendaryPharma.com/chartbg.html

At first glance the network looks like a complicated web. However, as a conceptual summary, in one view, we can see how most biogerontological processes relate to each other. Importantly, examination of these relationships allows us to pick out reasonably plausible causal chains of events. Within these chains, we can see age-related changes or accumulations that appear to be promising targets for future therapy development.

The many observable signs of human senescence have been hypothesized by various researchers to result from several primary causes. Inspection of the biochemical and physiological pathways associated with age-related changes and with the hypothesized causes reveals several parallel cascades of events that involve several important interactions and feedback loops. This network model includes both intracellular and extracellular processes. It ranges in scale from the molecular to the whole-body level. Effects due to externalities, lifestyle, environment, and proposed interventions are highlighted around the margins of the network.

Several researchers have proposed to adapt the network model's contents into an interactive website with hyperlinks to references and background materials. A symposium to explore this development was held at Arizona State University, December 2008; abstracts are at

http://legendarypharma.com/meetings/2008ASU_SysBioAging/aging.html

Keywords: causes of aging, systems biology of aging, targets for drug development, flow chart of aging

\section{AGING CAN BE FULLY REVERSED WITH ACTUAL KNOWLEDGE}

Garcia Guerrero O

Hiperproteina S.L

Aging is just a matter of resouces. If more resources go into a system than the leave the system rejuvenates, if the enter is equal to exit the system stay the same, if exit is bigger than the enter the human gets old. From resources we can undestand micronutrients, lack of demanding condition.

The human body has all the technology to deal with all kind of damage incluiding mitochondrial dna repair and tranfer of mitochondria and lysosomes throught tunneling nanotubes from stem cells.

Human extract the best from them in order to make sperm or breast milk.

Breast milk is the most charged meal for humans, incluiding Ribonucleotides, Deoxyribonucleotides, Creatine, Spermidine, Spermine, Choline, MyoInositol

Tetrahidrobiopterin, Sialic Acid, Fucose.....
For rejuvenation a men is a simple as stop as possible to ejaculate (retain resources) and drink a product with similar ingredients as breast milk.

There are hundred or more people doing research about aging we must put in doubt that the really want to repair aging, as I think thay the are afraid of losing their job.

36

\section{UNRAVELING THE GORDIAN KNOT OF LIPOFUSCIN}

\section{Gaspar J}

Rice University, 6100 Main St., Houston, TX 77005

Lipofuscin accumulates within the lysosomes of many healthy human cell types as one ages. The mitochondrial axis theory of aging suggests lipofuscin originates from incomplete mitophagy. As mitochondria die, they must be recycled via the lysosome. Lipofuscin contains roughly $25 \%$ mitochondrial proteins, and it is believed lipofuscin is simply the highly oxidized remnants of incompletely turned over mitochondria. The lipofuscin becomes trapped within the lysosome, rendering it nonfunctional. As more lipofuscin accumulates within the lysosomes of a healthy cell, the cell's endosomal/lysosomal pathway starts to become less efficient at removing toxins from the cell and overall cell health declines. Analogous to a car accident on a freeway that leads to hours of commuter backup, so too, as lipofuscin builds up within the lysosomes does it create a backlog of other waste products that need degraded within the cell. Many major health-related illnesses are related to aggregate protein accumulation including Alzheimer's disease, cardiovascular disease and age-related macular degeneration. Here we document the efficacy of a small molecule activator at removing lipofuscin generated by chemical means in human fibroblasts and explore how lipofuscin removal affects mitophagy. In addition, we explore the homology between lipofuscin generated by different physiological stressors.

\section{7}

\section{EFFECT OF SENESCENCE ON BONE REMODELLING: THE ROLE OF INFLAMMAGEING}

Ginaldi L, Ciccarelli F, De Martinis M

Department of life, health and environmental sciences, University

of L'Aquila, Italy

Osteoporosis is a major cause of morbidity and mortality in older people. Osteoporosis is characterized by low bone mass and microarchitectural deterioration of bone tissue with a subsequent increase in bone fragility and susceptibility to fractures. There are a large number of risk factors for the development of senile osteoporosis. However, recent discoveries suggest that inflammageing, i.e. the immunological inflammatory profile characterizing immune senescence, plays a major role in bone remodelling through pro-inflammatory cytokines, which are able to influence osteoclasts and osteoblasts. Therefore ageing induces osteoporosis through immunologically mediated modulation of bone remodelling. It is the activated immune profile which, through inflammation and inflammatory cytokine production, modulates osteoblast and osteoclast activity leading to osteoporosis. Senile osteoporosis is an example of the central role of immune-mediated inflammation in determining bone resorption. Here we discuss the pathogenesis of osteoporosis in the context of immunosenescence and focus on therapeutic strategies targeting osteoimmunology pathways. Osteoimmunology is an emerging research area that 
deals with the mutual interactions between bone and the immune system. Osteoclast, the sole resorbing bone cell, is the center of attention in osteoimmunological research, due to its haematopoietic origin and strong activation through cytokines. However, novel functions of osteoblasts, specialized in bone formation, and osteocytes, the structural bone cells, have also been recently unraveled. Critical proteins with osteoimmune functions, including the pro-osteoclastic cytokine receptor activator of NF- $\kappa \mathrm{B}$ ligand and inhibitors of the pro-osteoblastic Wnt signaling pathway, recently showed roles of paramount importance in both inflammageing and osteoporosis. The combined effects of estrogen deprivation and raising of FSH production occurring in menopause cause a marked stimulation of bone resorption and a rapid bone loss which is central for the onset of postmenopausal osteoporosis and chronic inflammatory diseases. These discoveries have also led to the development of targeted therapies to counteract not only inflammation-induced bone loss but also postmenopausal and senile osteoporosis. Ageing also affects bone through the production of myeloid cells in bone marrow, a condition associated with increased chronic inflammation by macrophages and decreased bone mass due to excess bone resorption by osteoclasts. The ageing of both haematopoietic stem cells and bone marrow microenvironment, emerging from increased oxidative stress, peroxisome proliferator-activated receptor $\gamma$ activity and adipogenesis in the ageing bone marrow, contribute to impaired osteoblastogenesis and niche cell function. Anti-ageing interventions targeting mesenchymal stem cell differentiation in the bone marrow could enhance osteoblastic bone formation and counteract inflammation and osteoclastic bone loss. The inducer cells in senile osteoporosis are immune cells, such as activated macrophages and lymphocytes, which produce cytokines and soluble mediators able to stimulate osteoclast differentiation and activation. Molecules that regulate osteoclastogenesis are key factors in many immunological functions. Osteoporosis and inflammageing share the same immunological profile. Osteoclastogenesis and inflammation are directly proportional to osteoclast precursor levels in peripheral blood. Characteristic of an agedi mmune profile is the accumulation of activated memory cells expressing RANKL, preferentially resident in the bone and secreting osteoclastogenic proinflammatory cytokines. The immunophenotypical analysis of peripheral blood lymphocyte subsets confirms the deep involvement of the immune system in bone remodelling. CD3 + T lymphocytes are increased in osteoporotic patients, as well as their CD4 + /CD8 + ratio, whereas CD20 + B lymphocytes are significantly decreased. Moreover, the CD8+CD56+ lymphoid subset including killer/effector lymphocytes producing large amounts of the inflammatory cytokine TNF- $\alpha$, is also expanded. In osteoporotic patients, as well as in elderly people, there is an increase in $\mathrm{CD} 45 \mathrm{RO}+$ memory lymphocytes, whereas the CD45RA + naive subset is markedly decreased. Another important mechanism which could link inflammageing and osteoporosis is the dysregulation of T-regulatory cells producing soluble factors IL-10 and TGF- $\beta$, both able to antagonize immune media tedbone resorption.

Keywords: Bone remodelling, Osteoporosis, Inflammageing, Immunosenescence, Ageing

\section{8}

\section{CARDIOVASCULAR STRESSORS INDUCE LYSOSOMAL DYSFUNCTION, SIRTUIN-1 DEPLETION AND PREMATURE SENESCENCE OF VASCULAR ENDOTHELIUM}

Goligorsky MS

New York Medical College Valhalla, NY, USA
Chronic cardiovascular and kidney diseases are associated with premature vascular senescence, which contributes to vasculopathy and accelerated progression of original malady. At least three independent pathways participate in this predilection to premature vascular/endothelial senescence, as will be discussed in the presentation.

1. Frustrated autophagy pathway. This pathway can be initiated by each of the following non-traditional cardiovascular risk factors: asymmetric dimethylarginine (ADMA), advanced glycated end-products (AGEs), or a mimic of oxidative stress, hydrogen peroxide. Each leads to a rapid loss of integrity of the lysosomal membrane and collapse of lysosomal $\mathrm{pH}$ gradient. The latter results in impaired digestion of phagocytosed organelles or proteins within phagolysosomes, their engorgement, and accumulation of non-degraded materials destined for degradation.

2. Sirtuin-1 depletion pathway. The same cardiovascular risk factors lead to depletion of endothelial sirtuin- 1 and premature senescence of endothelial and endothelial progenitor cells. The mechanism of this depletion involves leakage of cathepsins, which directly degrade sirtuin-1.

3. Putative amino acid-mediated mTOR pathway. Endothelial cell dysfunction induced by the above cardiovascular risk factors is associated with profound perturbations in cell metabolism. Proteomic analysis of dysfunctional endothelial cells reveales depletion of 2 mitochondrial enzymes of the Krebs cycle, namely, enoyl-CoA-hydratase and aconitase-2, consequently leading to a switch to the Warburgtype metabolism, normoxic glycolysis. Metabolomic profiling of microvasculature obtained from mice with chronic endothelial dysfunction reveales depletion of glutamine. It is possible that glutamine deficiency is responsible in part for the activation of mTOR, resulting in turn in further inhibition of autophagy and premature endothelial senescence.

Elucidation of these pathways of premature vascular senescence helped to design rational therapeutic strategies: 1) these pathways can be disrupted by antioxidants/peroxinitrite scavengers; 2) cathepsin inhibitors; and 3) supplementation with glutamine, resulting in the restoration of endothelial cell functions.

Keywords: lysosomal membrane permeabilization, autophagy, metabolome, endothelial dysfunction, sirtuin-1than

\section{9}

\section{AGING, SINGLE-CELL METHYLOMES}

Gravina S, Ganapathi S, Akman K, Tresch A, Vijg J Department of Genetics Albert Einstein College of Medicine, 1301 Morris Park Ave Bronx, New York 10461

Recent data suggest that the epigenome is highly dynamic and serves as an interface between the environment and the inherited static genome. The large volume of epigenomic events and its continuous need of maintenance, i.e., after DNA repair or replication, suggest a high chance of errors. The question we wish to address is how unstable the epigenome really is. Do epimutations accumulate with age and do they occur in a random fashion, i.e., as 'epigenomic drift'? Do they ever reach levels that are high enough to have functional consequences?

To experimentally determine epigenetic drift we focused on DNA methylation, a major layer of epigenomic control. To study intra-organ variation in DNA methylation during aging, it is necessary to have access to procedures that allow assessing DNA methylation patterns at the level of single-cells or 
single-molecules. Such methodology is currently entirely lacking: to fill this void we have optimized bisulfite sequencing for single cell analysis.

The procedure developed allows us to analyze DNA methylation patterns in single cells, within promoter regions of genes or genome-wide. Data on mouse fibroblasts, neuronal nuclei and hepatocytes will be presented and discussed. We are currently applying the method to test the hypothesis that random DNA methylation changes accumulate in the mouse liver during aging, contributing to functional decline of somatic cells that gives rise to chronic pathology and aging.

40

\section{WHAT GOOD IS AN OLD BRAIN IN A YOUNG BODY - A STRATEGY FOR REGENERATING THE NEOCORTEX}

McKeehan N, Diaz F, Kang W, Hébert JM

Albert Einstein College of Medicine, Bronx, New York, United States of America

The neocortex is the seat of our highest cognitive functions. Neocortical projection neurons, the principle neurons of the neocortex, become dysfunctional with age and can be lost due to neurodegeneration or insults such as stroke or trauma. The highly plastic nature of neocortical neuronal networks suggests that they could in theory withstand a slow turnover of neurons over time without significantly compromising function or memory. Therefore cell replacement may be a viable approach to rejuvenating the neocortex. We have recently shown that endogenous stem or precursor cells have little or no ability to replace projection neurons when they are lost in the adult neocortex (Diaz et al., April 2013, J. Neuroscience). Moreover, previous attempts at replacing these projection neurons using several types of transplanted neural stem or precursor cells have not provided viable strategies. One limitation of previous attempts is that the transplanted cells remained primarily in the transplant site or only migrated a short distance away from it. Therefore, to achieve the goal of functionally integrating new projection neurons throughout broad areas of the neocortex and to minimize the number of injection sites, a novel strategy is required that takes into account cell dispersion. Our goal is to develop an approach for introducing new, widely dispersed, projection neurons in the adult neocortex, providing a paradigm for testing whether they can functionally integrate. One way in which we are attempting to accomplish this is by using embryonic cells that are inherently migratory when transplanted into the adult mouse neocortex. However, because these particular migratory precursor cells generate interneurons rather than projection neurons, we engineer them with lentiviruses with which we can induce expression of transcription factors that will reprogram them to the desired fate once they have dispersed.

Keywords: neocortex, cell replacement, neurons

41

\section{MOLECULAR MECHANISMS OF TELOMERASE REGULATION IN GENETICALLY DEFINED HUMAN STEM CELL MODELS}

Hockemeyer D

Department of Molecular \& Cellular Biology, University of California, Berkeley

The essential role of telomerase in stem cell maintenance has long been recognized. Nevertheless, we still have little under- standing of the molecular mechanisms by which human cells regulate telomerase activity to ensure tissue homeostasis and how its dysfunction can lead to aging and tumorigenesis. The natural regulation of telomerase activity in human tissue and the impact of telomere shortening on untransformed human cells can only be studied in a primary human stem cell system. However, until recently, technical limitations, including the inefficiency of genetic manipulation, have impeded the use of human stem cells as research tools. We have overcome this by establishing the use of site-specific nucleases to genetically engineer human pluripotent stem cells (hPSCs). This technology allows us, for the first time, to investigate the two key regulatory events of human telomerase function in a genetically defined human stem cell system: its transcriptional regulation and the recruitment of the telomerase holoenzyme to telomeres.

Keywords: ZFN, TALEN, telomerase, stem cells, site-specific nuclease

\section{2}

\section{POLYSACCHARIDES FROM MEDICINAL HERBS AS POTENTIAL THERAPEUTICS FOR AGEING AND AGE-RELATED NEURODEGENERATION}

\author{
Li HF, Hu MH, Ma FL, Xiao LY, Zhang J, Xiang YX, Huang ZB \\ School of Biosciences and Biopharmaceutics, Guangdong \\ Pharmaceutical University
}

Ageing and age-related disorders have increasingly become a global issue in social and medical sciences. Although none of the existing ageing theories can give full explanation by itself to the ageing process, a number of studies have uncovered important ageing/anti-ageing clues, such as free radicals, inflammation, telomere and lifespan-regulating pathways. Recent studies have provided advances in age-related disorders, e.g. involvement of protein misfolding and abnormal aggregation in age-related neurodegeneration. Accordingly, strategies to slow ageing process may provide efficient means to delay and/or prevent age-related disorders. In fact, anti-ageing Chinese herbs have been widely used to treat age-related diseases. For instance, many polysaccharides from medicinal herbs exhibit considerable antioxidant and anti-inflammation capabilities in cellular and animal models and play a beneficial role in ageing and associated diseases. Some polysaccharides can reduce cellular senescence and modulate lifespan of animal models via such mechanisms as telomere length and insulin signaling pathway. A number of polysaccharides have recently been shown to have inhibitory effect against protein aggregation and aggregation-associated neurotoxicity. Nevertheless, the anti-ageing and neuroprotective functions of polysaccharides are much less investigated and poorly understood as compared to the extensive studies of their antitumor and immunomodulatory effects. Here we attempt to present the current status of polysaccharides in anti-aging and neuroprotective studies and propose their potential applications in food and pharmaceutical industries.

43

\section{PROTECTIVE EFFECT OF A STURGEON EGG HOMOGENATE MARINE COMPOUND BUT NOT OF EPA/DHA ON ARTERIAL ULTRASTRUCTURE IN SPONTANEOUSLY HYPERTENSIVE RATS}

Bertuccelli G, Marotta F, Illuzzi N, Nagpal R, Zerbinati N, Naito Y, Mohania D, Milazzo M, Sapienza C, Italia A, Tomella C, Catanzaro $R$ ReGenera Research Group for Aging Intervention, Milan, Italy 
It has been reported that with hypertension there is an increase in lipid peroxidation products of erythrocytic membranes of humans and of aortas of spontaneously hypertensive rats (SHR). Indeed, SHR are considered to be a genetic model of hypertension mimicking idiopathic hypertension in humans. Given the uniqueness of the composition of sturgeon eggs as compared to other marine species as for PUFA and phospholipid moieties and in consideration of our recent work proving the efficacy of a controlled caviar-derived homogenate (LD-1227, Caviarlieri, LabDom, Switzerland) as an antioxidant/anti-inflammatory modulator in the brain, a typical lipidic mielieu, we tested this compound on the ultrastructure of the SHR aorta. Sixty twomonth-old SHR with initial body weights varying from 140 to $160 \mathrm{~g}$ were randomly divided into three groups of twenty-five rats each. The first group (control) was fed only rat chow; a second with 10mg EPA/DHA-added food and a third was given $10 \mathrm{mg}$ of LD-1227 added to the food for 18 weeks. Blood pressures in the carotid and femoral arteries were measured by direct cannulation using a Grass polygraph with transducers. At the end of 4 months, the SHR were anaesthetized by intraperitoneal injections of nembutal. The aorta of each SHR was dissected free from surrounding tissues. Specimens from different parts of the aortic arch were used for: measurement of thickened intima area in which areas of nine sections of the aortas of each rat were measured, averaged and taken as the value for one aorta. The data was then converted to its actual area by using a stage micrometer. Part of these specimens were also processed to postfixation with $\mathrm{OSO}^{4}$, embedding with Epon 182, double staining with uranyl acetate and lead citrate, and electron microscopic examination with JEM 7A. Both examinations were scored using a computer-assisted image-analyser. Systolic blood pressure in SHR was significantly higher than in normal Wistar rats and was not affected by LD-1227 treatment during the observation period. In the aorta of control SHR, the most prominent change was an expansion of the subendothelial space and infiltration of the mononuclear leukocytes into the intima whereas these findings were significantly reduced in LD-1227-fed rats and at lesser extent in EPA/DHA-group ( $p<0.05$ vs control and EPA/DHAgroup). TEM analysis showed structural alteration of endothelial cells with severe subcellular injury (damaged mitochondria, several vacuoles and electronlucent chromatin). Unlike EPA_DHA-group, LD-1227-treated rats displayed a significant reduction of such changes $(p<0.05)$. While detailed biochemical research is needed, these preliminary data suggest that LD-1227 has stronger arterial protective properties than EPA/DHA and it is worth further longer term studies in view of a preventive strategy medical approach.

Keywords: sturgeon egg preparation, arterial ultrastructure, EPA/DHA, Caviarlieri

\section{4}

\section{MONKEYING AROUND WITH CALORIE RESTRICTION: IS A CALORIE A CALORIE?}

Ingram DK, Roth GS, de Cabo R, Mattison JA Pennington Biomedical Research Center, Louisiana State University System, Baton Rouge, LA

Dietary caloric restriction (CR) is the only intervention repeatedly demonstrated to retard the onset and incidence of age-related diseases, maintain function, and extend both lifespan and healthspan in mammals. In 70 years of study, such beneficial effects have been demonstrated in rodents and lower animals, but prior to 1987, had never been examined in primates. To determine whether $\mathrm{CR}$ might eventually be applied to humans, the NIA initiated a study of CR and aging in nonhuman primates. After 25 years, approximately 150 rhesus monkeys (Macaca mulatta) have been involved in the study, mostly rhesus monkeys aged across their respective lifespans at the time of initiation. Control monkeys received two meals per day of a standardized, natural ingredient diet sufficient to attain apparent satiety, while the CR group received 30\% less, adjusted for age and body weight. The diet is supplemented with extra micronutrients such that the only substantive variable is the amount of calories consumed. Results to date indicate that CR animals are healthier than fully-fed counterparts based on reduced incidence of various chronic diseases, exhibit significantly better indices of predisposition to disease (such as lower insulin levels and greater insulin sensitivity, reduced blood lipids and pressure, and elevated HDL), and appear to age at a slower rate, based on a number of hormonal and functional indices, including behavioral performance. In addition, CR rhesus monkeys that were juveniles at the onset of the study showed delayed skeletal and sexual maturation, and CR groups have lower body temperatures than controls. Regarding the health of older monkeys, our results indicate several significant beneficial effects and few negative effects. However, a recent analysis of current mortality data indicated no evidence of improved survival when CR was initiated at younger or older ages, which contrasted with results reported from the University of Wisconsin study of CR in rhesus monkeys. How differences in dietary regime might account for the differences in results will be discussed.

Keywords: calorie restriction, primate, aging, NIA

45

\section{CHARACTERIZATION OF DECELLULARIZED THYMUS SCAFFOLDS FOR USE IN ENGINEERING THYMUS TISSUE}

Jackson JD, Ryu SW, Kim N, Busse K, Ju YM, Albanna M, Soker S, Yoo JJ

Wake Forest School of Medicine Institute for Regenerative Medicine Winston Salem, NC

The thymus is a primary lymphoid organ that is responsible for the maturation of T lymphocytes. During aging, the thymus undergoes a process of involution. This results in a decrease in $\mathrm{T}$ cell output from the thymus as well as a change in the distribution of T cells in the periphery. To overcome these problems of aging, we are developing an engineered thymus that may provide a method to increase the number of naive mature $\mathrm{T}$ cells and to enhance immune responses to pathogens. The goal of this project is to engineer a thymus by developing decellularization protocols to produce natural scaffolds having the potential to support the production of a functional thymus. Thymi from mouse and pig were treated with $2 \%$ Triton-X 100 and $0.1 \%$ ammonium hydroxide to remove all cellular components of the organ. The resultant decellularized scaffolds were characterized. The decellularization procedure resulted in the elimination of all cells in both mouse and pig scaffolds. Masson's trichrome staining of the scaffolds showed a decrease in collagen in the mouse scaffolds compared to the pig scaffolds, suggesting more damage to the mouse scaffold during the decellularization procedure. In addition, staining of the scaffolds using Alcian Blue indicated a reduction in glycosaminoglycans in both the mouse and pig scaffolds after the decellularization procedure. Acid extraction of the decellularized porcine thymus scaffold contained activity that increased the proliferation of thymus epithelial cells. The results indicated that the decellularization procedure 
eliminated the cells in the thymi but caused damage to the scaffolds. The porcine scaffolds appeared to sustain less damage than the mouse scaffolds and retained activity that increased epithelial cell proliferation. The ability to induce epithelial cell proliferation will be important for epithelial recellularization of the scaffolds to produce an engineered thymus. In summary, the decellularization procedure eliminated the cellular components of the thymus but caused damage to the scaffolds, resulting in decreased collagen and proteoglycan content. However, some of the activity that induced epithelial proliferation remained on the porcine scaffolds. The results show that decellularized thymus scaffolds have the potential to be used as the foundation for tissue engineering of the thymus.

Keywords: thymus, tissue engineering, scaffold

46

\section{TELOMERE DYSFUNCTION AND STEM CELL AGING}

Ju Z

Institute of Aging Research, Hangzhou Normal University

Current evidence suggest that the functional decline of adult stem cells contributes to the impaired organ homeostasis and functionality during aging. The mechanisms of stem cell aging are still poorly understood. Telomere dysfunction represent one of the molecular mechanisms limiting adult stem cell function by triggering both cell intrinsic checkpoints and cell extrinsic alterations. Deletion of DNA damage checkpoints induced by telomere dysfunction can rejuvenate the aging stem cells and improve organ function in 3rd generation telomerase knockout mice (G3Terc $\left.{ }^{-/}\right)$. However, the engrafted wild-type HSCs in G3Terc ${ }^{-/-}$mice showed abnormal hematopoiesis, which was associated with environmental defect induced by telomere dysfunctional in an age dependent manner. Further analysis revealed that telomere dysfunction induced alteration of systemic environmental factors contribute to the impaired lymphopoiesis and the decline stem cell functionality. By using a genetically modified mouse model, we tested the hypothesis that whether HSC transplantation could slow down the telomere-driven aging in the setting of ameliorate environmental defects. We found that wild-type HSC engrafted in $\mathrm{G}^{2} \mathrm{Terc}^{-/-}$ knockout mouse showed impaired hematopoiesis, whereas HSC engrafted in Exo-1 ${ }^{-/-}$, G3Terc ${ }^{-/-}$double knockout mouse showed normal hematopoiesis. Further analysis showed an increased survival in Exo-1 ${ }^{-/-}$, G3Terc ${ }^{-/-}$double knockout mouse after wild-type HSC transplantation compared to those untreated mice, indicating that transplantation of wild-type HSC could rescue the survival of telomere dysfunctional mice in the setting of ameliorated environmental defects. We also utilized iPS and ntES approaches to rejuvenate the somatic cells from aged telomere dysfunctional mice. Our data showed that ntES cells exhibited remarkable elongation of telomere length and improved mitochondrial function, associated with a better self-renewal ability and developmental potential compared to iPS cells derived from G3Terc ${ }^{-/-}$somatic cells.

Keywords: telomere, aging, stem cell

\section{7}

\section{IS THERE ANY BENEFICIAL EFFECT OF S. BOULARDI LYSATES ON NK CELL ACTIVITY IN OTHERWISE HEALTHY BUT IMMUNOCOMPROMISED ELDERLY SUBJECTS?}

Kantah MK, Takadanohara H, Marotta F, Illuzzi N, Naito Y, Celep G, Milazzo M, Sapienza C, Tomella C, Kobayashi R, Catanzaro $R$ Suheiro Chem-Tech Center
The aim of the present study was to test the effect of KC-1317, a symbiotic mixture containing microbial lysate (Saccharomyces boulardii lysate in a cranberry, colostrum-derived lactoferrin, fragaria and lactose mixture, Named, Italy) in healthy subjects with decreased NK activity. The criteria for selection were the admission criteria for immunogerontological studies (SENIEUR protocol), a World Health Organization (WHO) performance status (PS) of grade 1, 2 or 3, and informed consent. Subjects who showed NK cell activity $<10 \%$ (bottom $30 \%$ of population) were selected for the trial (39 out of the of the initially 126 screened). It was seen that average NK cell activity of the entire population was $12.4 \pm 0.54 \%$ while the average activity of selected volunteers was $6.4 \pm 0.41 \%$. Subjects were randomly distributed in two groups which were matched as for baseline activity of NK cell of $(6.2-7.4 \%)$ and for dietary and life-style questionnaire answers. (A- $100 \mathrm{ml}$ of fruit juice as placebo: B $-90 \mathrm{ml}$ of Placebo with KC$1131710 \mathrm{ml})$. The subjects were asked to consume the supplement twice daily, in the morning and evening for 2 months. Peripheral blood samples were withdrawn at two different time points during the trial (once during selection and another after completion of trial-week. Isolation of Lymphocytes. Peripheral blood mononuclear cells were isolated by histopaque density gradient centrifugation. NK cell activity was measured from the blood at the indicated time points without further priming. NK Cell Activity. K562 cell line will be continuously maintained in RPMI medium in presence of $10 \%$ Fetal Calf Serum. They will be sub cultured every $3-4$ days and were incubated at $37^{\circ} \mathrm{C} \& 5 \% \mathrm{CO} 2$ atmosphere. Post incubation, cells were centrifuged, washed with PBS and suspended in $100 \mathrm{ml}$ PBS. Fluorescence measurements

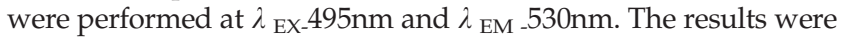
expressed as (\%) lytic activity of NK cells. As compared to placebo-treated group, B group showed an overall significant increase of NK activity with an activity increased to $12.6 \pm 1.3 \%$ $(\mathrm{p}<0.01)$. In particular, 9 subjects $(23.5 \%), 11$ subjects $(28 \%)$ and 19 (subjects $48.5 \%$ ) showed a mild ( $<100 \%$ increase in NK cell activity), moderate (100-200\% increase in NK cell activity) and intense response ( $>200 \%$ increase in NK cell activity) response, respectively. Although these data need larger and longer-term studies together with correlations with several others health status markers, they also offer a promising avenue to be pursued within a wider global health strategy by providing a safe dietary supplement enhancing innate cellular immune function.

Keywords: Saccharomyces boulardii lysate, NK cell activity, KC-11317

48

\section{AGING AND EXTERNAL SIGNALING}

\section{Khalyavkin AV, Krutko VN}

Institute of Biochemical Physics of RAS \& Institute for Systems Analysis of RAS, Moscow, Russia

Researchers working in the area of aging found numerous manifestations of this process at the molecular biological level including DNA and protein damage, accumulation of metabolic by-products, lipids peroxidation, cross-linking between macromolecules, non-enzymatic glycosylation, anti-oxidant/pro-oxidant misbalance, rising of pro-inflammatory cytokines etc., resulting in an increase in proportion of cells in growth arrest, reduction of the rate of information processing, decreasing metabolic rate, and rates of other processes characterizing dynamic aspects of organism's interaction with environment. Such variability in manifestation of senescence precludes development of systemic view on its primary causes and subsequent compensatory adaptation, and does not 
allow for developing insights and ideas concerning approaches capable of postponing aging or reducing organisms' aging rate. It turns out that damage production and damage elimination processes which misbalance results in the aging process can in a large extent be regulated by external signals and can be reduced up to zero level. Many experimental interventions and some indirect findings indicate on the reality to evade senescence by external stimulation only, without any genome modification (e.g. useful mutations, overexpression etc.) and can prompt us the ways to full aging retardation and even its reversion. We suggest that such reversion of aging should be an ad hoc variant of so-called negative senescence (Vaupel et al. 2004), when potentially non-senescent organism which was aged in non-supportive conditions starts to restore lost status quo via positive external influences.

Keywords: environmental influences, origin of aging, retardation of senescence

\section{9}

\section{NON-SENESCENCE SEEMS FEASIBLE. BUT ARE WE READY NOW FOR SUCH POSSIBILITY?}

Khalyavkin $A V$

Institute of Biochemical Physics of RAS \& Institute for Systems

Analysis of RAS, Moscow, Russia

Are we ready to be non-senescent now? We may say 'Yes' if it is a part of the scientific task. But we must say 'No' in a case if it will be a global social project. It is so since at present our society is too heterogeneous, too imperfect and too inconsistent for the realization of this possibility. Therefore the overcoming of social imperfectness is a top-priority challenge of our days. While antiaging researchers have made considerable advances in recent years, anti-aging thinking, as a formal discipline, has yet to enter the mainstream. I believe that this is partially a consequence of insufficient attention to and an underestimating of the future social issues - both the negative and positive. But what are the biological bases of our confidence in feasibility of non-senescence? The limitless replicative potential which somatic stem-like cells are thought to possess is clearly revealed by means of in vitro restoration of the stem cell supporting microenvironment, via removing differentiating stimuli from the cell culture, or by adding differentiating inhibitors to cultural medium, etc. We also know that the control system of a potentially non-senescent organism is able to sustain a physiological regimen of complete selfmaintenance not in any circumstances but only within a certain range of changes in the total external conditions known as "environmental pressure". Outside the zone of adequate environmental pressure self-maintenance will be incomplete. The resistance of organism will start to diminish, and it will begin to age. Even ageless Hydras start to experience a Gompertzian (exponential) aging in such conditions. The patterns of human mortality are also compatible with this view. But certainly we must have a reliable road map for the future harmonic society long before possible wide world realization of ageless individuals.

Keywords: Non-senescence, Social issues, Global road map

\section{0}

\section{WHETHER IS PHENOPTOSIS AN INEVITABLE PROGRAMMED AGING OR IT CAN BE AVOIDED?}

Khalyavkin $A V$

Institute of Biochemical Physics of RAS \& Institute for Systems

Analysis of RAS, Moscow, Russia
The senescence is a complicated and yet not fully comprehended process. Currently there is a lot of data contradicting to the theories of occurrence of aging due to accumulation of various damages. For instance, there has been partial or complete reversibility of symptoms indicating the senescence of organism components as a result of creating essential conditions for their functioning (relevant microenvironment, humoral background, etc). Following for V. Skulachev the programmed senescence and death of an organism can be defined by the term phenoptosis, similar to the term apoptosis for the programmed death of a cell. Beginning from A. Weismann, the necessity of this phenomenon is explained by the need for turnover of population according to ecological and evolutionary demands. Species-specific life span, age-dependent changes in the pattern of genome expression, etc. are consistent to the conception of phenoptosis. However, it became clear that the intra-specific rate of aging may vary over of a wide range, depending on the living conditions. This means that the "senescence program" is not strictly preset, but is very flexible and adjusts the organism in response to concrete conditions of the habitat. Moreover, there are findings indicating that in severe habitat conditions the senescence program can be completely switched off (Khaliavkin, 2001; Seymour, Doncaster, 2007). There is no further need in this program because of the high death rate due to external reasons (high extrinsic mortality), which in turn provides for faster population turnover. Aforementioned approaches mean that the stochastic mechanisms of senescence are likely a secondary. However, the genetically driven phenoptosis should not be obligatory permanent. It may emerge due to inducible quasi-program of senescence, which initiates under some influences of the concrete environment. These influences expel the organism out of the zone of dynamic stability. The senescence pace is dependent on the magnitude of deviation of vital activity parameters from the stability zone limits.

Keywords: phenoptosis, quasi-programmed aging, avoidable senescence, dynamic stability

\section{1}

\section{LEGAL PROBLEMS OF REGISTERING SUBSTANCES AND THERAPIES TO CURE AGING}

\section{Khaltourina D}

Council for Public Health and Demography, Russia

A number of compounds are known to have some anti-aging effects, reducing the rate of aging or extending the lifespan in animals and probably in humans. At the same time, these drugs are not widely marketed to the general public as cures against aging, which probably causes unnecessary losses of healthy life years for many people.

Health experts blame the international legal framework, which only allows registering drugs against certain diseases. This refers to Food and Drug Administration in the United States, and other national agencies which all follow the World Health Organization's guidelines for drug registration. As a result, some geroprotective substances are registered as either drugs against certain diseases (for example, diabetes) or food supplements.

One of the reasons named for this situation is that aging is not in the official list of diseases in the International Classification of Diseases. At the same time, there is a number of conditions which are not considered as diseases, like pregnancy, but there are medicines which are prescribed for these conditions. 
Another problem is that most anti-aging drugs and therapies are supposed to have preventive rather than treating effects for a broad spectrum of diseases, and it would take long and expensive clinical trials to justify their beneficial effects. Registering geroprotective substances as medicines for more than one disease seems to be a workable solution. However, geroprotective effects can be more pronounced for some conditions, and much smaller for others, which would make it difficult for them to compete with drugs targeting specific diseases.

Another option is to promote the inclusion of aging into the official disease classifications which would require coordinated advocacy efforts at the global level. Alternatively, conditional registration of new drugs and therapies to cure and prevent aging should be developed and promoted. In any case, a system for testing drugs and therapies to cure aging effectiveness should be established.

In sum, the most promising strategies to ensure registration of new drugs and therapies to cure aging are:

- advocacy for acknowledging age related conditions like sarcopenia as treatable diseases;

- international lobbying to acknowledge aging as a treatable and partly preventable condition;

- promoting legal framework for conditional registration for the drugs and therapies to cure aging.

Keywords: legislation, advocacy

52

\section{AGE-ASSOCIATED CHANGES IN WALKING PARAMETERS OF JAPANESE ADULT WOMEN}

Kikkawa K, Okada H, Oishi R

Faculty of Informatics and Engineering, The University of ElectroCommunications

Earlier, we proposed an age-predictive equation, based on several walking parameters screening in male subjects $50-80$ years of age. However, with this equation, the predicted age is deviated from the actual age that was statistically justified in a wider range. With this in mind, the current study was undertaken to clarify the parameter of age-associated changes in walking movements and the occurrence age.

A two-dimensional walking movement analysis was conducted on women grouped into various ages. The participants of this study were 177 healthy women aged from 19-82 years. They were categorized into eight age groups, from the younger ones according to the decade of their age, under 20 (F20), 21-30 (F30) ..., 51-60 (F60), and for beyond 60 every five years, 61-65 (F65) ..., 71-75 (F75).

An analysis of covariance was adopted using gait velocity as covariate, together with the age groups (F20, F30, F40, F50, F60, $\mathrm{F} 65, \mathrm{~F} 70, \mathrm{~F} 75)$ as a fixed factor, were used to examine the differences in each group (The performance-descriptors applied were step length, step frequency, velocity, and each phase time; Kinematics as ankle angle, knee angle, and hip angle; and Kinetics as peak joint torque power and positive/negative work of each joint).

The results clarified the following two points:

1. The analysis of the gait velocity indicated that, the step length decreases and the step frequency increases with ageing. (In addition, the gait length correlated with the height.)
2. Age-associated changes in the gait patterns showed an increased step frequency but with a decreased step length for the 50's. For the groups of 60 's and beyond, with a more flexed knee joint angle to avoid vertical motion was found. An increased positive work of the hip joints and a more flexed hip angle could be observed in the 70's and beyond.

We conclude that age-associated changes in walking movement occur due to the essential ageing effect in the walking, rather than to the differences in the physique and the decrease of the gait velocity. In addition, it is suggested that the changes in walking movements can occur at any age groups.

Keywords: ANCOVA, walking, kinematics, kinetics, Japanese

\section{3}

\section{EFFICIENCY OF CELLULAR DIVISION WHEN CREATING SMALL POCKETS OF ELECTRIC CURRENT ALONG THE WALLS OF CELLS}

\author{
Kletetschka G, Zila V, Klimova L \\ Charles University in Prague, Faculty of Science, Czech Republic
}

There is likely a relation between the electromagnetic field and cancer growth. Magnetic field of $50 \mathrm{~Hz}$ and 100 microtesla enhanced the tumor development and growth in the breast cancer in female Sprague Dawley rats (Thun-Battersby et al., Cancer Res., 59: 3627-3633, 1999). It has been hypothesized that the increased breast cancer in industrial societies is related to greater exposure to power-frequency electric and magnetic fields (EMF). EMF is thought to reduce the circulating level of melatonin, increasing the estrogen levels and stimulate the turnover of epithelial stem cells that may possibly lead to malignant transformation (Graham et al., Environ Health Perspect., 109(5): 501-507, 2001). The consensus has not been found and therefore we utilized three different cell cultures, bacteria Escherichia coli, murine 3T6 fibroblasts and murine embryonic stem cells (mES) for exposure with pulses of various magnetic field peak intensities from $1 \mathrm{mT}$ to $11,000 \mathrm{mT}$, using ASC Magnetizer Model IM10-30. Peak lasted about 1-3 seconds and was responsible for inducing currents within the cells that were decaying with time due to ohmic losses.

The culture of E. coli was experiencing magnetic pulse of at least $11,000 \mathrm{mT}$. Cells were exposed 1- or 10-times with $11,000 \mathrm{mT}$, and then plated on Petri dishes with agar for about 5 hours. As a control non-exposed cells were plated. The exposition of E. coli to magnetic pulse of 11,000 mT did not result in cell death, Magnetic pulses dramatically enhanced multiplication of bacteria in comparison to our control samples. Cells exposed to just one magnetic pulse, increased their amount by $370 \%$, while cells exposed to magnetic pulse 10-times increased their amount by $260 \%$. Exposure 10 times was probably not as beneficial but overall culture still outperformed the controls. Murine 3T6 fibroblasts were exposed with peak field intensities of $0.8 \mathrm{mT}$, $1.2 \mathrm{mT}, 13 \mathrm{mT}, 108 \mathrm{mT}, 1370 \mathrm{mT}$, and $9408 \mathrm{mT}$. Murine ES cells were used for the field exposure of $13 \mathrm{mT}, 108 \mathrm{mT}, 1370 \mathrm{mT}$, and $9408 \mathrm{mT}$. After exposition, 3T6 fibroblasts and ES cells were seeded to wells of 6-well plates and cultivated in culture medium for 48 or 96 hours respectively. For both cell types, the increase in EMF intensity did not result to cell death. Pulse of $108 \mathrm{mT}$ increased numbers of 3T6 fibroblasts in culture by $40 \%$, while after exposition to other intensities amounts of cells were comparable with the control. Exposition of ES cells to any of tested magnetic field intensities did not affect their ability to 
grow and amounts of cells at time of counting was always comparable with the control.

These preliminary experiments indicate resistance of E. coli cells as well as mammalian murine fibroblasts or embryonic stem cells to exposition to high magnetic field intensities. Observation of enhancement of multiplication of E. coli was in contrast to insignificant effect of magnetic pulses on growing of murine 3T6 or ES cells, suggesting that effect of high magnetic field intensities might be cell type dependent and might differ between prokaryotic and eukaryotic cells.

Keywords: murine embryonic stem cells, murine 3T6 fibroblasts cells, Escherichia coli, EMF, magnetic field

\section{4}

\section{REIMAGINE AGING}

Kope $M$

SENS Research Foundation

We face a global crisis in the rising cost of healthcare for an aging population. Worldwide we spend trillions of dollars annually on healthcare for seniors. Yet heart disease, long the greatest of the UK and US health problems, has recently become the world's biggest killer as well. There's an immense amount of research being poured into the development of treatments for age-related disease, gerontological research organizations are increasingly focusing on translational results, and the word 'healthspan' is now in common use. Now, we need to refocus some of this growing energy into attacking not only the pathologies of aging, but their underlying causes. We'll review SRF's scientific, educational, and outreach-based efforts to achieve that refocusing; reimagining research paradigms, reimagining our ability to cure age-related disease, and reimagining aging.

Keywords: aging, healthcare, healthspan, gerontology, policy

\section{IS TRANSCRIPTION THE KEY TO THE SELECTION ADVANTAGE OF MITOCHONDRIAL DELETION MUTANTS?}

Kowald A, Kirkwood TBL

University of Newcastle Institute for Ageing and Health Campus for Ageing and Vitality Newcastle upon Tyne NE4 5PL United Kingdom

The mitochondrial theory of ageing is one of the most popular mechanistic theories of the ageing process. Mitochondrial energy production is of central importance to the health and proper functioning of cells and single cell studies have shown that mtDNA deletion mutants accumulate in a clonal fashion in various mammalian species, overtaking the cellular population of wild-type mtDNAs. An understanding of the biochemical process, which leads to this clonal expansion would be of great importance, but unfortunately the existing ideas such as random drift, size advantage of deleted genomes or survival of the slowest are incompatible with experimental observations. We present here a new idea that is based on the peculiar connection between transcription and replication of metazoan mtDNA. Computer simulations of this new mechanism show that it is compatible with a large range of lifespans and the observed low degree of heteroplasmy. Furthermore, bioinformatical studies of single cell deletion spectra show exactly the type of distribution that would be predicted by our hypothesis.

\section{ESTETROL, MOLECULAR CHAPERONES AND THE EPIGENETICS OF LONGEVITY AND CANCER RESISTANCE}

Krøll J

Hafnia Unit of Biogerontology

The lifespan in mammals varies from a few years in rodents to more than 100 years in humans. The rapid evolution of longevity in mammals suggests the involvement of relatively few genes, as also the inherent immortality of embryonic stem cells in vitro, suggests that replicative senescence - as possibly organismal aging - are epigenetic phenomena.

That the molecular chaperones are probably involved in the evolution of longevity; appears from the observation that the chaperones are essential for the process of cellular immortalization, also the level of expression the chaperones correlate to the longevity of species as well as to the lifespan of differentiated cells in the organism.

Weakening of the chaperone defence with age probably contributes to the phenotype of aging.

The present review presents evidence that the Estrogen: Estetrol promotes the expression of the molecular chaperones and adds to species-and cellular longevity and cancer resistance

Keywords: Estetrol, Chaperones, longevity, cancer resistence

57

\section{INTERNATIONAL FUTURES: IMPACT OF DECELERATED OR ABOLISHED AGING ON HUMAN DEMOGRAPHIC CHANGE}

Kuhn R

Josef Korbel School of International Studies, University of Denver

In spite of rapid and even accelerating progress in extending longevity and delaying the effects of aging, few existing forecasts have explored the potential consequences of dramatic reductions in mortality on demographic and socioeconomic outcomes. This presentation describes the early results from a forecast of extreme longevity scenarios using an integrated, country-level, long-range International Futures (IFs) forecasting system. We describe the extensions we have made to the IFs, the scenario-building process, and preliminary findings.

We compare three longevity scenarios: i) a base-case conforming to typical UN/OECD expectations of a maximum population-level life expectancy in 2100 of about 94 with a persistent burden of morbidity; ii) a delayed senescence scenario in which the existing mortality curves are pushed back by 20 years, yielding a maximum life expectancy of about 110 and an accumulating burden of morbidity at oldest ages; and iii) a regenerative scenario in which agespecific mortality and morbidity rates approach zero for all ages. We also explore uncertainty in the pace of cross-country diffusion of longevity interventions. Finally, we address the potential consequences of regenerative medicine for restored fecundity; we develop hypothetical fertility functions and model their impacts.

We will explore each scenario in two steps. We first describe the world that might emerge if the first-order "mechanical" effects of declining mortality, declining morbidity, and rising fecundity were not met with policy change. We next explore some of the policy changes that might be needed to accommodate a new human physiological regime.

Keywords: International Futures, demographics, mortality, fertility, policy 
58

\section{DIETARY AND NUTRITIONAL MANIPULATION OF THE NUCLEAR TRANSCRIPTION FACTORS, PPAR'S AND SREBP'S, AS A TOOL FOR REVERSING THE PRIMARY DISEASES OF PREMATURE DEATH AND AGING}

Kurtak K

Grossman Wellness Institute

2.1 billion years of evolution has equipped us with a biochemical pathway that has the power to arrest, and in some cases reverse, the primary disease etiologies of aging and premature death in the developed world. Activation of the PPAR pathway arrests inflammatory signaling throughout the body, reverses damage to tissues, reverses insulin resistance and can even dissolve betaamyloid plaque in the brain. It has played a critical role in the evolution of the metazoans and the successful migration of humans to all corners of the Earth. For two decades, various pharmaceuticals have been designed to activate the PPAR pathway but have consistently fallen short of expectations. There is nothing wrong with these drugs. The problem has been the standard "healthy" diet creating mixed signals that render the drugs ineffective. This talk explores the ongoing dance between the two primary nuclear receptors that mediate gene regulation of fatty acids. It discusses their interaction with sirtuins and telomerase, optimization of their obligate heterodimers, and why manipulation of dietary and nutritional factors are the most effective means of activation. These are effective tools that can be implemented NOW to slow, and in some cases reverse, the diseases of aging.

Keywords: Diseases of aging, Nuclear Transcription Factors, PPAR, SREBP, Inflammation

59

\section{ECTOPIC ORGANOGENESIS IN LYMPH NODE}

Komori J, Boone L, DeWard A, Hoppo T, Rao A, Paulo F, Lagasse E McGowan Institute for Regenerative Medicine

The shortage of organs is today a major health crisis. We are living longer and as we aged, our organs tend to fail more. Currently, there are not enough organs to go around and, in fact, in the last ten years the number of patients requiring organs has doubled when at the same time, the actual number of transplant has barely gone up.

One possible solution for this public health crisis is to grow tissues and organs in the laboratory for their eventual application in patients. While this approach has revealed encouraging results, major challenges for growing organs, like blood supply and vascularization of the tissues, have hampered the effort.

My lab has tested the concept that certain locations in the body may be amenable to in vivo tissue and organ regeneration. We hypothesize that the lymph node (LN) in particular offers a rich environment that is well suited for this task. Our rational was based on several observations. The LN function is an in vivo bioreactor to promote lymphocyte survival and expansion in response to an antigen. However, the $\mathrm{LN}$ can also serve as an inviting host to more than just lymphocytes. After a series of genetic changes, selected cancer cells will often migrate away from the primary tumor and colonize in the LN to form a new tumor mass. Why is the LN environment so accommodating to lymphocytes as well as to a variety of tumor cells? And could LNs provide a suitable environment to maintain survival and expansion of normal cells other than lymphocytes?
Using three different organs, liver, thymus and pancreas, we provide the first report and demonstrated the proof-of-concept that the LN provides a hospitable environment for normal epithelial cell engraftment and function. Our approach reveals an important technical methodology to consider for future ectopic tissue and organ regeneration.

Keywords: Organogenesis, Lymph node, Vasculature, Liver, Thymus

60

\section{SODIUM-POTASSIUM ATPASE MODULATION FOR AGING RELATED DISEASE}

Larrick JW, Wages J, Sharma V, Wang H, Wei X, Wang J, Mendelsohn A, Wright SC

Panorama Research Institute

An urgent need exists to develop novel therapies for cardiac failure. The $\mathrm{Na}+/ \mathrm{K}+$ ATPase (NKA) catalyzes active transport of $\mathrm{Na}+$ and $\mathrm{K}+$ ions across the cardiomyocyte plasma membrane. Cardiac glycosides are classically thought to have a positive inotropic effect by inhibiting NKA which increases intracellular $\mathrm{Na}+$ which is exchanged with $\mathrm{Ca}++$ to augment contractility. Although this mechanism is undoubtedly more complicated (Nishio, 2002), recent work identified an activation site on NKA. An affinity purified antiserum specific for this extracellular site markedly augments NKA catalytic activity both in vitro and in vivo in rodents (Zheng, 2011). The augmented NKA activity is correlated with a positive cardiotonic action and perhaps most importantly activation of cardioprotective pathways in vivo. Spontaneously hypertensive heart failure rats (SHHF) develop a severe, lethal hypertensive cardiomyopathy. High titer anti-NKA antibodies elicited in SHHF by active immunization completely prevented the lethal heart failure (HF) of these animals with no noticeable adverse effects over a period of 30 weeks. Based on these initial proof-of principle studies, we utilized our human Fab phagemid library to identify HuNKA, a high affinity anti-NKA human monoclonal antibody. Our preliminary in vitro studies indicate that HuNKA not only exhibits a positive cardiotonic effect but initiates cardioprotection via activation of extracellular signal-regulated kinase $1 / 2$ and phosphoinositide 3-kinase/Akt pathways. Based on these activities we are optimistic that HuNKA will provide a novel immunotherapeutic approach for heart failure.

Keywords: Therapeutic antibody, $\mathrm{Na}+/ \mathrm{K}+$ ATPase, Heart failure

61

\section{THREE-PATHWAY MODEL OF MAMMALIAN LONGEVITY}

Lehmann G, Muradian KhK, Fraifeld VE

The Shraga Segal Department of Microbiology, Immunology, and Genetics, Center for Multidisciplinary Research on Aging, Ben-Gurion University of the Negev, Beer Sheva, Israel

Why do species differ in lifespan and what are the determinants of their longevity? Understanding the main factors that determine variation in species longevity may provide a clue into the leading mechanisms of aging and-what is even more important-outline the key targets for longevity-promoting interventions. We have previously proposed a "two-pathway longevity model" which highlights 1) the importance of metabolic 
stability associated with lower resting metabolic rate (RMR) and/ or higher body mass (BM), and 2) mtDNA thermodynamic stability ensured by higher GC content. Including the additional factors of body temperature $\left(\mathrm{T}_{\mathrm{b}}\right)$ and maximum metabolic rate (MMR), further enhances predictive power of the model and may better explain the cases of exceptional lifespan among mammals. The new model suggests that reducing the RMR and/or increasing the MMR and lowering $\mathrm{T}_{\mathrm{b}}$ could be potential strategies for lifespan extension. Although lowering $\mathrm{T}_{\mathrm{b}}$ and/or modulating the metabolic rate are not trivial tasks, they are technically much more feasible than genetic interventions with their hardly predictable consequences. Another important point is that these strategies are not "foreign" for endothermic organisms since they are based on preexisting, evolutionary conserved programs for adjusted resetting of homeostatic systems.

Keywords: Mammalian longevity, Metabolic scope, Body temperature, mtDNA, models

\section{2}

\section{LATENCY-ASSOCIATED DEGRADATION OF THE MRP1 DRUG TRANSPORTER PRESENTS A NOVEL THERAPEUTIC TARGET FOR LATENT HUMAN CYTOMEGALOVIRUS INFECTION}

Weekes MP, Tan S, Poole E, Talbot S, Antrobus R, Smith DL, Gygi $S P$, Sinclair JH, Lehner PJ

Cambridge Institute for Medical Research, University of Cambridge, Hills Road, Cambridge, CB2 OXY, UK

Reactivation of latent human cytomegalovirus (HCMV) infection following transplantation is associated with high morbidity and mortality. Strategies to prevent disease will necessarily require approaches to deplete latently-infected cells. In vivo, myeloid cells and their progenitors are an important site of HCMV latency, whose establishment and/or maintenance requires expression of the viral UL138 gene.

To address how UL138 affects host cell surface receptor expression during latent HCMV infection, we developed 'plasma membrane profiling' (PMP), a proteomic technique that employs SILAC-based differential analysis to compare the expression of plasma membrane proteins in the presence and absence of UL138 in undifferentiated myeloid cells. Of the 592 plasma membrane proteins isolated from the monocytic cell line THP-1 only three were reproducibly affected more than two-fold.

We found a dramatic UL138-mediated loss of the cell surface transporter Multidrug Resistance-associated Protein-1 (MRP1), which usually functions to export a range of endogenous substrates and cytotoxic drugs. UL138-expressing and productively HCMVinfected cells exhibited a marked reduction in export of MRP1specific substrates such as the fluorescent reporter SNARF-1 and the endogenous leukotriene LTC4, suggesting that cytotoxic MRP1 substrates might be employed to kill cells infected with HCMV.

We confirmed latency-associated loss of MRP1 in an experimental model of latent HCMV infection, and showed that accumulation of the cytotoxic MRP1 substrate vincristine dramatically reduced levels of reactivated virus after myeloid cell differentiation, a well-established signal for virus reactivation. Thus using the cytotoxic MRP1 substrate vincristine, we can now target HCMV-infected cells in natural latency, providing a therapeutic opportunity to purge HCMV-infected cells. The UL138-mediated loss of MRP1 provides a marker for both detecting latent HCMV infection and a therapeutic target for eliminating latently-infected cells prior to transplantation.

Keywords: CMV, latency, MRP1, reactivation
63

\section{APHERESIS OF TNF AND IL-2 DISRUPTS TUMOUR IMMUNOEVASION}

Lentz MR

Praxis Lentz, Germany

Immune suppression has long been recognized as a hallmark of patients with advanced malignancies. Tumor necrosis factor (TNF) and interleukin-2 (IL-2) are pro-inflammatory cytokines produced by activated mononuclear white blood cells (WBC) that bind to tumor cells via specific receptors and induce cell death by both oxidative stress and apoptosis. This process constitutes a major part of the normal response of the immune system against cancer cells.

Overproduction and shedding by tumor cells of high levels of two soluble receptors for TNF (sTNF-R1 and sTNF-R2) and IL-2 (sIL2-R) are believed to be a fundamental mechanism by which tumor cells locally block attack and subsequent destruction by the immune system. These soluble receptors act as competitive inhibitors affecting the interaction of TNF and the surface receptors of cancer cells. This mechanism explains why high levels of sTNF-R1 and sTNF-R2 have been negatively associated with the prognosis of tumor patients in several clinical and epidemiological investigations.

My work involves the extracorporeal removal of these inhibitors via a customized apheresis device and affinity column, with the goal of reducing local tissue inhibitor concentrations below the tumor-protective threshold. Multiple clinical trials and a recent mechanistic study have demonstrated that this treatment significantly reduces plasma concentrations of sTNF-R1, sTNF-R2, and sIL2-R receptors, and is well tolerated by patients.

Observed clinical effects are consistent with a treatmentinduced immune response against the tumor, including tumor pain with tenderness of palpable tumors, tumor swelling, palpable heat in tumor regions, and low-grade fever. Longer observation shows that metastatic patients who receive multiple treatment cycles and demonstrate clinical signs and symptoms of tumor inflammation subsequently demonstrate signs of tumor necrosis and tumor reduction, as evidenced by X-ray imaging and CT scans, suggesting that lowering plasma levels of sTNF-R1, sTNF-R2, and sIL2-R is beneficial to patients with metastatic cancer.

Keywords: TNF, IL-2, apehresis, cancer, metastatic

64

\section{OVERVIEW OF BEVERAGES WITH ANTI-AGING FUNCTIONS IN CHINESE MARKET}

Yang F, Song J, Liang M, Ma FL, Mao XL, Li HF, Huang ZB Research \& Development Centre, Infinitus (China) Company Ltd, Guangzhou 510665, China

Although aging is an irreversible process, anti-aging has been a dream in all cultures and all peoples at all times. For example, a number of traditional Chinese medicines have been used to slow the aging process. Interestingly, some of the anti-aging Chinese herbs are also used as food and can be seen in traditional beverages to promote health and prevent diseases. More interestingly, anti-aging herbal drinks may be used differently between men and women as in herbal medicine, reflecting the Chinese "Yin-Yang" philosophy. At the same time, modern studies have revealed that some dietary natural products can slow aging in 
Caenorhabditis elegans, mouse and other animal models. As a result, functional beverages containing anti-aging natural products have emerged in Chinese market in the past decade, challenging the dominance of traditional functional beverages. Here we summarize both traditional anti-aging Chinese herbs and natural dietary compounds currently used in functional beverages in China. We also aim to outline the underlying mechanisms of both types of beverages in slowing aging process, such as their effects on telomere-telomerase system, energy homeostasis and stress resistance.

65

\section{REGENERATION IN THE ADULT ORGANISM - A CURE FOR AGEING?}

Maden $M$

University of Florida

The most amazing organ regenerative ability is displayed by the Urodele amphibians such as axolotls which can regenerate brains, spinal cord, limbs, tails, heart, lower jaw and other structures. There are many reasons for studying regenerative mechanisms in these organisms and one of the most intriguing may be the relation between regeneration and ageing. How old are the cells of a regenerated organ - the same as the host animal or is age reset in a dedifferentiated multipotent stem cell? Nothing is known about this phenomenon apart from the demonstration that regenerative ability does not decline with age in Urodeles - the quality of the last regenerate is as good as the first. Even without this knowledge, the ability to induce organ regeneration in humans would have a huge impact on ageing and longevity. We have had some success at inducing regeneration in mammals by extrapolating from our understanding of axolotl regeneration, in particular concerning one developmental signaling molecule, retinoic acid. This molecule is critically required for limb development, limb regeneration, heart regeneration and when administered in excess induces the duplication of limbs. Its mechanism of action and targets in the nucleus are gradually being revealed and it interacts with other developmental signaling pathways. Most importantly we have shown that retinoic acid can induce a regenerative response in mammalian organs which cannot normally regenerate such as the lung and the spinal cord. We have therefore referred to this molecule as a regeneration-inducing molecule. Our recent studies have also revealed the role of retinoic acid in neurodegenerative diseases such as Alzheimer's disease and in a mouse model of this disease it has the remarkable effect of reducing plaque load and the levels of the toxic $\mathrm{Ab}$ peptide in the brain. Retinoic acid may thus really have life-extending properties and we anticipate that further research may also give us insights into the ageing process itself.

Keywords: ageing, regeneration, Urodeles, retinoic acid, Alzheimer's disease

66

\section{SPERMIDINE INDUCES AUTOPHAGY IN MULTIPLE SPECIES AND MIMICS CALORIE RESTRICTION}

Madeo F

Institute for Molecular Biosciences Humboldtstrasse 508010 Graz Austria

Spermidine is a natural occurring polyamine that declines continuously during aging of organisms. We found that external administration of spermidine triggers autophagy across species and prolongs lifespan of yeasts, flies and worms in a autophagy dependent manner. Moreover, spermidine extends healthsspan of mice.

We propose that the anti-aging potential of spermidine roots in epigenetic transformation of the cell and indicate its therapeutic potential for the avoidance of age associated diseases.

67

\section{THE HIDDEN PHENOMENON OF OXIDATIVE STRESS IN THE TREATMENT OF SUBCLINICAL HYPOTHYROIDISM: A PROTECTIVE NUTRACEUTICAL INTERVENTION}

Tomella C, Catanzaro R, Illuzzi N, Cabeca A, Lorenzetti, A Celep G, Milazzo M, Sapienza C, Zerbinati N, Italia A, Bertuccelli G, Marotta F ReGenera Research Group for Aging Intervention, MIlan, Italy

Thyroid hormones are known to be associated with the oxidative and antioxidative status of the organism. Subclinical hypothyroidism (SH), defined as an elevated serum thyroid stimulating hormone (TSH) level associated with serum thyroid hormone concentrations within the reference range, is found in $4-10 \%$ of individuals from Western populations. A very recent study suggested that oxidative stress in subjects with primary hypothyroidism under therapy with L-T4 might be the cause of the side effects commonly found with this treatment concluding that side effects following L-T4 therapy depend on OS and cause daily discomfort and loss of working activity in about $1 / 3$ of the days considered. Thus the aim of the present study was to test a redox-balance modulator, FPP, in association with treatment of $\mathrm{SH}$ or $\mathrm{MH}$ in terms of: clinical symptom score, oxidative stress, lipid profile and oxidized LDL, endothelial dysfunction test and gene expression involved in thyroid/mitochondrial regulation. 50 females, aged 18-55 with subclinical hypothyroidism or mild hypothryroidism, not on birth pill control and without main chronic diseases, relevant medications or major dislipidemia disorders. Patients were divided into two groups (25 each) matched as for age, overall biochemical status, dietary questionnaire-related profile and thyroid status assessment. Both groups received similar medical treatment for their hypothyroidism after a 2-week wash-out. One group was given: FPP $3 g r$ 1 sachet twice a day +1 sweet placebo drink (fruit flavour) twice a day. One group was given: Placebo (flavoured sugar) 1 sachet twice a day plus the same multivitamin drink twice a day. A matched group of normal thyroid functiona subjects was our healthy control (HC). Levels of T4, T3, TSH, serum malondialdehyde (MDA), protein carbonyl (PC) and plasma oxidized glutathione (GSSG) were measured as markers of oxidative stress. The plasma-reduced glutathione (GSH) level and the GSH/GSSG ratio were determined as markers of plasma antioxidant capacity. As compared to $\mathrm{HC}$ a significant increase of all oxidative markers was observed in $\mathrm{MH}$ subjects $(\mathrm{p}<0.05)$ while, T4 treatment brought about a further increase $(p<0.05)$. While placebo was ineffective, FPP-supplemented individuals showed a significant normalization of redox markers $(\mathrm{p}<0.01)$. It is concluded that thyroid supplementation by accelerating mitochondrial oxygen consumption may increase OS. Thus a rational redox-modulator integrative therapy is adviced, given also the long-term period these treatment are maintained. Gene regulation studies are under way.

Keywords: subclinical hypothyroidism, oxidative stress, thyroid hormonal supplement, fermented papaya preparation 
68

\section{ANTI-INFLAMMATORY AND ANTI-MUTAGENIC EFFECT OF YHK PHYTOCOMPOUND IN HEPATOCYTES: IN VIEW OF AN AGE-MANAGEMENT LIVER PROTECTING APPROACH}

Catanzaro R, Celep G, Illuzzi N, Milazzo M, Rastmanesh R, Yaduvanshi SK, He F, Solimene U, Sapienza C, Srivastava N, Marotta $F$

ReGenera Research Group for Aging Intervention, Milan, Italy

The receptor for advanced glycation end products (RAGE) is oncogenic and RAGE appears to operate mainly in pathological responses such as in diabetes, brain degenerative, inflammatory processes and cancers. Recently, it has been shown that RAGE regulates cellular proliferation in hepatocellular carcinoma (HCC) and it is overexpressed also in primary hepatocellular carcinoma (PHC) compared to surrounding para-neoplastic liver samples. Moreover, high mobility group box 1 protein (HMGB1) is widely expressed in tumor cells and acts as a proinflammatory cytokine creating an inflammation-associated cancer-prone microenvironment. The aim of the present study was to test the in vitro effect of $\mathrm{YHK}$, a nutraceutical with prior data suggesting its hepatocyte protecting role, in regulating RAGE in the proliferation of HCC cell line HepG2, as well checking also its potential modulation in the expression of the transcriptional factor NF- $\kappa$ B p65. Cell proliferation was performed with the 3-(4,5dimethylthiazol-2-yl)-2,5-diphenyltetrazolium bromide (MTT) colorimetric assay and time RT-PCR (qRT-PCR) and Western Blot were also used. Our study showed that YHK significantly reduced cellular growth in the HCC cell line HepG2 $(\mathrm{p}<0.05)$. Moreover, this phytocompound partly reduced gene expression of NF- $\kappa \mathrm{B}$ p65 (by $38 \%$ expressed as relative expression of NF- $\kappa \mathrm{B}$ p65 mRNA normalized to $\beta$-actin expression, $\mathrm{p}<0.05$ ) as well as RAGE ligand and cellular proliferation-promoting effect of HMGB1 $(p<0.05)$. These latter effects enabled the reduction of the G1 phase and DNA synthesis.

The present data suggest that YHK has a potential role as a modulator of RAGE and RAGE ligands so to be amenable for potential therapeutic intervention in hepatocellular carcinoma prevention strategies within broader health plans. p65

Keywords: YHK, advanced glycation end products, NF- $\kappa \mathrm{B}$

69

\section{STRATEGIES FOR THE CLEARANCE OF AGE-RELATED LYSOSOMAL RESIDUALS: 7-KETOCHOLESTEROL AND FOAM CELL FORMATION}

Mathieu J, Alvarez P

Rice University 6100 Main St. MS-519 Houston, TX 77005

Lysosomal storage diseases (LSDs) have traditionally been defined as a group of inherited metabolic disorders that are typically the consequence of a deficiency in a single lysosomal enzyme. Often overlooked, however, is lysosomal storage that occurs with increasing age, and that is the result of catabolic insufficiency. These forms of lysosomal storage occur in all individuals to some degree, are chronically rather than acutely cytotoxic, and are increasingly being associated with age-related degenerative diseases. Examples of such substances include lipofuscin, 7-ketocholesterol, and N-retinylidene-N-retinylethanolamine (A2E). As with inborn errors of metabolism, the consequences of age-related lysosomal storage can include volume expansion, enzyme inhibition, reduced protein homeostasis, and altered membrane composition. This may result in the activation of a number of pathological cascades, including apoptosis, oxidative stress, inflammation, and endoplasmic reticulum stress. In an effort to discern the pathological consequences of such lysosomal storage, and provide novel therapeutic avenues for treatment, our group is developing methods to facilitate the selective elimination of unmetabolized substances from the lysosome.

To establish proof-of-concept that the selective removal of intralysosomal substances can help attenuate age-related disease, we first sought to determine the principle component of oxidized LDL responsible for foam cell formation. 7-ketocholesterol $(7 \mathrm{KC})$ is the major form of oxysterol found associated with oxidized LDL, and is highly concentrated in atherosclerotic plaques. Intracellularly, its propensity to concentrate in the lysosome hinders enzymatic transformations that reduce its toxicity and increases sensitivity to lysosomal membrane permeabilization. Using THP-1 monocytes, we have found that 7KC-loaded LDL causes significantly accelerated macrophage differentiation as well as a $50 \%$ increase in both neutral and polar lipid retention when compared to cells treated with normal or moderately oxidized LDL. Importantly, 7KC-differentiated macrophages are primarily of the spindle-shaped, M1 variety, which are considered pro-inflammatory. We assessed the potential to mitigate the effects of $7 \mathrm{KC}$ on foam cell formation by exposing THP-1 macrophages to the cyclic oligosaccharide hydroxypropyl- $\beta$-cyclodextrin $(\mathrm{H} \beta \mathrm{CD})$. We found that $\mathrm{H} \beta \mathrm{CD}$ is effective at reducing monocyte to macrophage differentiation. Furthermore, $\mathrm{H} \beta \mathrm{CD}$ is able to reverse $7 \mathrm{KC}$-mediated lysosomal membrane permeabilization, as determined by lysosomal retention assays. Interestingly, while $\mathrm{H} \beta \mathrm{CD}$ also significantly increased the viability of 7KC-exposed monocytes, it reduced the viability of monocytes exposed to high concentrations of LDL or oxLDL. In order to address this apparent discrepancy, we are also currently exploring the overexpression of both 7KC-active enzymes as well as oxysterol-specific sterol transporters to facilitate 7KC clearance from foam cells.

Keywords: 7-ketocholesterol, oxysterol, low density lipoprotein, lysosome, foam cell

70

\section{THE GENE EXPRESSION PATTERNS OF 'NORMAL' AGING SHOW A HIGH SIMILARITY WITH AGE-RELATED PATHOLOGIES}

Mazuz K, Budovsky A, Fraifeld VE

Faculty of Life Sciences, Achva College, The Shraga Segal Department of Microbiology, Immunology and Genetics, Center for Multidisciplinary Research on Aging, Ben-Gurion University of the Negev, Beer Sheva, Israel

We applied the MARQ (Microarray Rank Query) tool for identifying similar gene expression signatures from human DNA microarray data on age-related changes in various organs. This web-based tool allows comparing a query set of genes against GEO datasets, in order to identify conditions that induce similar or opposite gene expression patterns. Our analysis revealed that the gene expression patterns of 'normal' aging show a high similarity with various pathological conditions including the major age-related diseases (ARDs). This supports our notion that ARDs are not only direct consequences of aging but actually 
represent its diverse manifestations, thus being an essential part of the 'normal' aging process.

This work was funded by the EC FP7 Health Research Grant number HEALTH-F4-2008-202047 (RESOLVE).

Keywords: Gene expression signatures, Aging, Age-related diseases, MARQ software, DNA microarrays

71

\section{HOW DOES THE BODY KNOW HOW OLD IT IS?}

\section{Mitteldorf J}

MIT

The mainstream SENS program is premised on the assumption that the body becomes damaged with age, and it is up to us to fix it because the body cannot repair itself. But there has always been a subculture within the SENS community, those of us who believe that the body retains a latent capacity for repair. There exists the possibility that the major hallmarks of senescence can be addressed using signal molecules to instruct the body to behave as though it were a younger age, that appropriate repairs would then be undertaken as they were when the body was young, obviating the need to engineer artificial fixes for each separate form of damage. This possibility deserves investigation, first because there is evidence to support it, and second because, if it pans out, it will offer a shortcut to longevity, achieving SENS goals with less complication and expense. If we take this idea seriously, it implies that the body knows how old it is, and adjusts its biochemistry accordingly - in other words, the metabolism is modulated by an internal clock. Our task is to understand that clock well enough to intervene and reset it to an earlier age. One clock that has been proposed is based on telomere length. In fact, we know that telomere length is the primary aging clock for some protozoans. Over the last decade, we have learned that mortality risk correlates negatively with telomere length (adjusted for age) in man and most other mammals and birds. Evidence that this association is causal is supplied by recent experiments in which telomerase is demonstrated to rejuvenate telomerase knock-out mice. Another candidate aging clock is implemented through gene expression. Experiments with heterochronic parabiosis - linking the circulatory systems of old and young mice - suggest that proteins circulating in the blood may have the power to rejuvenate as well. The documented differences in gene expression between old and young people may be a driver of senescence and not merely a systemic response. Gene expression is controlled locally within a cell by methylation of cytosine, by acetylation of histones, among other modifiers; central control of gene expression from outside the cell is managed through circulating transcription factors. Direct manipulation of a chromosome's methylation state is not currently possible, but is theoretically feasible. It is an intriguing possibility that the transcription state constitutes a biological clock which can be reset, instructing an old body to behave like a young body.

\section{2}

\section{AGE DYNAMICS OF TRANSCRIPTOMES OF LONG-LIVED DROSOPHILA STRAINS WITH GADD45 OVEREXPRESSION IN NERVOUS SYSTEM}

Moskalev AA, Plyusnina EN, Kudryavtseva AV

Institute of Biology, Komi SC, UB RAS, Syktyvkar 167982, Russia
There is a relationship between various cellular stress factors and aging. Gadd45 proteins stand at the crossroad of the cell fates by controlling the balance between DNA repair, eliminating (apoptosis) or preventing the expansion of potentially dangerous cells (cell cycle arrest, cellular senescence), and maintaining the stem cell pool. However, the biogerontological aspects have not thus far received sufficient attention. Our data revealed an increase of Drosophila melanogaster lifespan due to $D$ GADD45 overexpression in the nervous system as well as the role of D-GADD45 in oxidative, thermal and genotoxic stress resistance. We conducted RNASeq of transcriptomes of flies with conditional overexpression of D-GADD45 and observed differential expression of some evolutionary conservative longevity genes. We also analyzed the pathways and modes of action by which Gadd 45 members are involved in aging, longevity and age-related diseases in mammals. Although further wide-scale research is warranted, it is becoming increasingly clear that Gadd45s are highly relevant to aging, age-related diseases and to the control of life span, suggesting them as potential therapeutic targets in age-related diseases and pro-longevity interventions.

This work was supported by Presidium of the RAS grant 12C-4-1007.

Keywords: transcriptomics, Drosophila, GADD45

73

\section{TOWARDS PRIORITIES FOR AGING RESEARCH}

Needham SL

Leading Technology Group

The global population is aging and age remains the primary risk factor for all major causes of death (COD). Progress in aging research will be essential to reduce disease burden. Funding research into senescent diseases with the highest burdens will bring the greatest benefit to society, yet no specific means to evaluate aging research priorities currently exists. As investors in the life sciences field we reviewed the mortality modelling literature and identified tools to analyse the 2003 Australian mortality data. Two approaches for ranking COD are presented: 1) after adjusting for risk factor-associated deaths and 2) after excluding diseases that decelerate in old age. Such models will improve efforts to target funding to research fields with the most potential to benefit human health.

Keywords: Mortality Trends, Cause of Death, Aging Physiology, Risk Factors

\section{4}

\section{RESCUE OF MTDNA MUTATIONS BY ALLOTOPIC EXPRESSION OF ENGINEERED MITOCHONDRIAL GENES}

O'Connor M, Boominathan A, Vengalam J, Crampton A, Vanhoozer S SENS Research Foundation

Mutations in mitochondrial genes occur as we age, and the human body does not have a good system to repair them. At SENS Research Foundation we are in the early stages of creating an exciting and innovative system to repair mitochondrial mutations. In this project, engineered mitochondrial genes are being used to restore function to cells that have defective mitochondrial genes. 
Recent research suggests that the use of specific targeting sequences attached to engineered mitochondrial genes expressed in the nucleus allows for functional rescue of mutations in mitochondrial DNA. These targeting sequences localize corrected mRNA to the surface of the mitochondria and improve the efficiency of protein import into the mitochondria. Several challenges remain that inhibit the use of allotopic expression as a viable strategy, however, including efficient and stable longterm protein expression necessary for permanent rescue of deleterious mutations.

Our strategy is as follows: (1) recode the mitochondrial genes for nuclear codon usage, (2) target the mRNA to the surface of the mitochondria using $5^{\prime}$ and $3^{\prime}$ end targeting signals, (3) achieve efficient import using a canonical N-terminal mitochondrial targeting sequence (MTS), and (4) demonstrate functional rescue of mitochondrial mutations.

We have analyzed localization of the targeted RNA sequences to the mitochondria by qRT-PCR from isolated mitochondria. To evaluate the ability of these engineered mitochondrial genes to rescue mtDNA mutations, these constructs are being stably expressed in WT and mutant patient-derived cell lines. The successful rescue of mtDNA mutations by targeting exogenous mitochondrial gene products into the mitochondria has tremendous potential for the treatment of the maladies caused by mitochondrial mutations.

75

\section{INNATE AND VACCINE-GENERATED ANTIBODIES AGAINST TRANSTHYRETIN AMYLOID}

O'Nuallain B, Nishiyama Y, Phay M, Planque SA, Hara M, Watanabe K, Blinder V, Paul S

Brigham and Women's Hospital and Harvard Medical School

Aging is associated with the aberrant deposition of sticky extracellular aggregates of amyloid polypeptides in tissues and/or organs. The formation of amyloid is associated with a host of human diseases, including heart disease, Alzheimer's disease, and diabetes. Unfortunately, amyloid diseases are currently incurable, and there is an urgent need for non-invasive diagnostics for timely patient treatment with current symptomatic or future disease-modifying therapies. To address this, we are investigating the therapeutic and diagnostic potential of innate and vaccine-generated antibodies that target toxic amyloid assemblies of a blood transport protein, transthyretin (TTR), the cause of senile systemic amyloidosis (SSA) - a disease that affects $\sim 20 \%$ of humans over the age of 80 years. We will report progress on identifying antibodies that specifically catalyze the breakdown of toxic TTR assemblies. Our experimental approach is based on the hypothesis of covalent catalysis as a primordial immune mechanism that can be applied to identify an efficacious therapeutic agent for treating SSA. We have also vaccinated mice with non-native TTR and generated eight novel murine TTR-reactive monoclonal IgG antibodies that have diagnostic potential for SSA. These IgGs specifically target binding sites on soluble and insoluble non-native TTR assemblies, and can recognize patient-derived TTR amyloid but not native physiologically relevant TTR contained in normal human plasma. Taken together, these findings support further investigations on the therapeutic and diagnostic potential of the innate and vaccine-generated anti-TTR antibodies for SSA.

Keywords: amyloid, antibody, transthyretin, catalyze
76

\section{SOLUBLE TUMOR NECROSIS FACTORS (STNFR1 AND STNFR2) ARE INCREASED IN AN AGE-DEPENDENT MANNER AND IN MULTIPLE ORGAN MALIGNANCIES}

Obrenovich ME, Emancipator SN, Frackelton JP, Lonsdale D Institute of Pathology, Case Western Reserve University

The NCI estimates that 1:2 men and 1:3 women alive today will be diagnosed with a form of cancer in their lifetimes. With the rising worldwide incidence of cancer, it is imperative that better and earlier diagnostic and screening tests. Cancer remains a major contributor especially to morbidity and mortality among elderly patients, as well as the disease is rising in the general population. Much of the progress in the improving survival of cancer patients has derived from improved detection of cancer at earlier stages of disease. A limitation of current screening strategies is that the tests used are limited to a particular malignancy such as endocervical, colorectal, breast, prostate and skin cancers. We have explored the utility of a family of soluble tumor necrosis factor receptors after validating our in vitro diagnostic assay for select members of the TNF superfamily of receptors. The samples in this study consisted of discarded and de-identified serum from normal age-matched control patients, without frank malignancies or known pathology as compared with individuals with various multi-organ malignancies irrespective of grading and level to assess whether screening serum for common proteins released into the bloodstream by a wide range of malignant cells was a novel and useful approach for detection of malignancy. Based on preliminary data, we have demonstrated that select markers, namely: soluble TNF receptor 1 (sTNFRI), soluble TNF receptor 11 (sTNFRII), and others (not discussed here), circulate in serum at increased levels in an age-dependent fashion as well as in cancer patients. Because these soluble factors are produced, or rather shed, by malignant cells and tumors at higher rates than by non-cancerous cells, they are potential targets of therapeutic intervention and have potential for diagnostic utility. The long-term goal is to develop cost-effective, clinical novel screening tests for a wide range of malignancies, our approach is of significant importance as it address the cost obstacle to current approaches, such as financial limitations in whole-body screening and offer tremendous savings and wide application in screening for many malignancies, especially the so-called "silent killers". At the same time lowering these factors by dietary means, such as reduced glycotoxins are another avenue we intend to explore.

Keywords: cancer, early diagnosis, TNFR, screening

77

\section{A NEW TELOMERE/STEM CELL THEORY OF AGING}

Park E

Private practice in Anti-Aging medicine, Orange County, California

Dr. Ed Park is the Harvard and Columbia-trained medical doctor and the author of "Telomere Timebombs: Defusing the Terror of Aging". In 2007, he became one of the first 20 people to regularly ingest a nutraceutical telomerase activator. In 2008, he became the first $\mathrm{MD}$ to be licensed to prescribe it and has since become the foremost expert on what he calls "Telomerase Activation Medicine".

Dr. Park has created over 90 original videos based on his clinical experience that explore a myriad of disease conditions 
and their relationships to telomere erosion. His videos have been viewed over 120,000 times on YouTube.

Based upon his years of clinical experience, he has developed a simple and intuitive new model of aging based on Telomere erosion and Stem Cell biology. He will present this theory and show why it represents a sort of "Grand Unified Theory" of aging and disease that will shift and unify much of the research and efforts that are currently in vogue.

Keywords: telomeres, aging, telomerase, stem cells, dr ed park

78

\section{REDEFINING AND REVERSING DEATH: WHAT HAS RESUSCITATION SCIENCE TAUGHT US ABOUT DEATH AND WHAT HAPPENS WHEN WE DIE?}

Parnia $S$

Stony Brook University School of Medicine, New York

While many concepts regarding the nature of death and hence the questions that relate to human mortality and immortality have existed for millennia, recent scientific advances have indicated that death is in fact a biological and medical process that can now be studied through the objectivity of science. Death has traditionally been defined when the heart stops beating, respiration ceases, and the brain no longer functions as a result of which a person becomes lifeless and motionless. Although it had been considered that brain cells become permanently damaged within 5-10 minutes of cessation of blood flow after death, recent evidence suggests that neurons may remain viable for longer periods of time. As neuronal cell death occurs through a combination of apoptosis, autophagocytosis and necrosis, it may be possible to manipulate these processes and slow down the rate of cell death in the brain and other vital organs following death from circulatory arrest. Thus, while philosophically it has traditionally been considered implausible to reverse death after it has taken place, medically speaking advances in the field of resuscitation science starting about 50 years ago and in particular over the past 10 years have challenged this notion. Current optimal methods include the use of hypothermia, extra-corporeal membrane oxygenation, and mechanical cardiopulmonary resuscitation, while future research indicates a possible a role for direct inhibitors of apoptosis and novel oxygen delivery methods. An unintended consequence of these discoveries aimed at developing new lifesaving measures is that science is also inadvertently expanding our knowledge of death which has major ramifications for any discussion regarding the question of what happens when we die. Millions have now been resuscitated back tens of minutes and at times hours after a period of cardiac arrest and death. For the first time in history, many people have thus gone beyond the traditional threshold of death and have come back to relive and recount their experiences.

Keywords: resuscitation, brain death, viability, critical care

79

\section{TARGETING THE SENESCENCE-ASSOCIATED SECRETORY PHENOTYPE}

Perrott KM, Campisi J

Buck Institute for Research on Aging

Loss of normal tissue homeostasis with age is accompanied by a low but detectable rise in pro-inflammatory factors in the absence of detectable infection. The source of this "sterile" inflammation is unknown however senescent cells have been identified as a possible origin with the identification of the 'senescence-associated secretory phenotype' (SASP) exhibited by many proliferation-capable cell-types in response to genomic damage. Recent studies have shown that removal senescent cells in a rapidly aging mouse model increases healthspan identifying senescent cells and their secretory phenotype as potentially causal in the loss of tissue homeostasis and degenerative processes. Understanding the SASP and discovering ways of reducing its proinflammatory nature could be beneficial in preserving or restoring function to aging tissues. To examine this potential the Campisi lab has screened 1400 FDA approved compounds to identify those able to lower the SASP. The focus of this work is validating and selecting some of the candidate compounds for this ability and determining their mechanism of action. One candidate selected for further characterization and study is the flavonoid Apigenin. Its effects on the SASP of senescent cells will be discussed revealing some novel insights into the secretory profile of senescent cells and how it may contribute to aging phenotypes.

80

\section{REGENERATION OF NEONATAL LYMPHOID ORGANS IN THE OLD ORGANISM}

Pishel IM

D. F. Chebotarev State Institute of Gerontology of NAMS of Ukraine

Age-related changes of the immune system are one of the key factors of entire organism aging. There are several approaches to prevent these changes, such as thymus rejuvenation, stem cells recovery, etc. For this purpose, we used neonatal lymphoid organ transplantation under kidney capsule. This approach enables to form a young lymphoid organ de novo due to the graft resident stem cells being accompanied by migration of circulating recipient's lymphocytes. It has been found that the intensity of regeneration and lymphocyte composition of neonatal spleen graft did not differ in animals of different ages. However, lymphocyte proliferation and PFC number was significantly decreased in the spleen regenerated in old animal. Additional neonatal thymus graft led to the doubling of the transplanted spleen size in old vs young animals, while the lymphocyte proliferation and PFC number were found to increase in animals of both ages but age-related differences still remaind. The obtained results indicate that the de novo organ formation and its function largely depend on the age of the host. The report discusses the possible mechanisms capable to improve immunological functions in aged animals that depend on a combination of transplanted organs and age-related interaction between them.

Keywords: Immunity, aging, neonatal spleen graft

81

\section{UNDERSTANDING THE PARADOX OF OPPOSITION TO LONG-TERM EXTENSION OF THE HUMAN LIFESPAN: FEAR OF FEATH, CULTURAL WORLDVIEWS, AND THE ILLUSION OF OBJECTIVITY}

Pyszczynski T, Greenberg J

University of Colorado Colorado Springs

A large body of psychological research suggests that the fear of death plays an important role in diverse forms of human 
behavior. This research suggests that cultural beliefs and values, and the self-esteem that people get from living up to those values, serves the important function of managing the potential for anxiety that results from human awareness of the inevitability of death. Given people's general disinclination to die, it is paradoxical that many object to the use of science and technology to radically extend the human lifespan. Although there are certainly rational reasons to be concerned about the potential side effects of long-term life extension, the vociferousness of some of these objections suggest that this issue arouses deepseated emotions that might be fueling this opposition. Longterm life extension is likely to challenge both traditional religious and modern scientific conceptions of life that provide people with existential security. If so, this implies that opposition to prolonging life might be rooted, at least in part, in the fear of death and the psychological structures that people use to protect themselves from this fear. This presentation will: 1) provide a broad overview of theory and research regarding the role of death concerns in human allegiance to cultural worldviews, and 2) discuss the interplay between rational and irrational psychological processes likely to promote opposition to long-term life extension research and technology.

Keywords: Fear of death, Terror management theory, cultural worldviews, illusion of objectivity

82

\section{TREATING CYTOPENIA WITH ESC DERIVED BLOOD CELLS}

Rebo J, Webb T, Cook B, Moody K, Fabiny L, Schloendorn J SENS Research Foundation

A variety of cytotoxic insults destroy blood cell progenitors, and the recovery process can take weeks. Transplant of replacement progenitors can immediately restore these cells, but this procedure is rarely done as the only source is bone marrow donors who can provide only a very limited supply. The manufacture of these cells would therefore have great clinical benefit.

Embryonic stem cells (ESCs) can be grown indefinitely. However, previous attempts to derive blood cell progenitors from ESCs have met with limited success: the progenitors thus far produced lacked bone marrow homing ability when transplanted into irradiated mice, and did not protect mice from myelotoxicity.

We have developed a novel differentiation process to address these problems. We produced hematopoietic-colony-forming cells that express CXCR4, the receptor for SDF-1 and key mediator the bone marrow homing pathway, without using any genetic manipulation. We showed that these cells home to the bone marrow and increased survival in mice with cytotoxic induced cytopenia.

Keywords: embryonic stem cells, Regenerative medicine, Cytopenia, preclincial

\section{3}

\section{PANACEA BROAD-SPECTRUM ANTIVIRAL THERAPEUTICS}

Rider TH

Massachusetts Institute of Technology

Although there is great concern over emerging viruses and viruses on the category A-C priority pathogen lists, there are relatively few prophylactics or therapeutics for these viruses, and most which do exist are highly pathogen-specific or have undesirable side effects or other disadvantages. As part of our PANACEA program, we have developed a radically new and very broadspectrum antiviral therapeutic/prophylactic that has the potential to revolutionize the treatment of viral infections, including those due to emerging, category A-C, and common clinical pathogens. Our Double-stranded RNA (dsRNA) Activated Caspase Oligomerizer (DRACO) approach selectively induces apoptosis in cells containing viral dsRNA, rapidly killing infected cells without harming uninfected cells. We have demonstrated that DRACOs are nontoxic in all 11 cell types tested thus far, and effective against 15 different viruses, including DNA and RNA viruses, enveloped and nonenveloped viruses, viruses that replicate in the nucleus and in the cytoplasm, and viruses that use a variety of receptors. Among the viruses against which DRACOs have proven effective in vitro are dengue hemorrhagic fever virus, multiple arenaviruses, and multiple bunyaviruses. In mice, we have demonstrated that DRACOs rapidly penetrate into all organs tested, persist for over 24 hours after each dose, and are nontoxic. We have shown that DRACOs rescue mice from lethal challenges with H1N1 influenza, Tacaribe arenavirus, Amapari arenavirus, and Guama bunyavirus. We hope to optimize our DRACO designs and demonstrate them against additional viruses and in additional animal models. This work should greatly advance DRACOs toward ultimate utility as safe, broad-spectrum therapeutics/prophylactics for priority and emerging viral pathogens, filling a large gap in existing therapeutics.

84

\section{THE DISRUPTIVE POTENTIAL OF TISSUE ENGINEERING}

Russell A

Carnegie Mellon University Distinguished Professor

Tissue engineering is one of the most exciting tools through which regenerative medicine can be accomplished. The concept of growing neo-organs that can cure diseases versus treat symptoms is compelling. This paper will explain why tissue engineering is a fast moving field that brings ideas to the clinic more quickly than the pharmaceutical industry. As a disruptive technology, tissue engineering has struggled to find strong market adoption and this presentation will also summarize the existing challenges in the field and suggest routes that these barriers can be overcome. In particular our own work on membrane engineering of cells to enable cellular therapies and the use of cytotactic surfaces to influence the trajectory of cell rolling will be discussed.

\section{UTILIZING B CELLS TO RECREATE THE BIOCHEMICAL ENVIRONMENT OF YOUTH}

\author{
Herbig E, Xu M, Scholz M \\ Immusoft Corporation
}

New proteins that affect or play a role in aging are continually being discovered. While some of these molecules may have great potential for reversing aging and extending lifespan, current technologies for delivery of biologic proteins are costly and require frequent re-dosing to sustain therapeutic levels. Thus, the field of regenerative medicine is in need of a mechanism that can elicit long-term production of biologics in vivo in a manner that is both cost-effective and clinically practical. Immusoft is currently commercializing technology that turns a patient's own B cells into drug factories that continually produce and secrete 
therapeutic proteins in vivo. Our technology is currently being used for production of monoclonal antibodies and enzymes in the treatment of HIV and rare genetic diseases, respectively. These ongoing efforts are establishing a firm foundation for our future applications in anti-aging and regenerative medicine. In summary, Immusoft's platform offers a vehicle for delivery of critical anti-aging factors and, ultimately, re-creation of the biochemical environment of youth.

Keywords: cell therapy, gene therapy

86

\section{CHROMATIN AND EPIGENETIC DYNAMICS IN SENESCENCE PHENOTYPES}

\author{
Sedioy JM \\ Department of Molecular Biology, Cell Biology and Biochemistry, \\ Brown University
}

Chromatin is a complex and dynamic structure that needs to be maintained in a functional state throughout the lifetime of an organism. Studies of diverse species, including mammals, have revealed that chromatin undergoes extensive rearrangements during aging. Cellular senescence, an important component of mammalian aging, has recently been associated with decreased heterochromatinization of normally silenced regions of the genome. These changes appear to lead to the expression of retrotransposable elements (RTE), culminating in their transposition. RTEs are common in all kingdoms of life, and comprise close to $50 \%$ of mammalian genomes. They are tightly controlled, as their activity is highly destabilizing and mutagenic to the genomes in which they reside. In my presentation I will develop the hypothesis that the "loosening" of our endogenous genomic parasites, the RTEs, is an important and hitherto unexplored molecular aging process that can potentially occur in most of our tissues. We further envision that the activation and continued presence of retrotransposition contributes to age-associated tissue degeneration and pathology.

Keywords: senescence, chromatin, retrotransposable elements, RTEs

87

\section{INTERMITTENT HYPOXIA, OXIDATIVE STRESS AND INFLAMMATION: THE LONG AND STRESSFUL ROAD TO CARDIOVASCULAR DISEASE}

Shannack $M$

King's College, London

Obstructive sleep apnea (OSA) is a condition of sleep-disordered breathing which is an increasing health concern due to independent associations with cardiovascular diseases. An element of OSA, called intermittent hypoxia (IH) is thought to be responsible for many of the detrimental effects of this disorder. In particular, $\mathrm{IH}$ is believe to induce the generation of reactive oxygen species (ROS) which leads to oxidative stress (OS), and inflammation. A combination of OS and inflammation is implicated in the development of endothelial dysfunction, hypertension and arterial stiffness; all of which are significant risk factors for the development of cardiovascular morbidities. This presentation will discuss the putative mechanisms, discovered in cell, animal and clinical studies, by which $\mathrm{IH}$ induces OS and inflammation, and the consequences of these factors to the detriment of cardiovascular health in OSA patients.
88

\section{BIOINFORMATIC ANALYSIS OF FACTORS INVOLVED IN AGE-RELATED MINERALIZATION OF SOFT TISSUES}

Shindyapina AV, Mkrtchyan G, Gneteeva T, Buiucli S, Zhavoronkov A

Lomonosov Moscow State University Moscow Center for Pediatric

Hematology, Oncology and Immunology

Age-related metastatic mineralization of the soft tissues was historically considered to be a passive and spontaneous process. Recent data demonstrate that calcium salts deposition in soft tissues could be a highly regulated process. Mechanisms leading to this pathology vary in different patients and depend on disease type, stage, patient gender, and age.

Although calcification occurs in any tissue type, vascular calcification has been of particular interest due to association with atherosclerosis, chronic kidney disease (CKD), and osteoporosis. Patients with these age-related disorders explore different mechanisms underlying calcium apatite accumulation. In case of atherosclerotic plaque oxy-lipids triggers release of the pro-inflammatory cytokines and inflammation that activate calcification processes in intimae aorta. Recent in vitro study demonstrates that oxy-lipids can accelerate vascular smooth muscle cells (VSMC) calcification in a dose-dependent manner. In CKD patients renal failure alter the balance between calcium and phosphate levels that usually regulated by FGF23, Klotho, and vitamin D. With accumulation of both phosphate and calcium ions VSMC begin to explore osleoblast-like phenotype. Finally, bone-derived matrix vesicles in osteoporotic patients can degrade extracellular matrix of tissues and accelerate calcium apatite growth.

There are many processes leading to calcification of structural cells and extracellular matrix. Collagen is a major component of extracellular matrix and modifications of collagen fibers accumulate with age. Formation of cross-links between collagen fibers are regulated by action of the lysine hydroxylases and lysyl oxidase, and could occur spontaneously. Oxidation-induced AGEs is a major type of spontaneous cross-links that accelerate with age and may result in tissue stiffness, problems with recycling and potentially accumulation of calcium apatite. The accumulation of AGEs and the rate of mineralization may be interrelated. Applying strategies for clearing the AGEs proposed by de Grey, may be more difficult in the highy-mineralized extracellular matrix.

We performed bioinformatic analysis of the molecular pathways underlying calcification in atherosclerotic and CKD patients, signaling pathways of collagen cross-links formation and bone mineralization and proposed new potential targets for calcification treatment and review drugs for the targets.

Keywords: Metastatic calcification, Collagen cross-linking, Vascular calcifiaction, Systematic review

89

RANGE OF VARIATION IN THE DURATION

OF C. ELEGANS DAUER AND POST-DAUER SURVIVAL: FOOD INTAKE OF PARENTS AND FIRST-STAGE LARVAE AFFECTS ADULTS RECOVERED AFTER 60 DAYS OF DEVELOPMENTAL ARREST

Balasubramaniam M, Ayyadevara S, Shmookler-Reis R University of Arkansas for Medical Sciences, and Central Arkansas

Veterans Healthcare Service, Little Rock AR, USA 72205 
The nematode C. elegans, when maintained under crowded and/ or starved conditions (or upon exposure to a hormonal mixture termed "daumone"), enter an alternative developmental stage called the dauer larva. This is a motile but non-feeding state of developmental arrest, which is considered "non-aging" because worms that resume development, after as much as 2 months in dauer status, survive as adults with the same lifespan as worms that never entered dauer [1]. Recovery diminished between 60 and 90 days of dauer arrest, and adult lifespan was not tested beyond 60 days [1]. Aubrey de Grey postulated that dauer formation, like insect diapause and hibernation in mammals, is an essential strategy for over-wintering and survival of periods of famine [2], and thus may afford a fixed additional period of survival rather than a proportionate increase across species. We asked whether variation in the pre-dauer diet $(0.1-2$ OD bacteria) or the mode of dauer induction could extend the period of dauer survival from which fertile adults emerge. Would varying caloric or lipid intake, or the means of dauer induction (daumone vs. starvation), affect the accumulation of fat stores and thus survival potential? Adult lifespan was maximal in recovered adults (after 1 or 2 months in the dauer state) that had undergone moderate caloric restriction ( 0.6 or 1 OD bacteria) prior to dauer formation. After 1 month in dauer, peak mean adult lifespan was $20 \mathrm{~d}$ after recovery from starvation-induced dauer state, and 21 days after daumone induction of dauer; in each case the optimum prior bacterial density was at 0.6-OD. Survival was significantly worse after 2 months in dauer, with peaks (17.8 and 15.7 days respectively) shifted to 1-OD bacteria, and daumone-induced dauers were then much shorter-lived (and less CR-responsive) than starvation-induced dauers. Survival of oxidative stress was greatest in adults recovered after 1 or 2 months in the dauer state, if they had undergone caloric restriction to $0.3,0.6$ or $1 \mathrm{OD}$ bacteria prior to dauer formation, with lower stress resistance after 0.1 or 2.0 OD. Like lifespan, stress survival was substantially worse after 2 months in the dauer state. Fecundity (total egg production) was minimal for recovered adults that had previously seen 0.6 OD bacteria, showing an inverse relationship to lifespan.

1. Klass M, Hirsh D (1976) Non-ageing developmental variant of Caenorhabditis elegans. Nature 260: 523-525.

2. de Grey AD (2005) The unfortunate influence of the weather on the rate of ageing: why human caloric restriction or its emulation may only extend life expectancy by 2 - 3 years. Gerontology 51: 73-82.

Keywords: C. elegans, dauer, calorie restriction, lifespan, survival

\section{0}

\section{AGED MICE REPEATEDLY INJECTED WITH PLASMA FROM YOUNG MICE: A SURVIVAL STUDY}

\author{
Shytikov DW, Balva OV, Pishel IM, Debonneuil E \\ D.F. Chebotarev State Institute of Gerontology NAMS, Kyiv
}

The deterioration of functions of various organs and systems with age is well documented. Numerous violations in metabolism, in the cardiovascular system and the immune system lead to the development of specific diseases associated with aging that are the main cause of death of people in older age groups.

In a classical work by Carrel et al., serum from old animals was found to inhibit the growth of cell cultures. More recently, Wyss-Corray et al. showed that injection of plasma from older animals can cause age-related changes in the nervous system of young recipients. This and other work suggest that transferring plasma from old animals can induce some type of aging. In several recent works it has been shown on injections of plateletrich plasma may be beneficial in several pathologic conditions. The question remains opened whether transferring plasma from young animals can induce some type of rejuvenation or be beneficial for aging mice.

The aim of the present study was to investigate the presence of a positive effect of chronic injections of young animals' blood plasma on the lifespan and on markers of aging of experimental animals.

A survival study was performed with 11-13 month old female CBA/Ca mice. An experimental group $(n=36)$ was repeatedly injected with heparinized plasma from young CBA/Ca females (2-4 month old). Control group was injected with heparinized saline. Injections were performed over 15 months with approximately one intravenous and one intraperitoneous injections per week and doses ranging from 75 to $150 \mathrm{mcl}$. In addition to survival assessment, necropsies of dead animals were made and blood samples were taken from aged mice at $t=6.5,8,9,14$ months of experiment to analyze $\mathrm{CD} 4 / 8$ ratio and total $\mathrm{T} 4$ level as these parameters are the most affected with age.

No statistical difference in lifespan was observed between the experimental and control groups; the median lifespan of the control group was 27 months which suggests good conditions. The CD4:CD8 ratio was statistically lower in the experimental group starting from 20 months age and this tendency was present for several months later, which in general suggest a weaken immune system, and the effect became weaker with time. In parallel with a low CD4:CD8 ratio, T4 levels were elevated in plasma-treated mice after 8 months of the experiment, without fully reaching statistical significance comparing to control group, and the effect became weaker with time. Necropsies found out more tumor pathologies in mice treated with plasma from young animals and less of other causes.

Conclusions. In the conditions of the experiment no positive effects of injections of plasma from young animals to animals of older age groups were found. And the prevalence of cancer pathology in plasma-treated mice suggests possibility that exposure of youth factors in aged systemic environment may be even harmful for aged host.

Keywords: Plasma injection, Survival rate, $\mathrm{CD} 4 / 8$ ratio, Thyroxin level

91

\section{ALTERED CANCER CELLS: UNCOVERING THE GENETIC BASIS OF ALT (ALTERNATIVE LENGTHENING OF TELOMERES)}

\author{
Halvorsen D, Hunt T, Moody K, Silva H \\ SENS Research Foundation
}

As normal cells divide, the ends of their chromosomes (telomeres) progressively shorten until eventually the cells reach senescence or undergo apoptosis. Cancers, which disproportionally kill more individuals in the 65 years or above age group, often overcome this built-in replication limit by expressing the enzyme telomerase.

However, about $10-15 \%$ cancers do not use telomerase and at least a major subset of these exhibit hallmarks of Alternative Lengthening of Telomeres (ALT) activity, including (1) long and heterogeneous telomere lengths, (2) presence of ALT-associated PML nuclear bodies (APBs), and (3) generation of high-levels of $\mathrm{C}$-rich circular telomeric DNA repeats (C-circles). Although 
there are many telomerase-based anti-cancer therapies in clinical development at the moment, research on ALT has not produced any promising therapies so far. This lag is due in part to a lack of assays that are reliable and amenable to high-content/highthroughput (HTS) screens.

The OncoSENS team has made significant progress toward making some of these key ALT assays compatible with the HTS format, which should not only speed up the development of ALT-based anti-cancer therapies but also broaden the amount of research performed on ALT. Our team has already begun applying the assays above to test the involvement of several genes in the ALT pathway and the progress on that front will also be showcased. Undoubtedly, successfully shutting down both ALT- and telomerase-based pathways of telomere maintenance in cancers will move the field forward towards realizing the goal of a complete eradication of one of the main age-related fatal diseases burdening society.

Keywords: telomeres, telomerase, ALT, cancer, genetic mechanisms

92

\section{NICOLAI SIETNICKI - A NICOLAI FEDOROV FOLLOWER AS TRANSHUMANIST PRECURSOR OF A DOGMATIC PHILOSOPHY OF THE EXTENSION OF LIFE CONCEPT}

Sitnicki I

not-provided

Nicolai Sietnicki (1888-1937), also spelled Setnitsky, scion of Polish noble family bearing Nałęcz coat-of-arms. Born in Olgopol, Volhynia, Ukraine. After graduating from a classical gymnasium, he completed studies at Saint Petersburg Imperial University. Graduate of both law and sinology faculties, he also studied physics, mathematics and statistics. After military service in WW I, he worked in Odessa. The collapse of the Russian Empire and Bolshevik Revolution forced him to emigrate into Harbin, Manchuria, where he worked for Transsiberian Chinese Railway and later took a post as professor of law at Russian University in Harbin, where he briefly served as chancellor. He was close follower of Nicolai Fedorov concept of the extension of life and overcoming mortality from a dogmatic standpoint. In his book written together with Alexander Gorski - "Adoration of Death" (Harbin, 1926), he defended the view that adoration of death within christianity is a misconcept which is contradictory to christian learnings e. g. dogmas of death overcoming and positive eschatology. According to Sietnicki extension of life is possible from dogmatic and religious point of view and his idea of "universal cemetery" (necropolis universalis) might be seen as synonymous of "genetic bank" of mankind. Other major works by Sietnicki were "On final ideal" (Harbin, 1932), "Statistics, lterature and poetry" (Odessa, 1922) and others. He was the editor of complete works of Nicolai Fedorov - "Philosophy of the Common Task" (Riga, 1934). In 1935 Sietnicki had been promised a cathedral of philosophy at Moscow State University, but instead of this was executed in 1937.

93

\section{TARGETED MITOCHONDRIAL THERAPEUTICS IN AGING}

Smigrodzki R, Portell RF, Onyango I, Dennis J, Khan SM Gencia Corporation, Charlottesville VA
Mitochondrial dysfunction in aging consists of relative suppression of oxidative phosphorylation and frequently an increase in glycolysis. This metabolic imbalance is triggered by progressive biochemical processes, including accumulation of mitochondrial mutations, and changes in the expression and function of nuclearencoded mitochondrial proteins. Our group developed methods for mitigation of mitochondrial suppression through mitochondriatargeted therapeutics. We observed that stimulation of mitochondrial activity both in vitro and in vivo significantly improves cellular function, suppresses neoplastic growth and inflammation, improves aged animal cognition and resolves in vivo metabolic derangements. One of our therapeutics, an engineered mitochondrial transcription factor, prolonged survival in wild-type aged animals. We expect that mitochondrial stimulation will be an important part of future aging therapies.

94

\section{LIFE-LONG PERSISTENT VIRAL INFECTION ALTERS THE NAÏVE T-CELL POOL, IMPAIRING CD8 T-CELL IMMUNITY IN LATE LIFE}

\author{
Smithey MJ, Li G, Venturi V, Davenport MP, Nikolich-Zugich J \\ University of Arizona, United States
}

Persistent CMV infection has been associated with immune senescence. To address the causal impact of life-long persistent viral infection on immune homeostasis and defense, we infected young mice systemically with murine CMV (MCMV) and studied their T-cell homeostasis and function over the lifespan. Upon ListeriaOVA infection, 23 month-old animals that had experienced lifelong MCMV infection showed impaired bacterial control and CD8 T-cell function compared to age-matched, uninfected controls. Lifelong MCMV infection was associated with reduced naïve CD8 T-cell precursors, above the loss attributable to aging. Moreover, the OVA-specific CD8 T-cell repertoire recruited after Listeria challenge was entirely non-overlapping between age-matched control and life-long MCMV + mice. Our study for the first time causally links life-long MCMV infection to disturbances in the Tcell repertoire, which corresponds to impaired immunity to a new infection in late life.

95

\section{CHEMICAL AND BIOLOGICAL APPROACHES TO UNDERSTANDING ADVANCED GLYCATION END-PRODUCTS (AGES)}

Spiegel DA

Yale University Departments of Chemistry and Pharmacology

Advanced glycation end-products (AGEs) are a complex class of non-enzymatic post-translational modifications of proteins that have been associated with numerous age-related health deficits. Because they form as complex mixtures in the body, however, they have been difficult to isolate and study in chemically homogeneous form. This presentation will focus on our laboratory's efforts to synthesize and study chemically pure AGEs including the methylglyoxal-derived hydroimidazolones (MG-Hs) and glucosepane. These studies have led to several novel discoveries, which have significant potential to facilitate the development of therapeutics to prevent age-related health problems, and to enable our fundamental understanding these processes.

Keywords: Advanced Glycation End-Products (AGEs), Glycation, Post-translational modification 
96

\section{ON THE HISTORY OF LIFE-EXTENSION RESEARCH: DOES THE WHOLE HAVE PARTS?}

Stambler I

Department of Science, Technology and Society, Bar Ilan University, Ramat Gan, Israel

This presentation will explore the history of life-extension research in the $20^{\text {th }}$ century. I will argue that the search for life-extension has often constituted a formidable, though hardly ever acknowledged, source for biomedical research and discovery, in such diverse fields as endocrinology (Steinach, 1910s-1920s); probiotic diets (Metchnikoff, 1900s); blood transfusion (Bogdanov, 1920s), systemic immunotherapy (Bogomolets, 1930s-1940s) and cell therapy, including human embryonic cell therapy (Niehans, 1930s-1950s). This presentation will briefly recapitulate some of the biomedical interventions (actual or potential) which were investigated as possible means for life-extension over the century. A taxonomy will be suggested between reductionist/therapeutic and holistic/hygienic approaches to potential life-extending interventions. Both approaches sought to achieve biological equilibrium and constancy of internal environment, yet emphasized diverging means and diverging perceptions of what constitutes equilibrium and constancy. The reductionist approach saw the human body as a machine in need of repair and internal adjustment and equilibration, seeking to achieve material homeostasis by eliminating damaging agents and introducing biological replacements, in other words, working by subtraction and addition toward balance. The holistic approach, in contrast, focused on the equilibration of the organism as a unit within the environment, strongly emphasizing the direct sustaining and revitalizing power of the mind and hygienic regulation of behavior. In the holistic approach, internal equilibrium was sought not so much through calibrating intrusions, but through resistance to intrusions. The apparent relative weight of each approach in public discourse will be shown to change with time, in several western countries, reflecting the initial hopes, disappointments and reactions to those disappointments in a variety of scientific programs.

Keywords: Life extension, history of medicine, reductionism, holism, homeostasis

\section{TRANSPLANTATION OF IN VITRO DERIVED MICROGLIA}

Stolzing A

Fraunhofer Institute for Cell Therapy and Immunology, Germany

Alzheimer's disease (AD) is an age-related neurodegenerative disease associated with the formation of amyloid plaques, tau aggregation and oxidative/inflammatory damage. Microglia play an important role in the early phase of the disease and are known to be involved in AD progression. Senescent microglia accumulate in AD causing inflammation, neuronal damage and increasing the $A B$ load. Microglia could be an attractive target for replacement/rejuvenation cell therapies.

Bone marrow derived from wild type and GFP transgenic black / 6 mice ( aged 3 month) was differentiated into microglia in vitro and injected into the tail vein of APP/PS1 Alzheimer mice ( $\sim 12$ months).

After 4 weeks recipient animals and controls were sacrificed, brain tissue was isolated and examined by immunohistochem- istry, PCR and western blott for amyloid pathology, distribution of transplanted cells and inflammation markers.

In addition, male-to-female transplantation was applied to track the transplanted cells using y-chromosome PCR.

Microglia and astrocyte morphology and activation status was analysed using histology and stereology for quantification of changes. Stereology was also used to quantify the reduction of amyloid plaque numbers and size.

Expression of various inflammatory genes was analysed via PCR and western blot. Changes in the short term memory of the recipient animals was analysed using an object recognition test.

I present initial findings and conclusions for the prospect of microglia cell therapy in AD.

Keywords: Alzheimer's disease, microglia, cell therapy, differentiation, stem cells

98

\section{SYSTEM MODELING OF GENETIC FACTORS THAT INDUCE PLURIPOTENCY}

Sukhera UA, Nawaz MS

COMSATS Institute of Information Technology

The advent of Induced Pluripotent Stem Cells has significantly increased the pace of the research in regenerative medicine and stem cell domains. Even though much work has been accomplished but still challenges remain to find the best genes used to induce pluripotency. The objective of this research is to find out the best "cocktail" of reprogrammable factors that induce pluripotency and link them through expression pathways and in silico approaches to associate all in a simplified System model. We designed a simplified methodology based on automated literature data mining, conserved synteny, KEGG pathway analysis, interaction mapping, gene orthology and microarray expression analysis. The results yielded several interesting facts including a new set of pluripotency factors that can hopefully serve as viable replacements for the initial sets and moreover provided us with an information grid to concisely read and judge the different pluripotency factors according to efficacy.

Keywords: Induced Pluripotent Stem Cells, System Model, Pluripotency, Yamanaka Factors, Reprogrammable Factors

99

\section{PREDICTION OF NEW LIFESPAN REGULATORS IN C. ELEGANS}

Tacutu R, Budovsky A, de Magalhães JP, Fraifeld VE Integrative Genomics of Ageing Group, Institute of Integrative Biology, University of Liverpool

Many studies in model organisms have successfully focused on identifying the genetic factors that determine lifespan and so far hundreds of longevity-associated genes (LAGs) have been discovered [1]. Yet, the search is still on. Biological processes often revolve around protein complexes and are fine-tuned by regulatory factors. As such, the first-order protein-protein interactors of known LAGs are likely to participate in the regulation of lifespan and are more likely to have longevity phenotypes than randomly chosen genes. Moreover, many of these longevity interactors are essential for development and growth. According to the Antagonistic Pleiotropy Theory of Aging, some of the genes that have a beneficial effect early in life could exert detrimental effects at postdevelopmental stages. Recently, this hypothesis received a strong 
experimental validation from a post-developmental RNAi screen in C. elegans [2]. Our results suggest that longevity networks are functionally relevant, and combined with the selection of genes fitting antagonistic pleiotropy, possess a high predictive power for identifying new lifespan regulators.

Participation to the conference was supported by the Marie Curie FP7-PEOPLE-2011-IEF Fellowship within the 7th European Community Framework Programme.

[1] Tacutu R, Craig T, Budovsky A, Wuttke D, Lehmann G, Taranukha D, Costa J, Fraifeld VE, de Magalhães JP. Human Ageing Genomic Resources: integrated databases and tools for the biology and genetics of ageing. Nucleic Acids Res. 2013 Jan;41(Database issue):D1027-33.

[2] Tacutu R, Shore DE, Budovsky A, de Magalhães JP, Ruvkun G, Fraifeld VE, Curran SP. Prediction of C. elegans longevity genes by human and worm longevity networks. PLoS One. 2012;7(10):e48282.

Keywords: longevity, networks, prediction

100

\section{THE LINKS BETWEEN POLAR MRNA TRANSPORT AND LIFESPAN IN BUDDING YEAST SACCHAROMYCES CEREVISIAE}

Taranukha D, Budovsky A, Gobshtis N, Braiman A, Porat Z, Aronov $S$, Fraifeld VE

The Shraga Segal Department of Microbiology, Immunology and Genetics, Center for Multidisciplinary Research on Aging, BenGurion University of the Negev, Beer Sheva, Israel

The budding yeast Saccharomyces cerevisiae is a unique model for studying the links between aging and rejuvenation. These two processes are coupled through specific regulatory mechanisms of the polar protein transport between the mother (aging) and daughter (rejuvenating) cell. Here, we examined a still unexplored possibility for co-regulation of polar mRNA transport and lifespan. To monitor the amount and distribution of mRNAcontaining granules in mother and daughter cells, we used a fluorescent mRNA-labeling system, with MFA2 as a reporter gene. The results obtained showed that deletion of the selected longevity regulators in budding yeast had a significant impact on the polar mRNA transport. This included changes in the amount of mRNA-containing granules in cytoplasm, their aggregation and distribution between the mother and daughter cells. A significant negative correlation was found between strain-specific longevity, amount of granules and total fluorescent intensity both in mother and daughter cells. As indicated by the coefficient of determination, approximately $50-75 \%$ of variation in lifespan of the yeast set examined could be attributed to the differences in polar mRNA transport.

This work was funded by the EC FP7 Health Research Grant number HEALTH-F4-2008-202047 (RESOLVE).

Keywords: Lifespan, mRNA polar transport, Budding yeast

101

\section{WHAT REASSURANCES DO THE COMMUNITY NEED REGARDING LIFE EXTENSION? EVIDENCE FROM STUDIES OF COMMUNITY ATTITUDES AND AN ANALYSIS OF FILM PORTRAYALS}

Underwood M, Hall W, and Bartlett $H$

School of Social Science The University of Queensland Australia
It is increasingly recognised that community attitudes impact on the research trajectory, entry and reception of new biotechnologies. Yet biogerontologists have generally been dismissive of public concerns about life extension. There is some evidence that biogerontological research agendas have not been communicated effectively, with studies finding that most community members have little or no knowledge of life extension research. Perhaps because of this lack of knowledge, community members' attitudes and concerns reflect issues raised in popular portrayals of life extension (e.g. in movies). In order to investigate how popular portrayals of life extension may influence community attitudes we conducted an analysis of 19 films depicting human life extension across different genres. We focussed on how the pursuit of life extension was depicted, how life extension was achieved, levels of interest in life extension among characters in the films and experiences of extended life both at an individual and societal level. This paper compares the results of this analysis with the literature on community attitudes to life extension, and makes recommendations about the issues in which the public may require reassurance if they are to support and accept life extension technologies.

102

\section{STEM AND PROGENITOR CELL SENESCENCE IN AGING}

van Deursen, JM

Mayo Clinic

Senescent cells accumulate with aging and have been causally implicated in aging, but which cell types are subject to cellular senescence and contribute organ/tissue remains poorly understood. Mutant mice with low amounts of the mitotic checkpoint protein BubR1 accumulate large amounts of p16Ink4a positivesenescent cells in eye, skeletal muscle, and fat, at a young age and develop age-related pathologies in these tissues. Clearance of senescent cells from these animals, either by genetic inactivation of p16Ink4a or drug-induced apoptosis considerably delays premature aging. To understand the mechanism of senescence mediated tissue dysfunction in this progeroid model we FACS sorted various cell types from skeletal muscle and fat of BubR1 progeroid mice and analyzed them for the presence of senescence markers. Our preliminary data indicate that committed progenitors rather than stem cells or terminally differentiated cell types express p16Ink4a and other senescence markers. The potential implication of these unexpected findings on current models of regenerative decline with aging will be discussed.

Keywords: senescence, p16Ink4a, senescent cells, progenitor cells

103

\section{IMPROVED TECHNOLOGY FOR ORGAN CRYOPRESERVATION BY VITRIFICATION}

van Sickle $S$, Jones $T$

Arigos Inc.

Cryopreservation by vitrification is the current state of the art in experimental organ preservation for transplant. Unlike methods in currently clinical use which only preserve large vascular organs in transplantable condition for hours, vitrification and storage below the glass transition temperature $(\sim-130 \mathrm{C})$ would enable safe storage for years or decades. Success with organ cryopreservation by vitrification would permit true banking of 
organs, increasing the number available, improving immune matching, and reducing wait list times. Two major limitations to this method are thermomechanical fracturing and the inherent biochemical toxicity of vitrification solutions. Arigos Biomedical is developing new technology that can both eliminate fracturing and reduce the effective toxicity of vitrifiable cryoprotectants. Preliminary experiments have demonstrated elimination of fractures in vitrified swine kidneys, as well as dramatically improved cooling rates from 0 to $-100 \mathrm{C}$, which reduce exposure time to cryoprotectant solutions.

Keywords: cryopreservation, organ banking, fracturing, cryoprotectant toxicity, organ transplantation

\section{4}

\section{NUTRACEUTICAL PROPERTIES OF EXTRAVIRGIN OLIVE OIL: A NATURAL REMEDY FOR AGE-RELATED DISEASE?}

Virruso C, Accardi G, Colonna-Romano G, Candore G, Vasto S, Caruso $C$

Laboratory of Immunopathology, Department of Pathobiology and Medical and Forensic Biotechnologies, University of Palermo

The health benefits of the Mediterranean Diet can be largely ascribed to the nutraceutical properties of extra-virgin olive oil (EVOO). Monounsaturated fatty acids (MUFA) such as omega-3 and various phenolic compounds such as oleocanthal, oleuropein, hydroxytyrosol and tyrosol are the main nutraceutical substances of EVOO. These substances have been suggested to have the ability to modulate ageing- associated processes. In experimental models, it was shown that EVOO with high concentration of poliphenols has antiinflammatory and antioxidant properties. Indeed, it was observed that hydroxytyrosol, as well as oleocanthal, inhibit the cyclooxygenases (COX-1 and 2), responsible for prostaglandin production; oleuropein is a radical scavenger that blocks the low-density lipoproteins (LDLs) oxidation.

Due to the relevance of the olive oil in the economy of Sicily, our group has been funded to assess the nutraceutical properties of different kinds of olive oil. Indeed, the aim of the study is to evaluate effects of EVOOs, with low (L-EVOO) and high (HEVOO) poliphenols content, on the immuno- inflammatory and oxidative stress responses in young and old people.

The parameters to be examined will be the inflammatory mediators C-reactive protein (PCR), interleukin 1 (IL-1), interleukin-6 (IL-6) and tumor necrosis factor-alpha (TNF-alpha); while, to evaluate the effect on oxidative stress response LDL oxidation, advanced glycation end-products (AGE) and 8-hydroxy-2-deoxyguanosine will be measured. All the analyses will be made at the baseline and after two months.

The results of the study will be useful to produce olive oil enriched in nutraceutical properties, likely helpful in the prevention of age-related disesases.

Keywords: Olive Oil, Inflammation, Oxidative Stress, agerelated diseases

105

\section{DIABETES TYPE 2 AND CANCER: AN INTEGRATIVE APPROACH TO PREVENTION, TREATMENT AND REHABILITATION}

Voronina T, Grechko N

Tamara Voronina (MD Ukraine), self employed, resident in London The London Rejuvenation Clinic; Grechko N (MD Ukraine), Healthcare International Pharmaceutical Company, London, UK
Diabetes type 2 (D2) and cancer have some similar characteristics in pathogenesis. D2 and cancer are misfolded protein (MPr) diseases. The main preventive actions for both should be supporting the effectiveness of mitochondria (not supplying excess of glucose and oxygen) and reducing the cellular effects of MPr (toxic, non-functional, related to infections, p53 mutation protein). Our approach combines Intermittent Caloric Restriction (ICR) and Intermittent Hypoxia Therapy (IHT), which can synchronise body functions and support the effectiveness of mitochondria.

The 42 patients involved in the trial had a different period of fasting over 24 hours: the first group of 22 patients fasted 12 hours overnight, the second, of 16, 16 hours and the third, of 4, for 18 hours. The treatment of the first and second groups continued for 10-16 days. All patients were given ICR, IHT, acupuncture, oral hypoglycemic agents, if required, and responded well to the treatment. The most remarkable result was achieved with the patients in the third group. 60 years old Vahan E. has been without D2 for the last 12 years; he had D2 for 6 years previously. David M., 73 years old with a lung abscess received 25 sessions during the 27 -day treatment. The abscess was cured during that period. Joanne T., 60 years old, insulin reduction from 180 units to 36 within the 5 day treatment. She had been taking about 35 units until the last observation in 2005. Ron G., 62 , D2 and psoriasis, received the treatment over 3 months in 2005; he lives without diabetes and psoriasis at present. Conclusions: The cellular effects of MPr provide a common framework that may contribute to guide preventive, treatment and rehabilitation strategies of D2 and cancer as two ageing diseases.

Keywords: intermittent fasting, calorie restriction, intermittent hypoxia therapy, diabetes, acupuncture

106

\section{WEB INTELLIGENCE TO SOLVE AGING}

Wuttke D, Kulaga A

University of Liverpool, UK / University of Rostock, Germany

Aging is a complex phenomenon that requires an engineering approach in order to be solved. We initialized the construction of a digital decipher machine, Denigma [http:/denigma.de], to reverse-engineer the aging process, make aging research more collaborative as well as efficient and facilitate the discovery of powerful therapeutics.

Denigma is an open-source platform that uses advanced information technologies for the creation of a web intelligence. It provides an ecosystem of domain-specific tools and applications for the establishment of a comprehensive knowledge base rendered amenable to global computing. For this we utilize both the power of machine learning algorithms and techniques as well as crowdsourcing approach.

Denigma unifies data on aging and structures it in such a way that it is accessible to reasoning and inference by machine algorithms which are complemented by the "power of the crowd". Our crowdsourcing infrastructure is made to attract longevity activists to help scientists, complement and improve machine learning where humans do what machine cannot do well.

We will start our report with explaining the applicability of various machine learning techniques and ways of doing crowdsourcing in aging research and possible valuable outcomes. Then we will show the results that we and our collaborators achieved by using and developing Denigma platform. Finally we will give a demonstration of the workflows of aging researchers, activists and organizations with the system. 
The combination of machine and human intelligence is crucial and extremely important for reverse-engineering the aging process and developing effective therapies to reverse aging together.

Keywords: reverse-engeneering, information technology, machine-learning, crowdsourcing, web intelligence

107

\section{SENESCENT CELLS-BAD NEIGHBORS FOR CANCER CELLS?}

Yanai H, Tacutu R, Budovsky A, Caliò A, Chilosi M, Fraifeld VE The Shraga Segal Department of Microbiology, Immunology and Genetics, Center for Multidisciplinary Research on Aging, Ben-Gurion University of the Negev, Beer Sheva, Israel

Since Hayflick's discovery of the phenomenon of cellular senescence (CS), the contribution or even relevance of this phenomenon to organismal aging has been a subject for continuous debates. Although the question still remains open, an increasing amount of evidence indicates that CS could have a role in aging and age-related diseases (ARDs), rather than being just a laboratory phenomenon. In fact, the current situation in the field could be defined as an attempt to understand to what extent and how is CS involved in aging and ARDs. CS has primarily been suggested as an anti-cancer mechanism. Yet, senescent cells could also have a dual (antagonistic) role-" good citizens, bad neighbors" (Campisi. 2005). The links between CS and cancer are further strengthened by our data pointing to a possible co-evolution of CS and cancer, and that cancer genes seem to stand at the crossroad between CS and other ARDs. We have recently found that the presence of senescent-like cells in tumor tissueis of prognostic value in case of Hodgkin's Lymphoma. These cells expressed CS markers such as SA-beta-galactosidase, the CDKinhibitors $\mathrm{p} 21^{\mathrm{CIP} 1 / \mathrm{WAF} 1}$ and $\mathrm{p} 16^{\mathrm{INK} 4 \mathrm{a}}$, exhibit the features of CS morphotype, and correlate with outcome of the disease. It appears that modulating CS may offer an additional therapeutic tool for combating cancer and other ARDs.

This work was funded by the EC FP7 Health Research Grant number HEALTH-F4-2008-202047 (RESOLVE).

Keywords: Cellular senescence, Cancer, Age-related diseases

\section{8}

\section{MITOCHONDRIAL QUALITY CONTROL: LINKS TO PARKINSON'S DISEASE AND AGING MODELS}

Youle RJ

NINDS, NIH

The products of two genes mutated in autosomal recessive forms of Parkinson's disease, Pink1 and Parkin, have been identified in Drosophila to work in the same pathway to maintain healthy flight muscles and dopaminergic neurons. PINK1 is a kinase located on mitochondria whereas Parkin is an E3 ubiquitin ligase normally located in the cytosol. Upon mitochondrial damage Pink1 recruits cytosolic Parkin to mitochondria to mediate mitophagy revealing a cell biology pathway in mammalian cells where Pink1 works upstream of Parkin. Although PINK1 has a predicted mitochondrial import sequence, its cellular and submitochondrial localization remains unclear in part because it is rapidly degraded. Pink1 appears to be constitutively targeted to mitochondria and cleaved by PARL. Upon membrane damage it becomes stabilized on the outer mito- chondrial membrane to recruit Parkin. Thus Pink1 serves to flag damaged mitochondria for removal. Whole genome RNAi screens reveal new gene products involved in regulating PINK1 targeting to mitochondria and Parkin translocation to mitochondria. How defects in mitochondrial quality control pathways exacerbate mitochondrial stressors in vivo in mice will be presented.

109

\section{NEW ECONOMIC ARGUMENTS FOR ACCELERATING AGING RESEARCH}

Maria Litovchenko, Alex Zhavoronkov

The Biogerontology Research Foundation, UK and Moscow Institute of Physics and Technology, Russia

While the doubling of the life expectancies in the developed countries in the 20th century can be attributed mostly to decreases in child mortality, trillions of dollars spent on the biomedical research by the governments, foundations and corporations over the past sixty years are yielding longevity dividends in the older population. When increases in life span will occur after the retirement age and extend the "last mile", unless the retirement age is proactively adjusted, the net economic effect of these increases is negative. We introduced several new parameters that can be applied to the established models of the economic growth: the biomedical progress rate, rate of clinical adoption and the rate of change in retirement age. The biomedical progress rate is comprised of the rejuvenation rate, extending the productive lifespan and the non-rejuvenating rate, extending the lifespan after the age at which the net contribution to the economy becomes negative. By extending the Solow model we provided an example of the relations between these new parameters in the context of the demographics, employment and labor, households and the firm. The new model provides new economic arguments for refocusing the research activities towards projects that increase healthy productive lifespan.

Keywords: aging economics, ageing economics, economics of longevity, life span economics

110

\section{P53-DEPENDENT RELEASE OF ALARMIN HMGB1 IS A CENTRAL MEDIATOR OF SENESCENT PHENOTYPES}

Davalos AR, Kawahara M, Malhotra GK, Schaum N, Huang J, Ved U, Beausejour CM, Coppe JP, Rodier F, Campisi J Lawrence Berkeley National Laboratory/Buck Institute for Research on Aging

Cellular senescence irreversibly arrests proliferation in response to potentially oncogenic stress. Senescent cells also secrete inflammatory cytokines such as IL-6, which promote age-associated inflammation and pathology. HMGB1 (High Mobility Group Box 1) modulates gene expression in the nucleus, but certain immune cells secrete HMGB1 as an extracellular Alarmin to signal tissue damage. We show that nuclear HMGB1 relocalized to the extracellular milieu in senescent human and mouse cells in culture and in vivo. In contrast to cytokine secretion, HMGB1 redistribution required the p53 tumor suppressor, but not its activator ATM. Moreover, altered HMGB1 expression induced a p53-dependent senescent growth arrest. Senescent fibroblasts secreted oxidized HMGB1, which stimulated cytokine secretion through TLR-4 signaling. HMGB1 depletion, HMGB1 blocking 
antibody or TLR-4 inhibition attenuated senescence-associated IL-6 secretion, and exogenous HMGB1 stimulated NF- $\kappa$ B activity and restored IL-6 secretion to HMGB1-depleted cells. Our findings identify senescence as a novel biological setting in which HMGB1 functions and link HMGB1 redistribution to p53 activity and senescence-associated inflammation.

111

\section{THE IN VITRO SENESCENCE PHENOTYPE OF MESENCHYMAL STROMAL CELLS AND POTENTIAL RAMIFICATIONS FOR INNATE IMMUNE FUNCTION}

Curran S, Campisi J

Buck Institute for Research on Aging

Mesenchymal stromal cells (MSCs) are multipotent adult stem cells that are found in specialized tissues, such as bone marrow, adipose tissue, and the umbilical cord; as well as systemically as pericytes or in the peripheral blood. MSCs are involved in multiple important processes, such as wound healing and tissue regeneration, but are of special interest for their capacity to suppress both the innate and acquired immune systems.

Cellular senescence, a tumor suppressor mechanism that manifests as a state of irreversible growth arrest and an augmented secretory profile, has been observed in many cells lines and tissues, including MSCs. While cellular senescence is well characterized in differentiated fibroblastic and epithelial cell lines, the senescence phenotype of MSCs is poorly understood, especially in the context of their immunosuppressive capacity.

Here, I describe the x-irradiation induced senescence phenotype of MSCs with a variety of canonical senescence markers: senescence associated $\beta$-galactosidase, DNA damage foci, the presence and activity of cell cycle inhibitors, and the senescence associated secretory phenotype. Additionally, I present preliminary data suggesting that the induction of cellular senescence can potentiate the immunosuppressive function of MSCs. These data lead to the hypotheses that MSCs may be implicated in the localized decline of immune system surveillance as well as systemic, age-related immune system senescence.

112

\section{WHY ORTHODOX MEDICINE MUST CHANGE - THE NEED FOR PREVENTATIVE/REGENERATIVE MEDICINE}

Micans $P$

International Antiaging Systems

Most people realize that the world's population is at the same time expanding and getting older and that these issues present many challenges. This lecture aims to highlight the facts with a view to the cost of 'orthodox' medicine- asking the question; should medicine as it exists today be changed from its present criteria?

Many healthcare professionals in the preventative medicine field know that there are many 'alternative/ unapproved' solutions, but perhaps lack the details of why it is actually necessary in the first place. This lecture will provide the interested attendee with the facts as issued by the US, UK and WHO authorities.

It will become clear that 'orthodox' medicine- as it exists today, cannot continue as-is and that a different path will need to be taken; therefore positioning preventative and regenerative medicine as the most likely and sensible alternative.

In Phil's indomitable style, he attempts, to challenge who, what and why will be at the fore, what the facts of the aging populous are and the concept of the optimal health pyramid. 


\section{Abstract Author Index by abstract number}

A

Accardi G, 1, 16, 104

Akman K, 39

Albanna M, 45

Almeida-Porada G, 2

Alvarez P, 69

Anderson RM, 3, 4

Antrobus R, 62

Appleby J, 5

Arancio W, 6

Aronov S, 100

Ayyadevara S, 89

Azzarello DM, 11

\section{B}

Balasubramaniam M, 89

Balietti M, 17

Balistreri CR, 7, 14

Balva OV, 90

Barker RW, 8

Bartlett H, 101

Beausejour CM, 110

Beliakoff $\mathrm{G}, 9$

Benedetto F, 7

Bentley B, 13

Berrett MG, 18

Bertuccelli G, 43, 67

Blinder V, 75

Boominathan A, 74

Boone L, 59

Boura J, 2

Braiman A, 100

Brown K, 19

Budovsky A, 10, 70, 99, 100, 107

Buffa S, 11

Buiucli S, 88

Bulati M, 11

Busse K, 45

C

Cabeca A, 67

Cai D, 12

Calimport SRG, 13

Caliò A, 107

Camarda C, 11

Campisi J, 79, 110, 111

Candore G, 1, 7, 11, 14, 16, 104

Cao YC, 15

Caruso C, 1, 7, 11, 14, 16, 104

Casoli T, 17

Catanzaro R, 43, 47, 67, 68

Cawthon RM, 18

Celep G, 47, 67, 68
Chen D, 19

Chen-Tsai RY, 20

Chilosi M, 107

Chin G, 21

Church G, 22

Ciccarelli F, 23, 37

Coeurnelle D, 24

Colonna-Romano G, 7, 11, 104

Cook B, 82

Coppe JP, 110

Corder EH, 25, 26

Craig T, 29

Crampton A, 74

Curran S, 111

\section{D}

d'Alessio P, 27

Davalos AR, 110

Davenport MP, 94

Debonneuil E, 90

de Cabo R, 44

de Magalhães JP, 28, 29, 99

De Martinis M, 23, 37

Dennis J, 93

DeWard A, 59

Di Bona D, 16

Di Maggio FM, 14

Diaz F, 40

Dominko T, 30

Duman O, 10

E

Emancipator SN, 76

Emanuele F, 1

\section{F}

Fabiny L, 82

Fattoretti $\mathrm{P}, 17$

Fontana L, 31

Forte GI, 7

Frackelton JP, 76

Fraifeld VE, 10, 61, 70, 99, 100, 107

Franklin RJM, 32

Furber JD, 33, 34

G

Ganapathi S, 39

Garcia Guerrero O, 35

Gaspar J, 36

Gervasi F, 11

Ginaldi L, 23, 37

Giordano C, 6

Giorgetti B, 17
Giuli C, 17

Gneteeva T, 88

Gobshtis N, 100

Goldin E, 9

Goligorsky MS, 38

Gravina S, 39

Grechko N, 105

Greenberg J, 81

Gygi SP, 62

\section{H}

Hall W, 101

Halvorsen D, 91

Hara M, 75

He F, 68

He M, 19

Hébert JM, 40

Herbig E, 85

Hockemeyer D, 41

Hoppo T, 59

$\mathrm{Hu} \mathrm{MH}, 42$

Huang J, 110

Huang ZB, 42, 64

Hunt T, 91

I

Illuzzi N, 43, 47, 67, 68

Ingram DK, 4, 44

Italia A, 43, 67

J

Jackson JD, 45

Jones T, 103

Ju YM, 45

Ju Z, 46

K

Kang W, 40

Kantah MK, 47

Kawahara M, 110

Kerber RA, 18

Khaltourina, 51

Khalyavkin AV, 48, 49, 50

Khan SM, 93

Kikkawa K, 52

Kim N, 45

Kirkwood TBL, 55

Kletetschka G, 53

Klimova L, 53

Kobayashi R, 47

Komori J, 59

Kope M, 54

Kowald A, 55 
Krøll J, 56

Krutko VN, 48

Kuhn R, 57

Kulaga A, 106

Kurdyavtseva AV, 72

Kurtak K, 58

\section{L}

Lagasse E, 59

Larrick JW, 60

Lehmann G, 61

Lehner PJ, 62

Lentz MR, 63

Li G, 94

Li HF, 42, 64

Liang M, 64

Licastro F, 1

Lio $\mathrm{D}, 7,11,14$

Litovchenko M, 109

Liu Y, 19

Lonsdale D, 76

Lorenzetti A, 67

\section{M}

Ma FL, 42, 64

Maden M, 65

Madeo F, 66

Malhotra GK, 110

Mao XL, 64

Maor H, 10

Maresi E, 14

Marotta F, 43, 47, 67, 68

Martorana A, 11

Mathieu J, 69

Mattison JA, 44

Mazuz K, 70

McKeehan N, 40

Mendelsohn A, 60

Micans P, 112

Milazzo M, 43, 47, 67, 68

Mitteldorf J, 71

Mkrtchyan G, 88

Mohania D, 43

Mokhtari S, 2

Moody K, 82, 91

Moskalev AA, 72

Muradian KhK, 61

\section{$\mathbf{N}$}

Nagpal R, 43

Naito $Y, 43$

Nawaz MS, 98

Needham SL, 73

Nikolich-Zugich, 94

Nishiyama Y, 75

\section{O}

Obrenovich ME, 76

O'Brien E, 18

O'Connor M, 74

Oishi R, 52

Okada H, 52
O'Nuallain B, 75

Onyango I, 93

\section{$\mathbf{P}$}

Park E, 77

Parnia S, 78

Paul S, 75

Paulo F, 59

Perrott KM, 79

Phay M, 75

Pisano C, 14

Pishel IM, 80, 90

Pizzolanti G, 6

Planque SA, 75

Plyusnina EN, 72

Poole E, 62

Porada C, 2

Porat Z, 100

Portell RF, 93

Pyszczynski T, 81

Q

Qiu J, 20

Qiu X, 19

R

Rao A, 59

Rastmanesh R, 68

Rebo J, 82

Rider TH, 83

Rodier F, 110

Roth GS, 44

Russell A, 84

Ruvolo G, 7, 14

Ryu SW, 45

S

Sapienza C, 43, 47, 67, 68

Schaum N, 110

Schloendorn J, 82

Scholz M, 85

Scola L, 14

Sedivy JM, 86

Shannack M, 87

Sharma V, 60

Shin J, 19

Shindyapina AV, 88

Shmookler-Reis R, 89

Shteinberg A, 10

Shytikov DW, 90

Silva $\mathrm{H}, 91$

Sinclair JH, 62

Sitnicki I, 92

Smelick C, 29

Smigrodzki R, 93

Smith DL, 62

Smithey MJ, 94

Soker S, 45

Soland M, 2

Solazzi M, 17

Solimene U, 68

Song J, 64

Spiegel DA, 95

Spinelli F, 7
Srivastava N, 68

Stambler I, 96

Stolzing A, 97

Sukhera UA, 98

$\mathrm{T}$

Tacutu R, 99, 107

Takadanohara H, 47

Talbot S, 62

Tan S, 62

Taranukha D, 100

Tomella C, 43, 47, 67

Tresch A, 39

U

Underwood M, 101

V

van Deursen JM, 102

Vanhoozer S, 74

van Sickle S, 103

Vasto S, 1, 104

Ved U, 110

Vengalam J, 74

Venturi V, 94

Verga $S, 1$

Vijg J, 39

Virruso C, 1, 16, 104

Voronina T, 105

W

Wages J, 60

Wang H, 60

Wang J, 60

Watanabe K, 75

Webb T, 82

Weekes MP, 62

Wei $X, 60$

Wolfson M, 10

Wright SC, 60

Wuttke D, 106

X

Xiang YX, 42

Xiao LY, 42

Xie S, 19

Xu M, 85

Y

Yaduvanshi SK, 68

Yanai H, 10, 107

Yang F, 64

Yogalingam G, 9

Yoo JJ, 45

Youle RJ, 108

Z

Zerbinati N, 43, 67

Zhang J, 42

Zhang T, 20

Zhavoronkov A, 88, 109

Zheng Q, 20

Zila V, 53 